\title{
Sulfenylation of arenes with ethyl arylsulfinates in water
}

Yueting Wei, ${ }^{\mathrm{a}}$ Jing He, ${ }^{\mathrm{a}}$ Yali Liu, ${ }^{\mathrm{a}}$ Liang $\mathrm{Xu},{ }^{\mathrm{a}}$ Luigi Vaccaro, ${ }^{\mathrm{c}}$ Ping Liu, ${ }^{\text {a }}$ and Yanlong $\mathrm{Gu}^{* \mathrm{~b}}$

a School of Chemistry and Chemical Engineering, the Key Laboratory for Green Processing of Chemical Engineering of Xinjiang Bingtuan, Shihezi University, Shihezi City, 832004, China.

${ }^{\mathrm{b}}$ Key Laboratory of Material Chemistry for Energy Conversion and Storage, Ministry of Education, Hubei Key Laboratory of Material Chemistry and Service Failure, School of Chemistry and Chemical Engineering, Huazhong University of Science \& Technology, 1037 Luoyu road, Hongshan District, Wuhan 430074, China.

c Laboratory of Green S.O.C. -Dipartimento di Chimica, biologia e Biotecnologie, Università degli Studi di Perugia, Via Elce di Sotto 8, 06123 -Perugia, Italy.

E-mail: liuping1979112@aliyun.com (P. Liu); klgyl@hust.edu.cn (Y. Gu). Tel.: +86 0993 2057213; Fax: +8609932057270.

${ }^{1} \mathrm{H}$ NMR, ${ }^{13} \mathrm{C}$ NMR and ${ }^{19} \mathrm{~F}$ NMR Spectra of Products 2 


\section{${ }^{1} \mathrm{H}$ NMR, ${ }^{13} \mathrm{C}$ NMR and ${ }^{19}$ F NMR Spectra of Products}

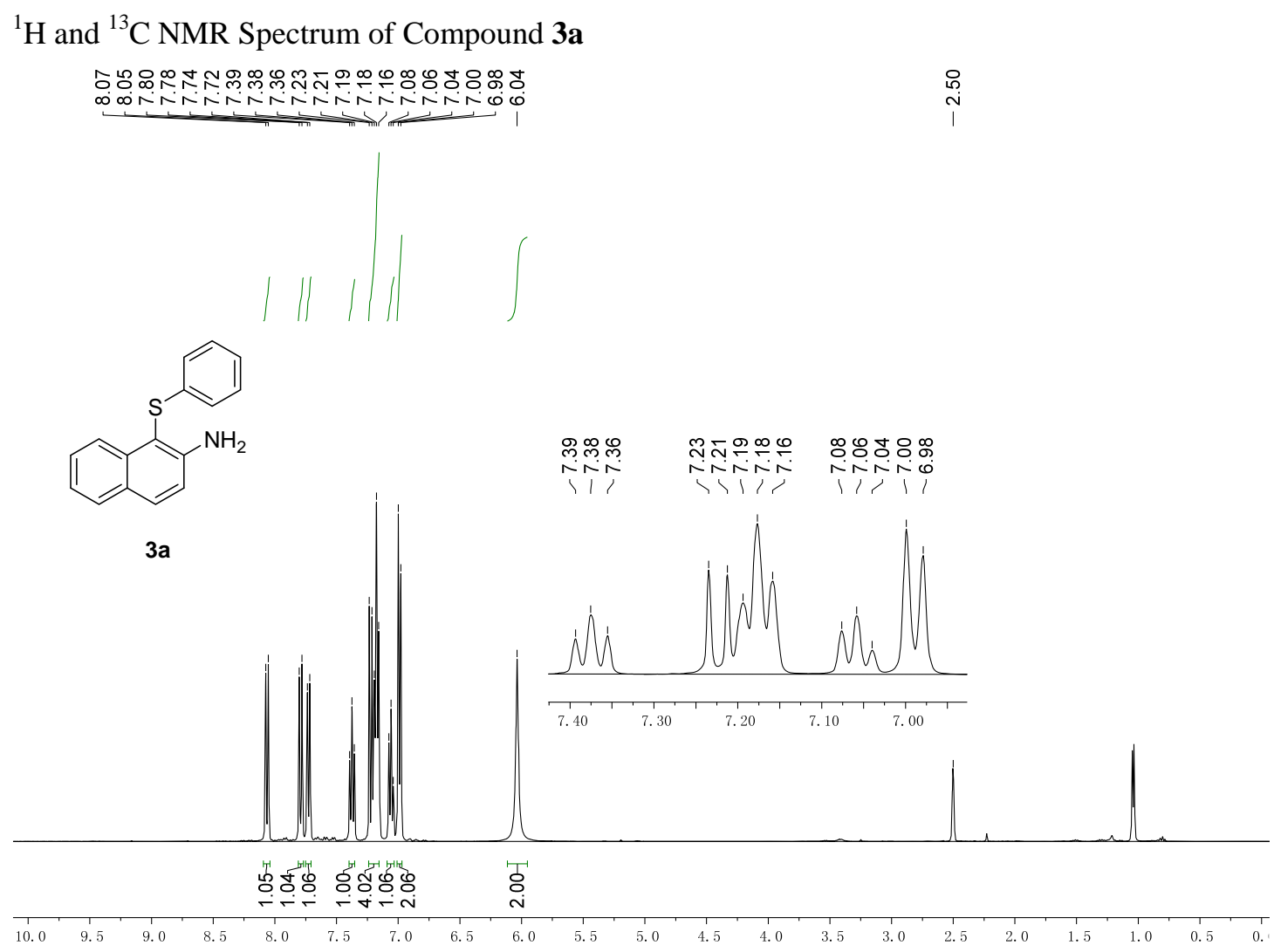

Figure S1

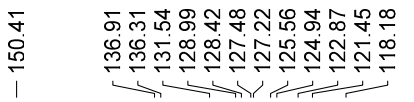

용

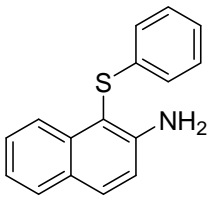

$3 a$

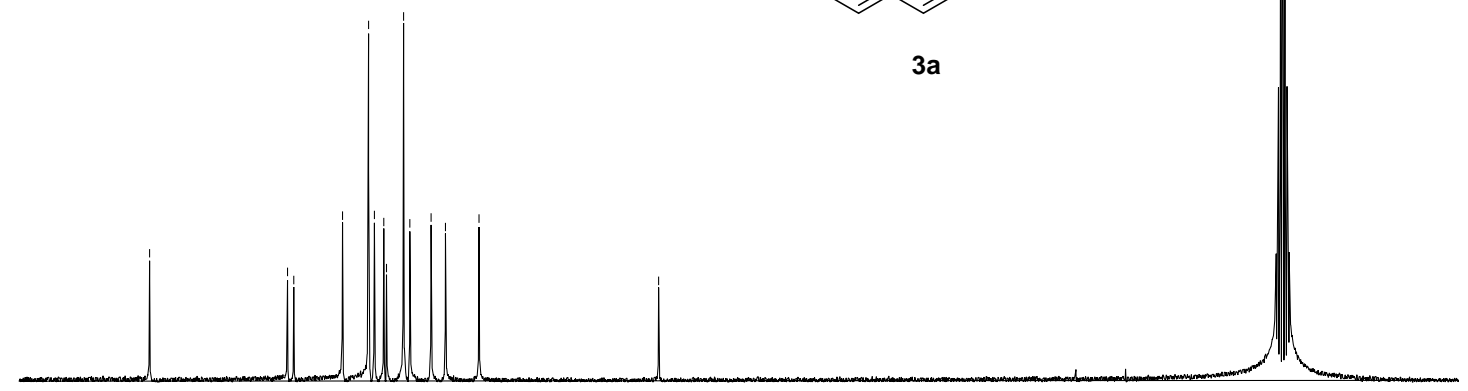

$155 \quad 145 \quad 135$ $95 \quad 90$

Figure S2 
${ }^{1} \mathrm{H}$ and ${ }^{13} \mathrm{C}$ NMR Spectrum of Compound $3 \mathbf{b}$

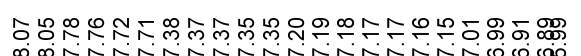

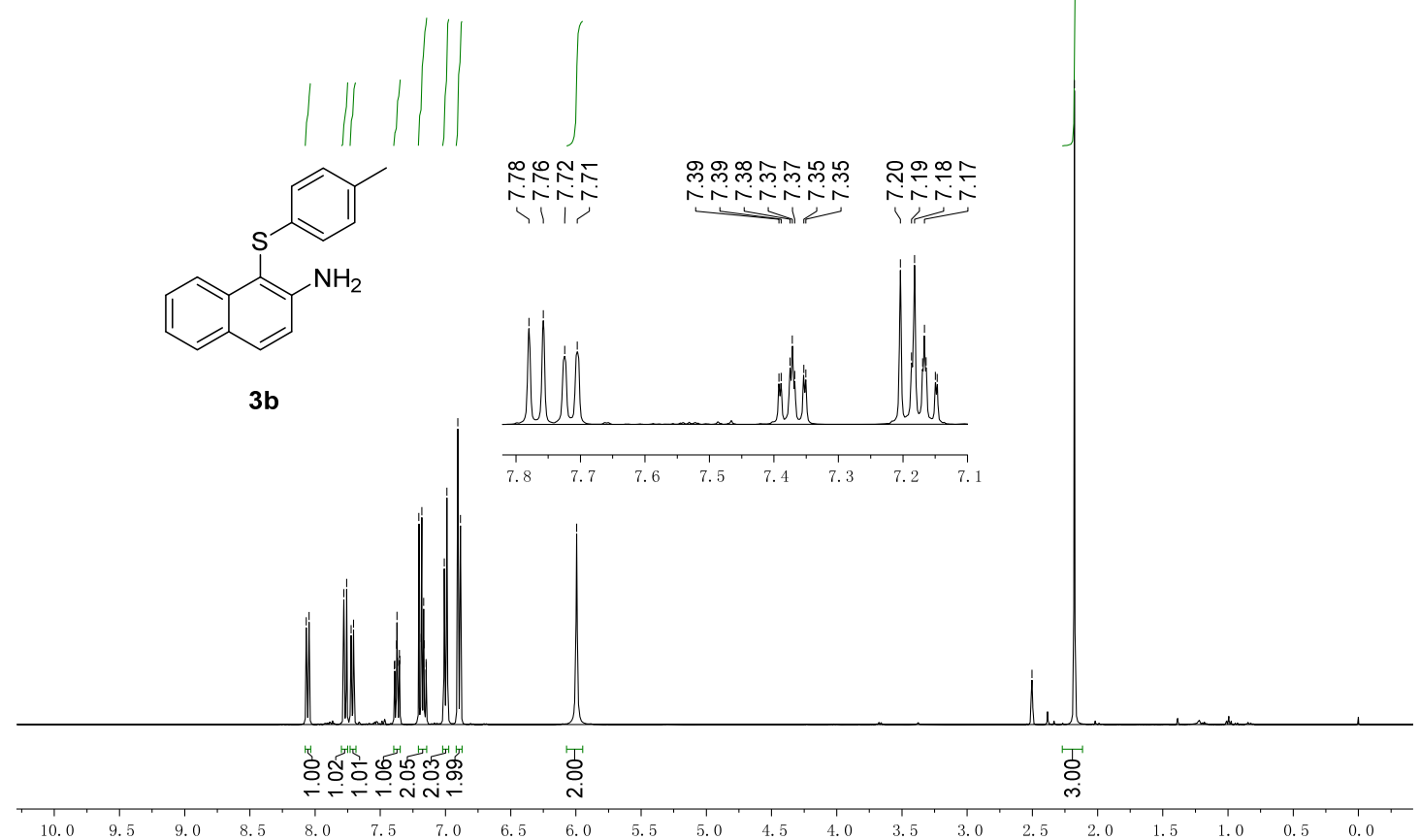

Figure S3
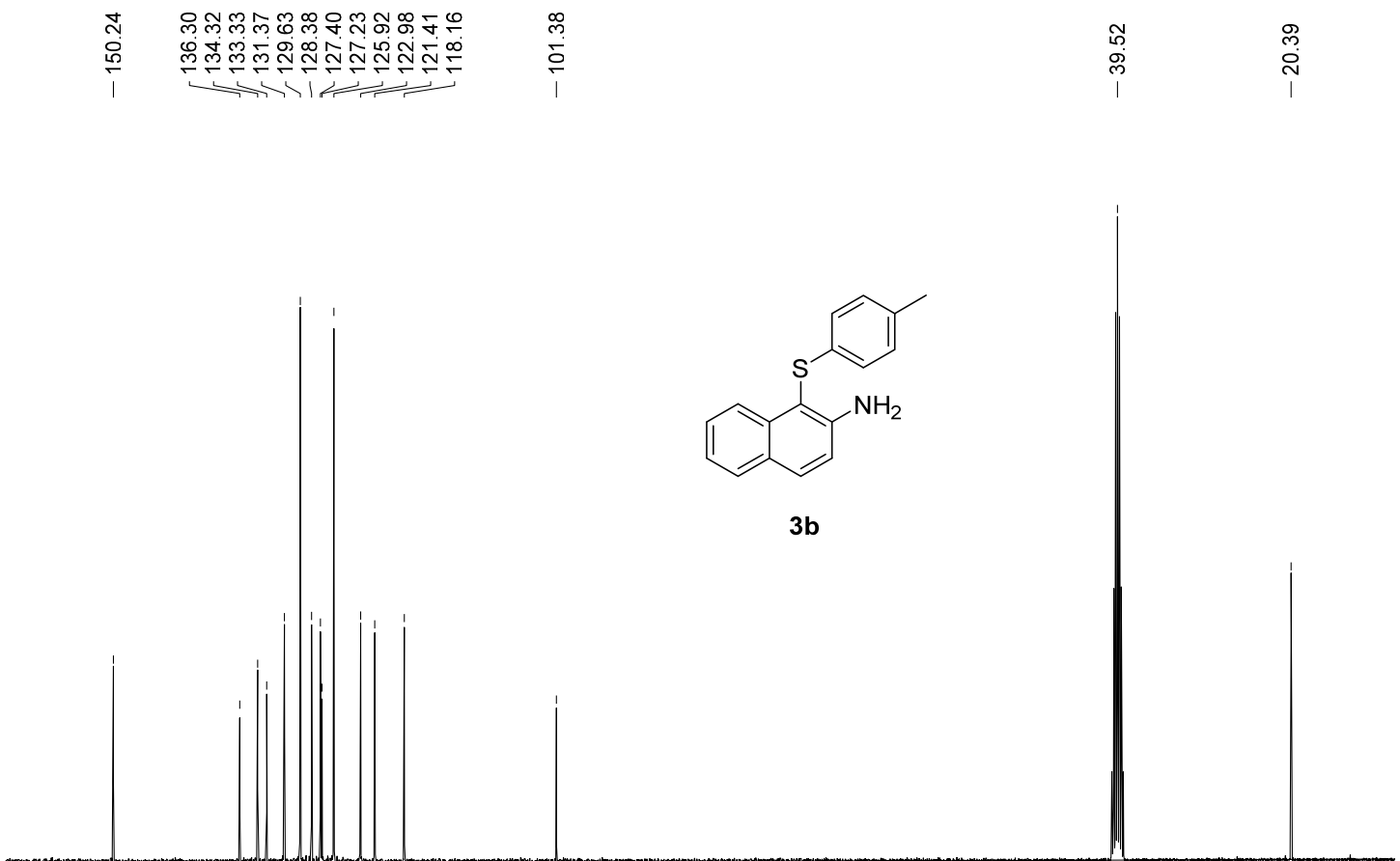

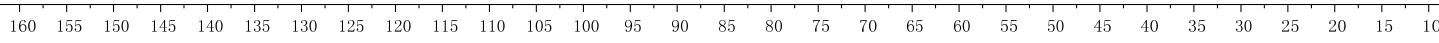

Figure S4 
${ }^{1} \mathrm{H}$ and ${ }^{3} \mathrm{C}$ NMR Spectrum of Compound 3c

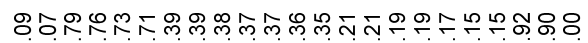

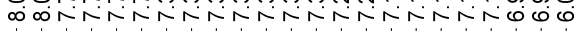

$\stackrel{\infty}{\stackrel{0}{i}} \stackrel{\stackrel{\infty}{\leftarrow}}{\Gamma}$

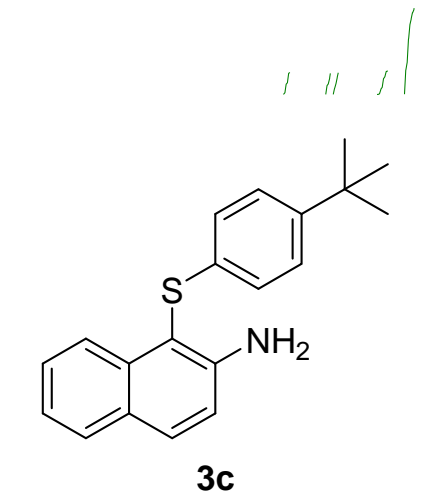

mলmm

nNiña nNiñNan
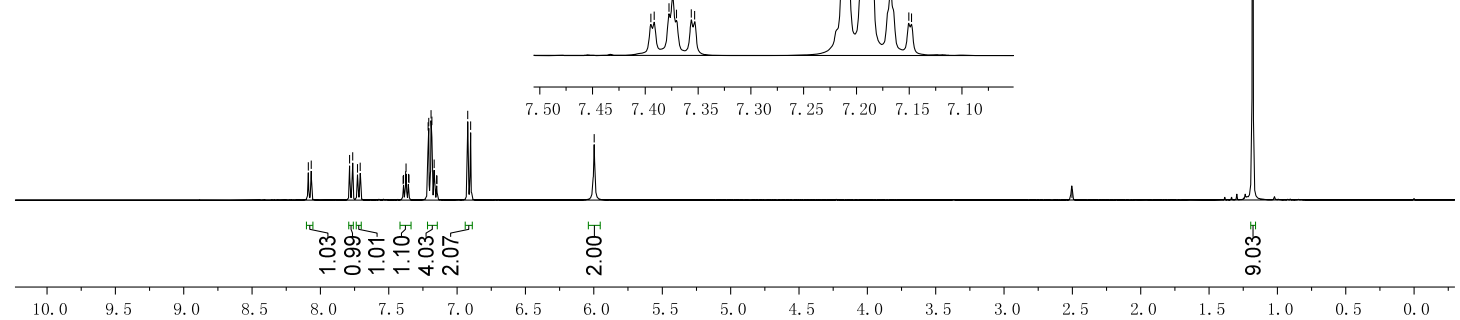

Figure S5

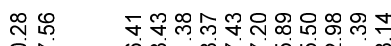

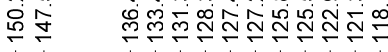

$\stackrel{\infty}{\frac{\infty}{5}}$

त̂.

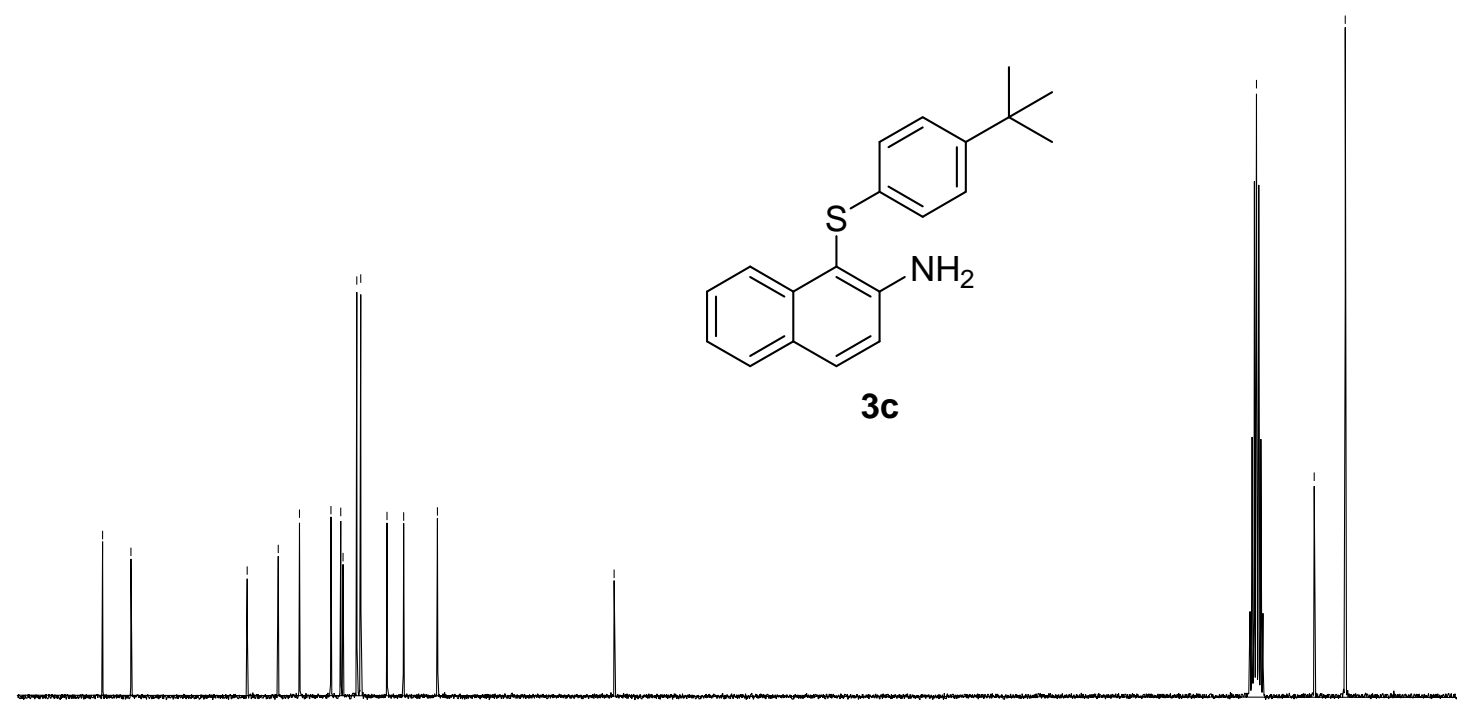

$\begin{array}{lllllllllllllllllllllllllll}155 & 150 & 145 & 140 & 135 & 130 & 125 & 120 & 115 & 110 & 105 & 100 & 95 & 90 & 85 & 80 & 75 & 70 & 65 & 60 & 55 & 50 & 45 & 40 & 35 & 30 & 25\end{array}$

Figure S6 
${ }^{1} \mathrm{H}$ and ${ }^{13} \mathrm{C}$ NMR Spectrum of Compound 3d

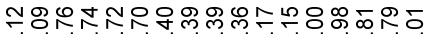

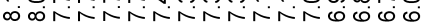

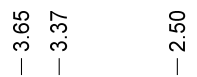
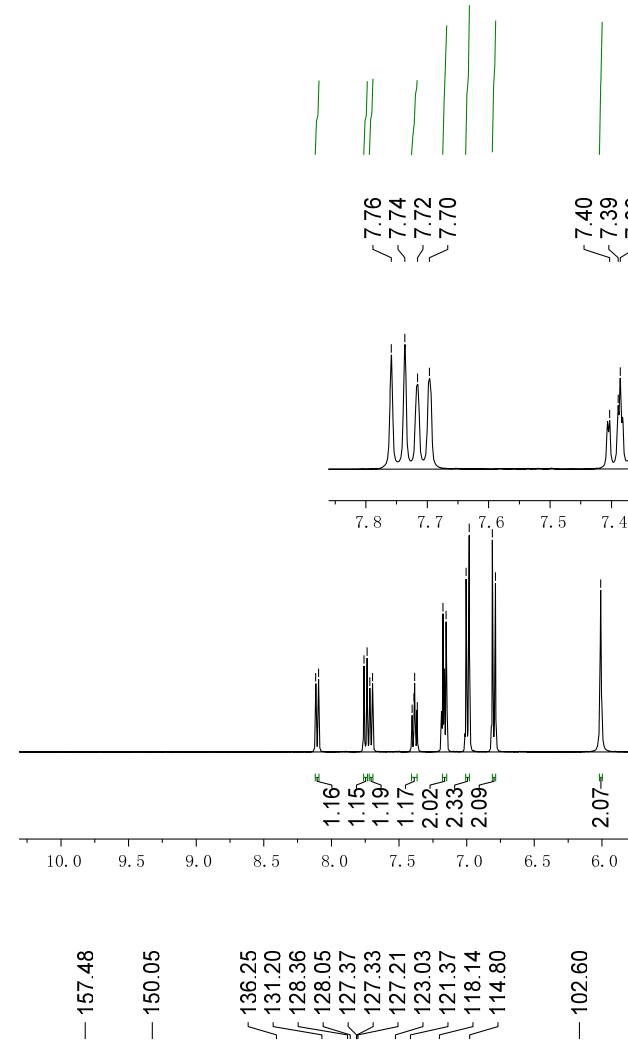

Figure S7

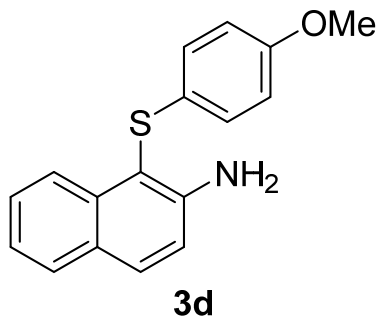<smiles>COc1ccc(Sc2c(N)ccc3ccccc23)cc1</smiles>

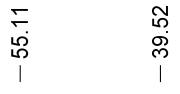
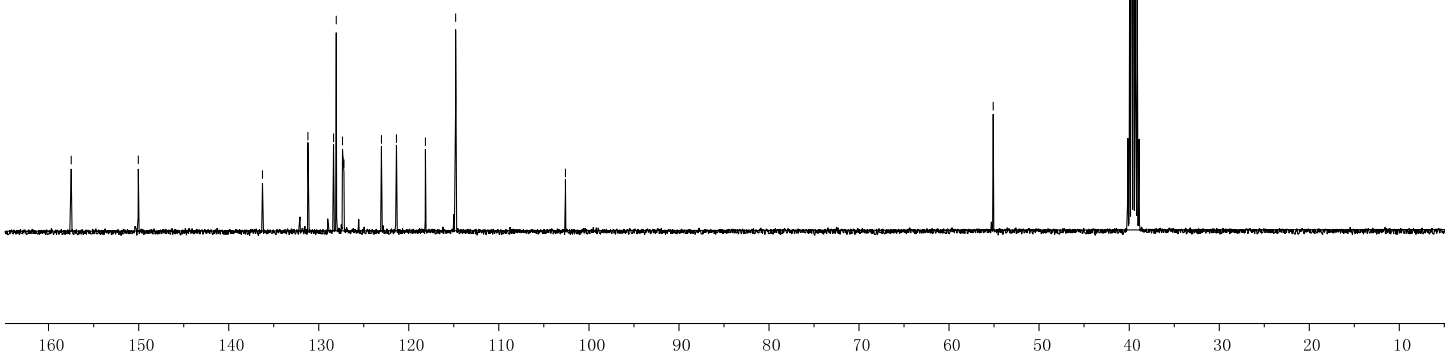

Figure S8 
${ }^{1} \mathrm{H}$ and ${ }^{13} \mathrm{C}$ NMR Spectrum of Compound $3 \mathbf{e}$

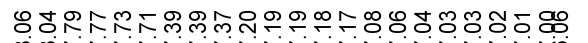

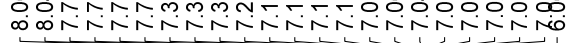

$\stackrel{\circ}{\text { i }}$
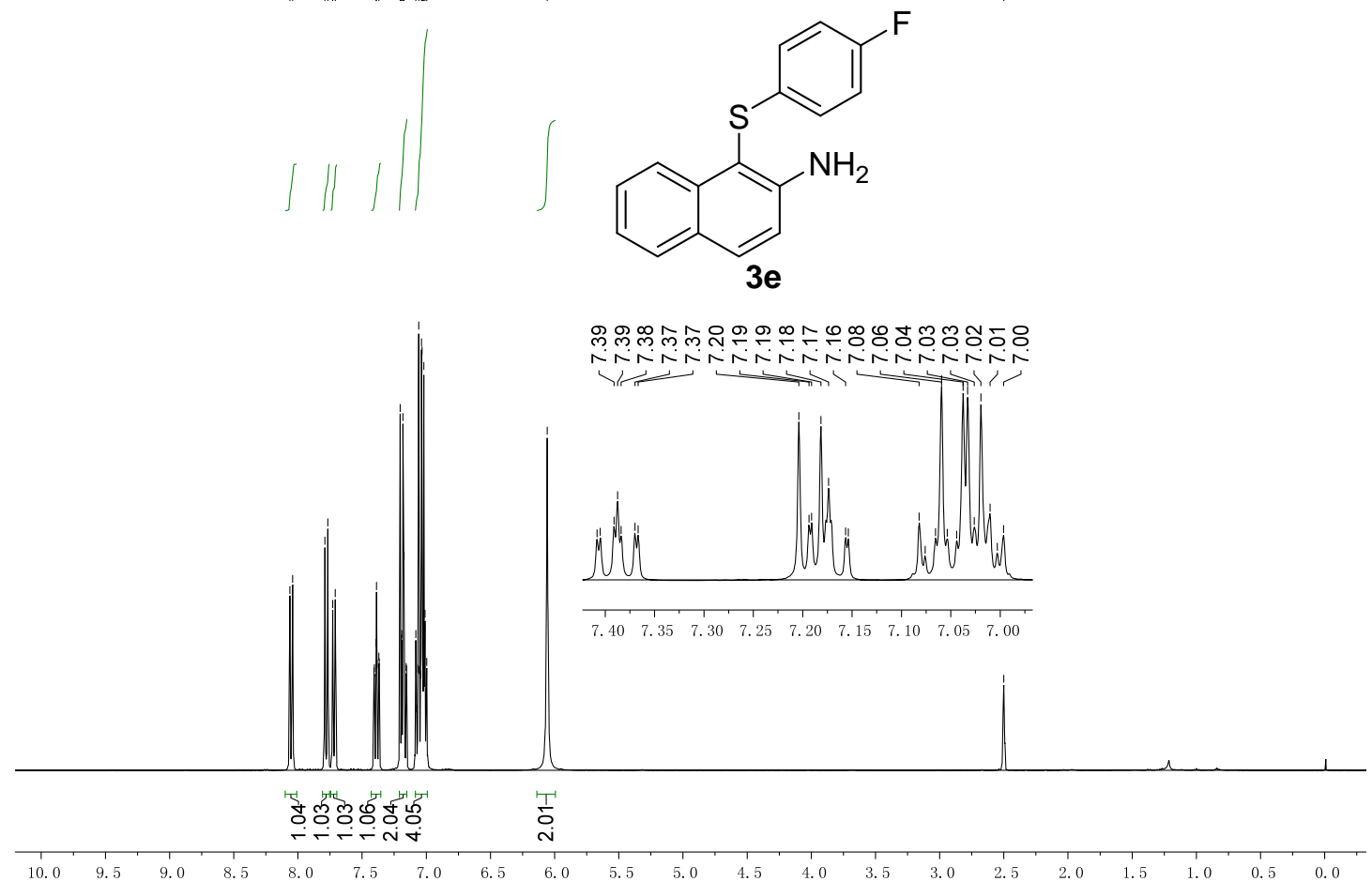

Figure S9

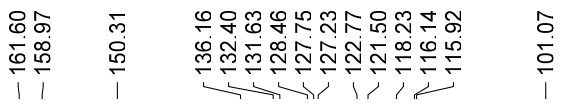<smiles>Nc1cccc2ccccc12</smiles>

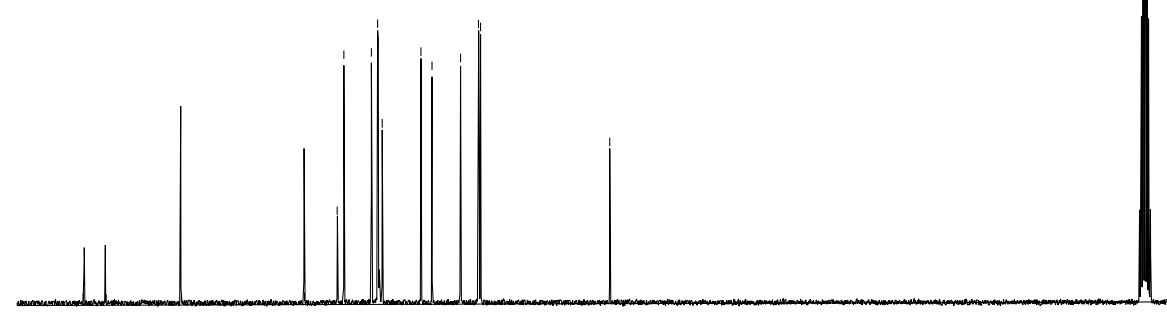

Figure S10 
${ }^{1} \mathrm{H}$ and ${ }^{13} \mathrm{C}$ NMR Spectrum of Compound $3 \mathbf{f}$

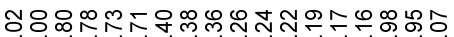

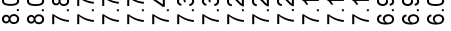
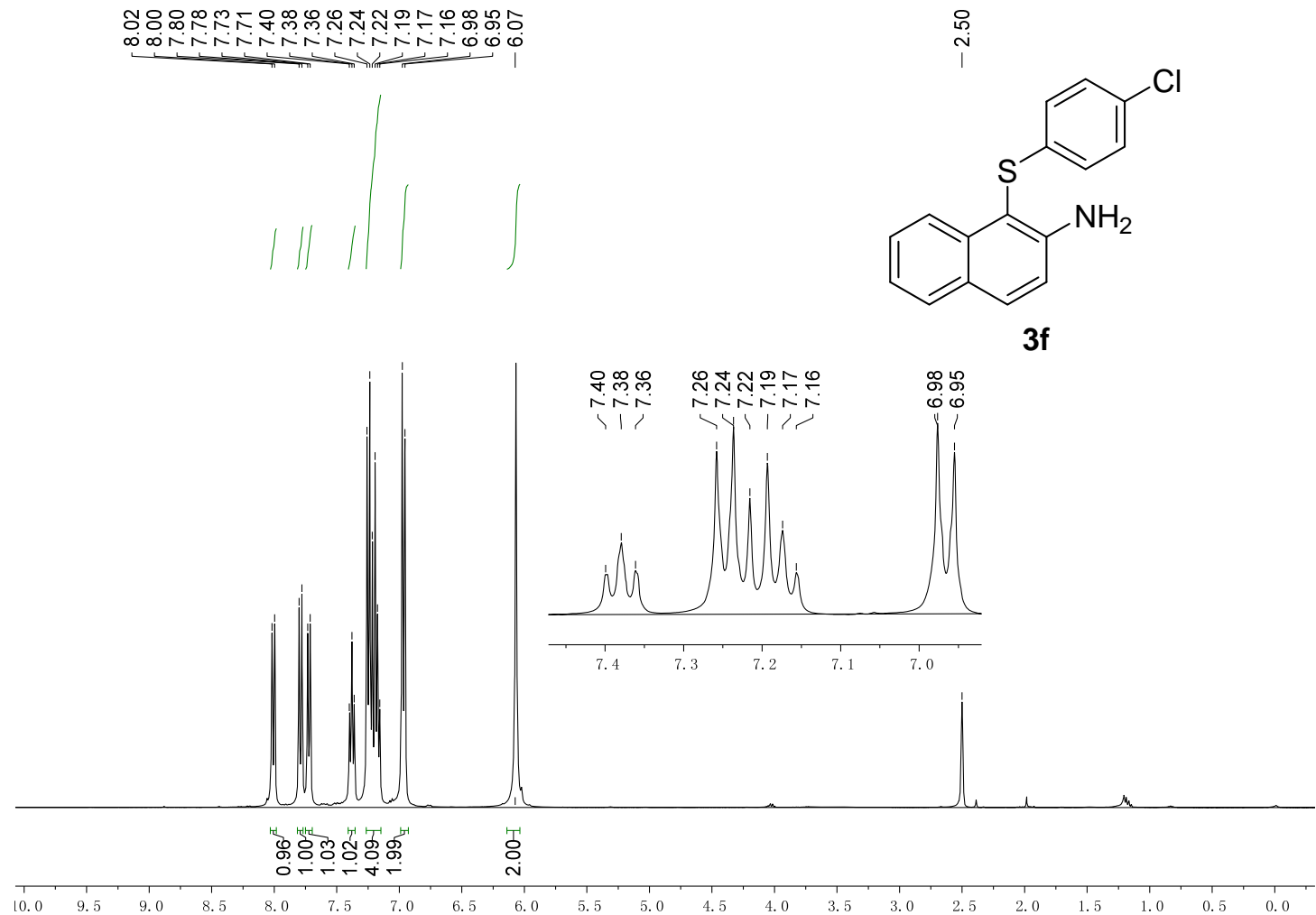

Figure S11

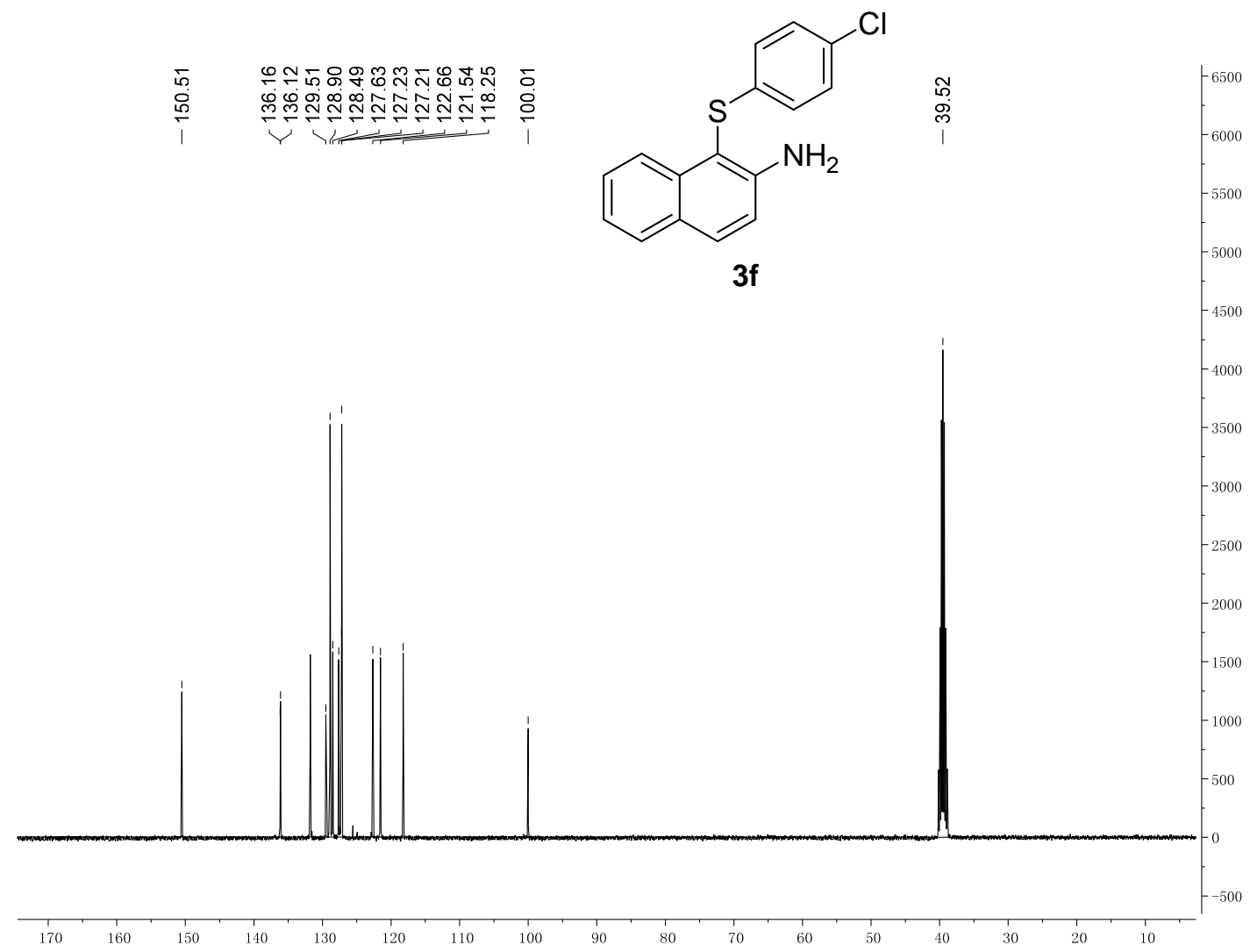

Figure S12 
${ }^{1} \mathrm{H}$ and ${ }^{13} \mathrm{C}$ NMR Spectrum of Compound $3 \mathbf{g}$

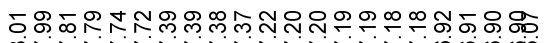

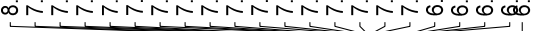

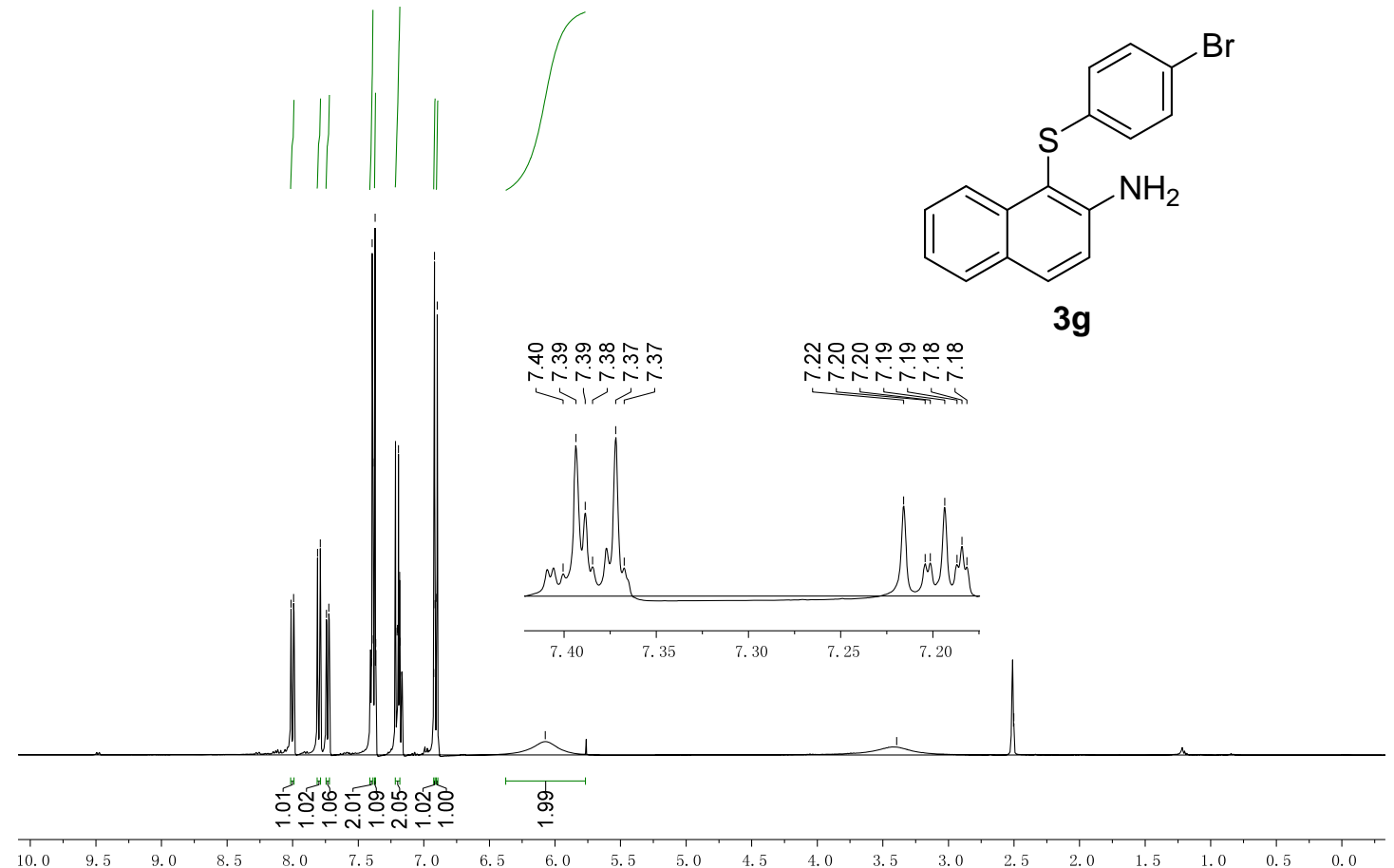

Figure S13

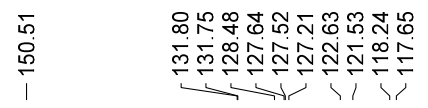

m<smiles>Nc1ccc2ccccc2c1Sc1ccc(Br)cc1</smiles>

$3 g$

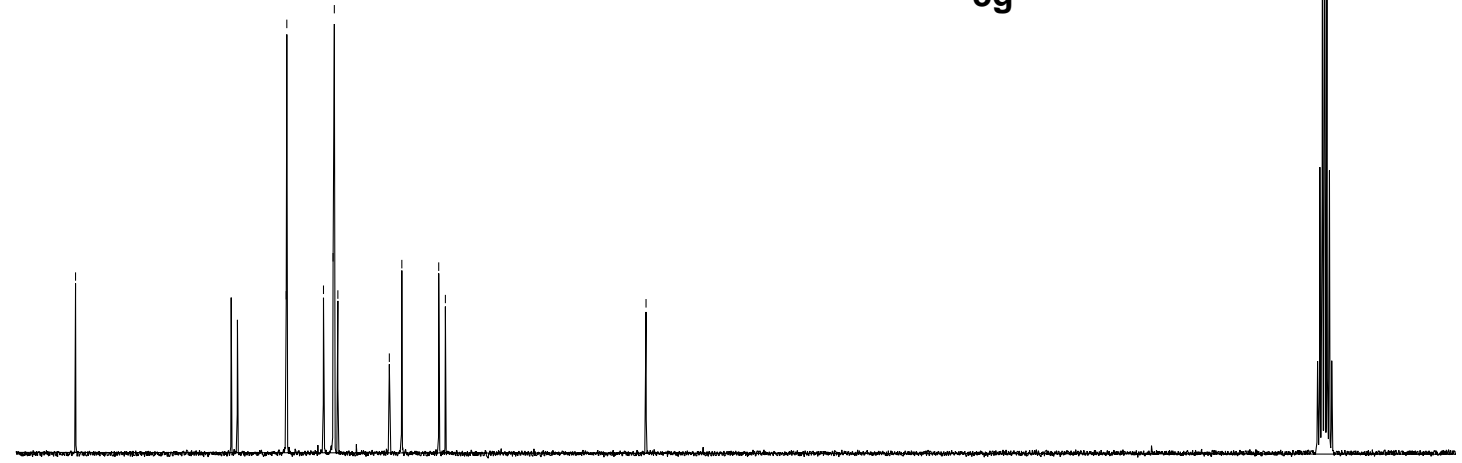

$\begin{array}{lllllllllllllllllllllllllllll}\mid & \mid \\ 155 & 150 & 145 & 140 & 135 & 130 & 125 & 120 & 115 & 110 & 105 & 100 & 95 & 90 & 85 & 80 & 75 & 70 & 65 & 60 & 55 & 50 & 45 & 40 & 35 & 30\end{array}$

Figure S14 
${ }^{1} \mathrm{H}$ and ${ }^{13} \mathrm{C}$ NMR Spectrum of Compound $3 \mathbf{h}$

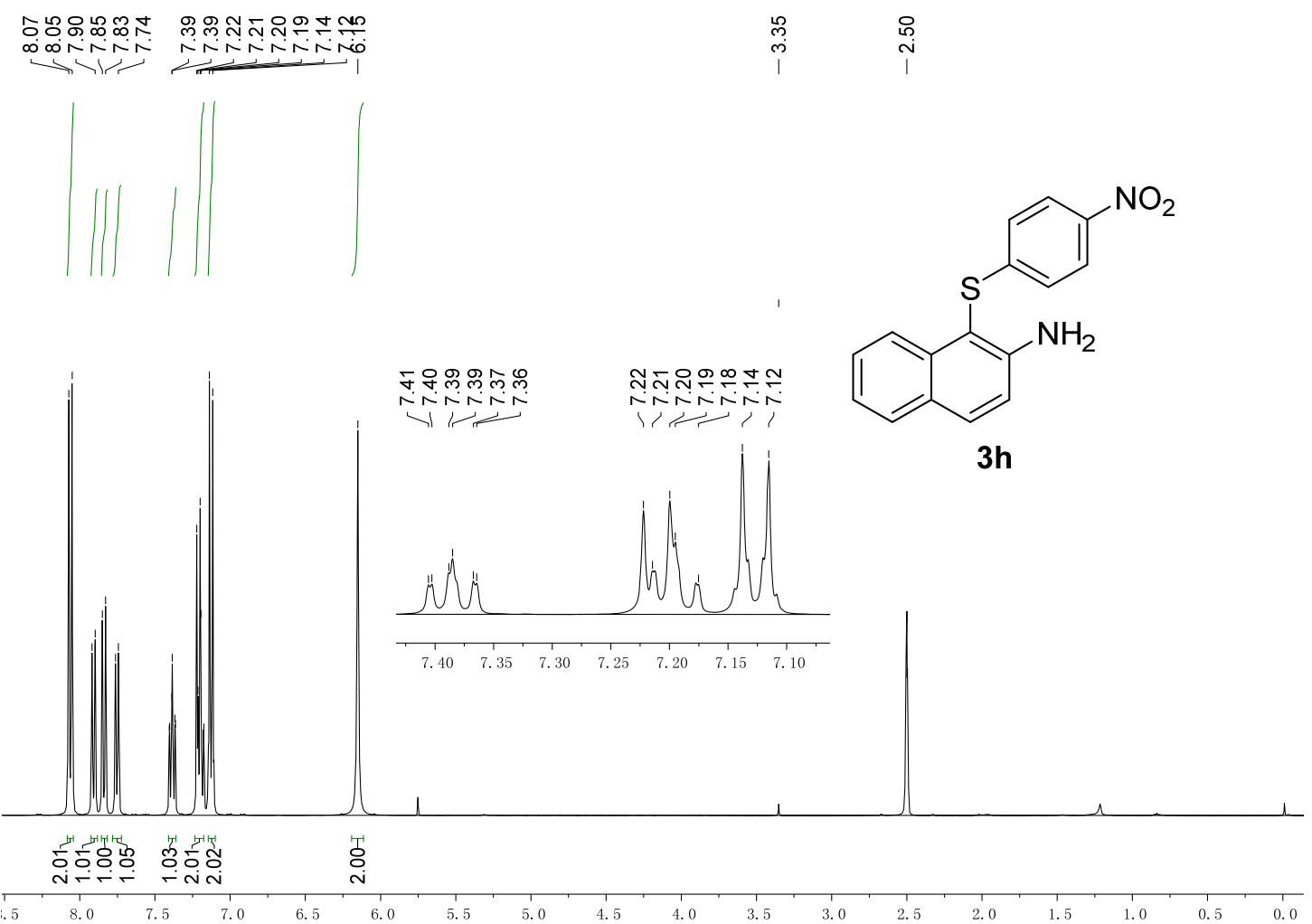

Figure S15

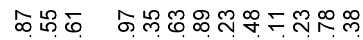

它守过

$1 \underbrace{-1}$

?

స్ำ<smiles>Nc1ccc2ccccc2c1Sc1ccc([N+](=O)[O-])cc1</smiles>

$3 \mathrm{~h}$

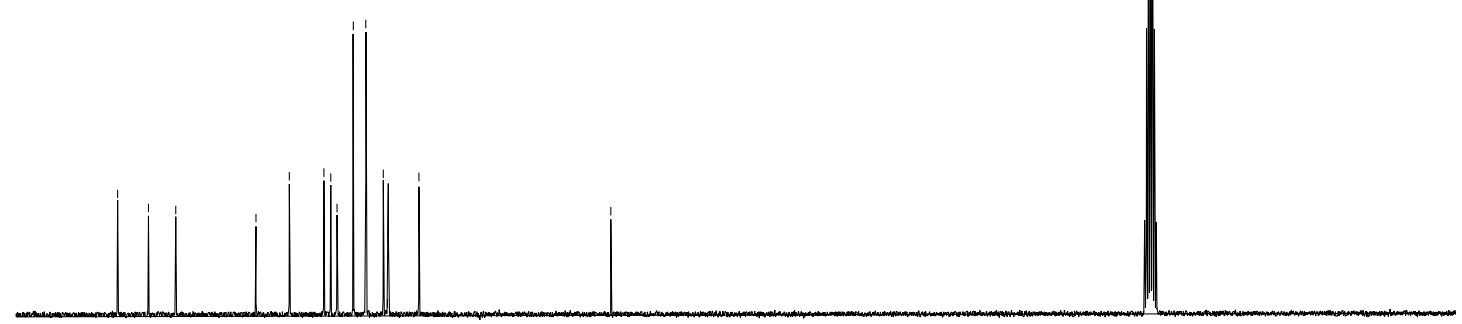

$\begin{array}{lllllllllllllllllllllllllllllllllll}160 & 155 & 150 & 145 & 140 & 135 & 130 & 125 & 120 & 115 & 110 & 105 & 100 & 95 & 90 & 85 & 80 & 75 & 70 & 65 & 60 & 55 & 50 & 45 & 40 & 35 & 30 & 25 & 20 & 15 & 10\end{array}$

Figure S16 
${ }^{1} \mathrm{H}$ and ${ }^{13} \mathrm{C}$ NMR Spectrum of Compound $3 \mathbf{i}$

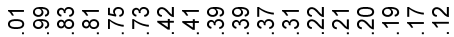

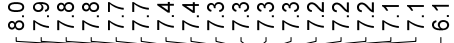
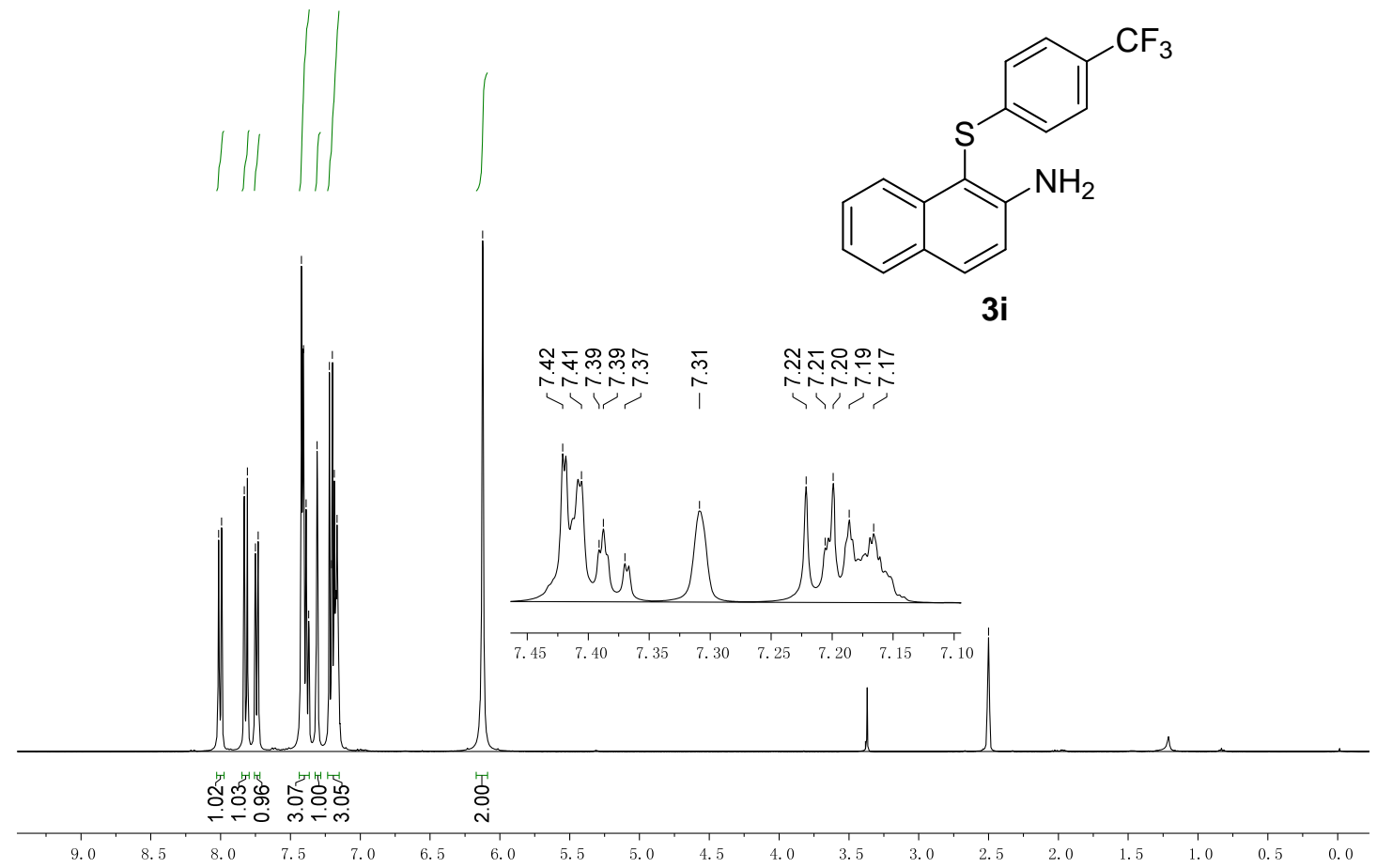

Figure S17

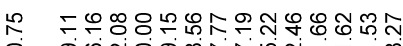

ำ

\%ั
o
।<smiles>Nc1ccc2ccccc2c1Sc1ccc(C(F)(F)F)cc1</smiles>

$3 \mathbf{i}$
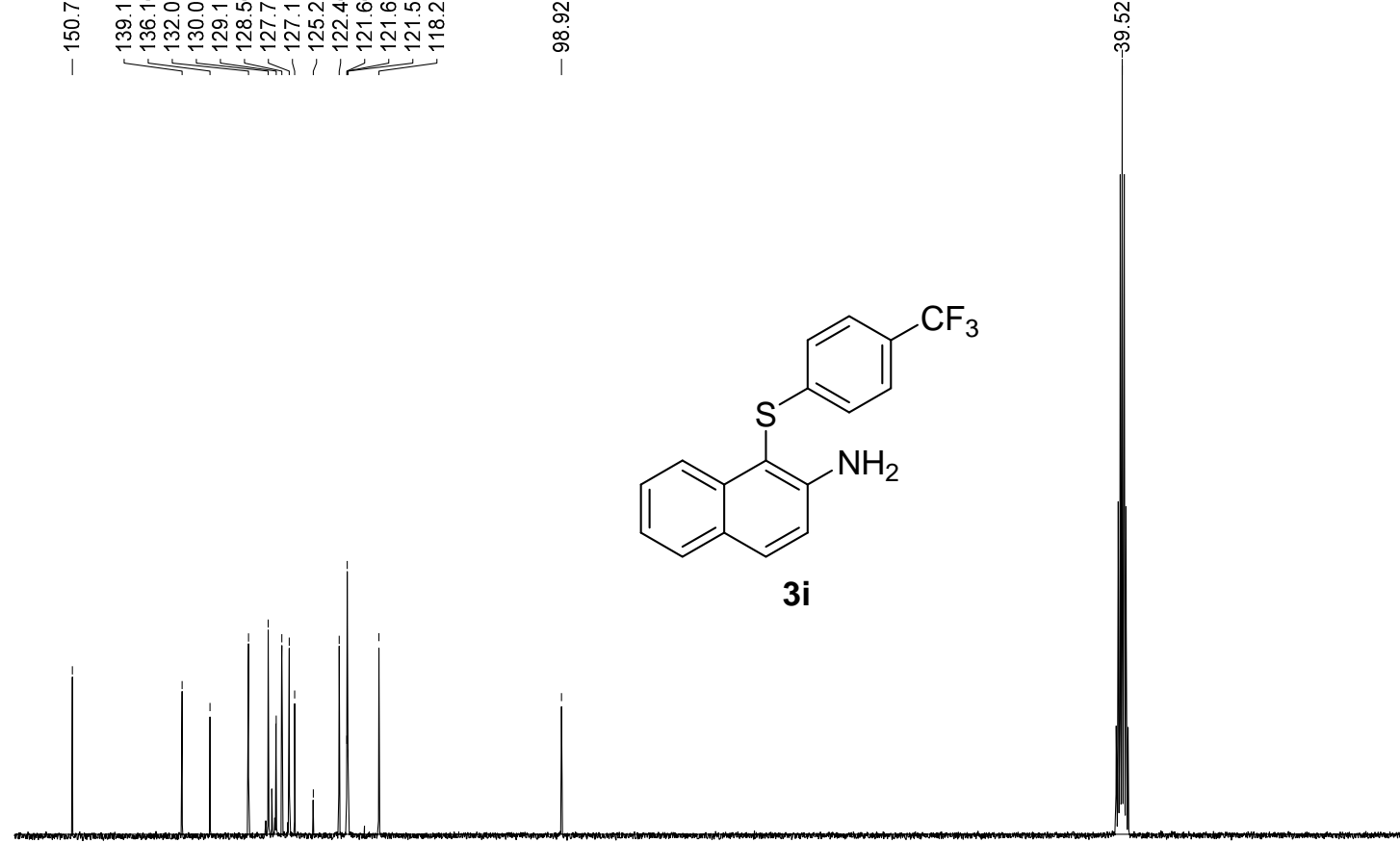

$\begin{array}{lllllllllllllllllllllllllllllllllllllllllllllll}155 & 150 & 145 & 140 & 135 & 130 & 125 & 120 & 115 & 110 & 105 & 100 & 95 & 90 & 85 & 80 & 75 & 70 & 65 & 60 & 55 & 50 & 45 & 40 & 35 & 30 & 25 & 20 & 15 & 1\end{array}$

Figure S18 
${ }^{1} \mathrm{H},{ }^{13} \mathrm{C}$ NMR and ${ }^{19} \mathrm{~F}$ NMR Spectrum of Compound $\mathbf{3 j}$

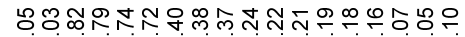

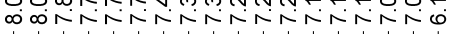
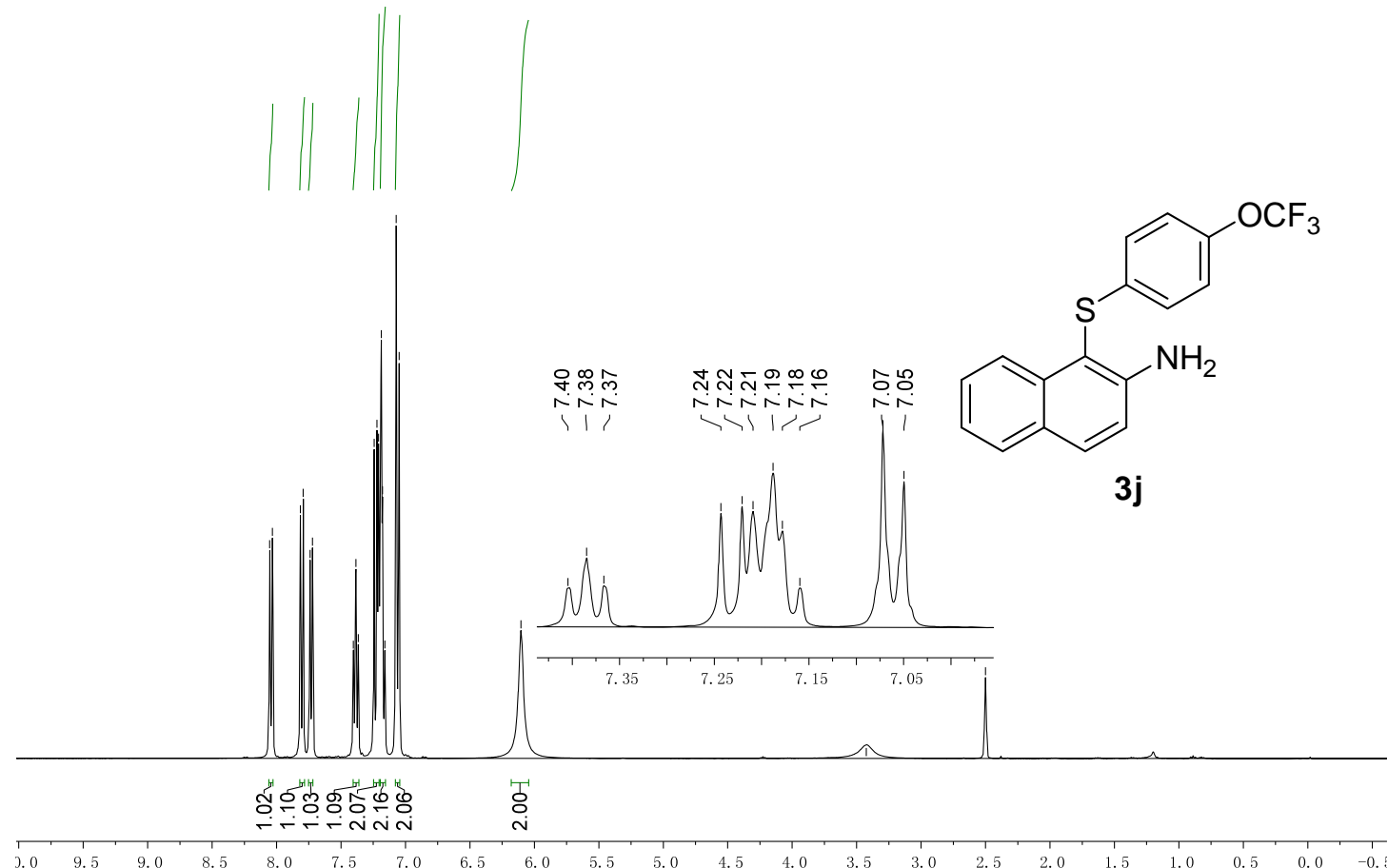

Figure S19

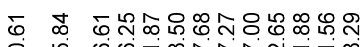

吕 品
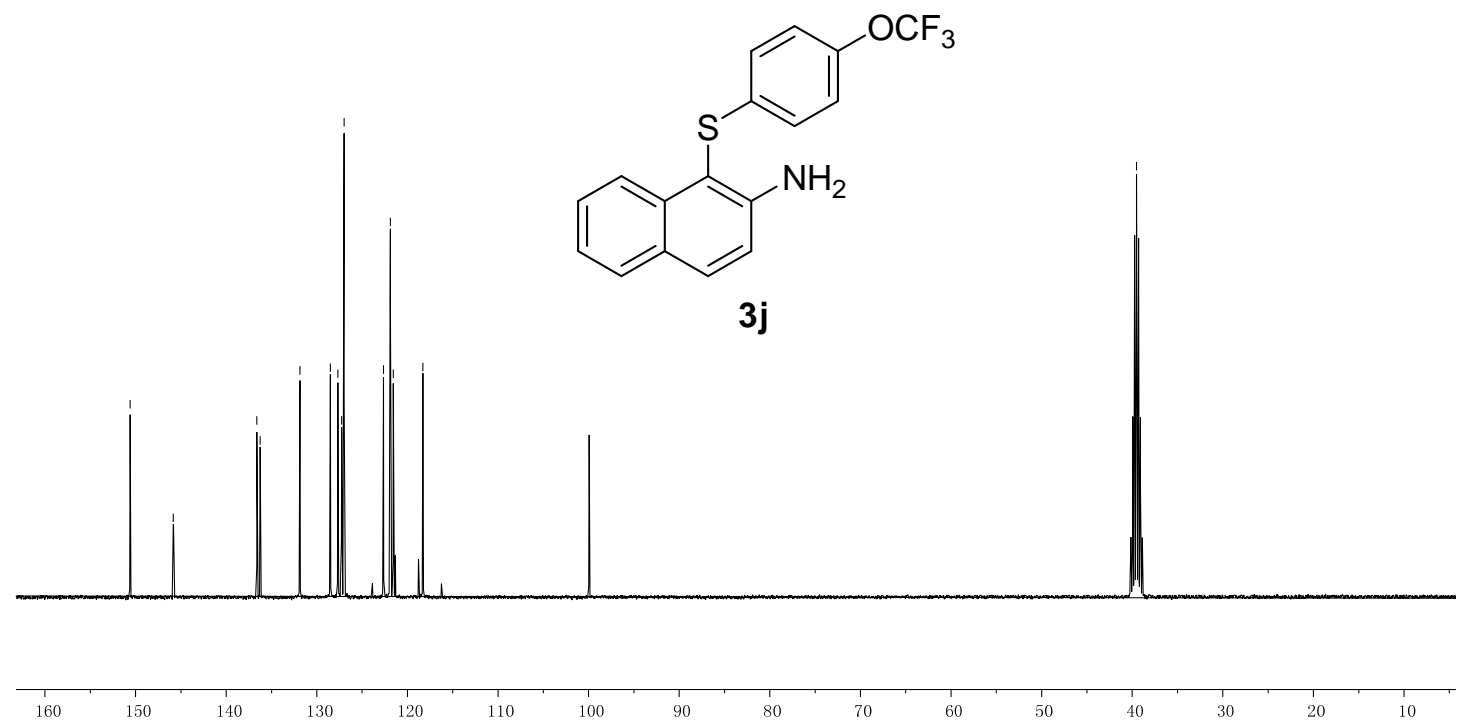

Figure S20 

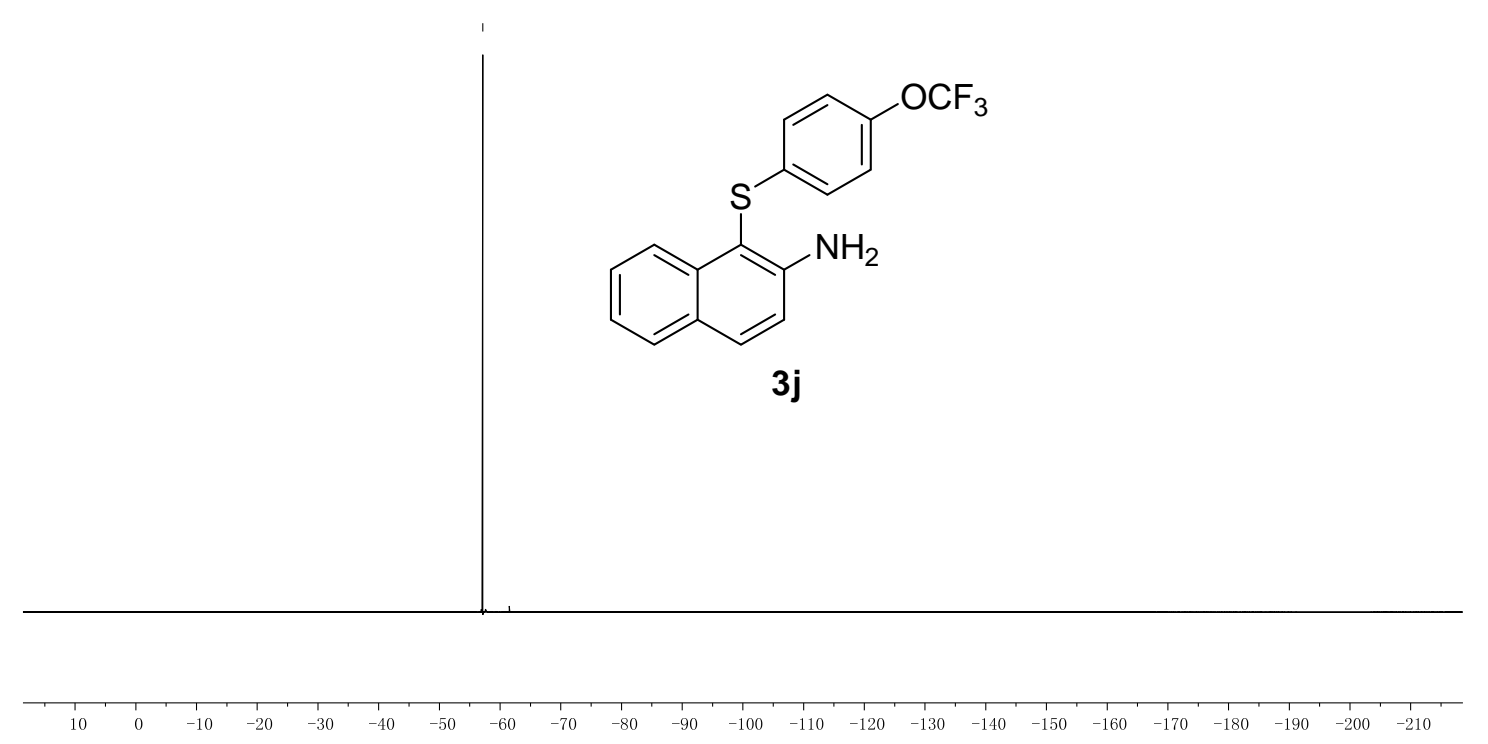

Figure S21

${ }^{1} \mathrm{H}$ and ${ }^{13} \mathrm{C}$ NMR Spectrum of Compound 3k
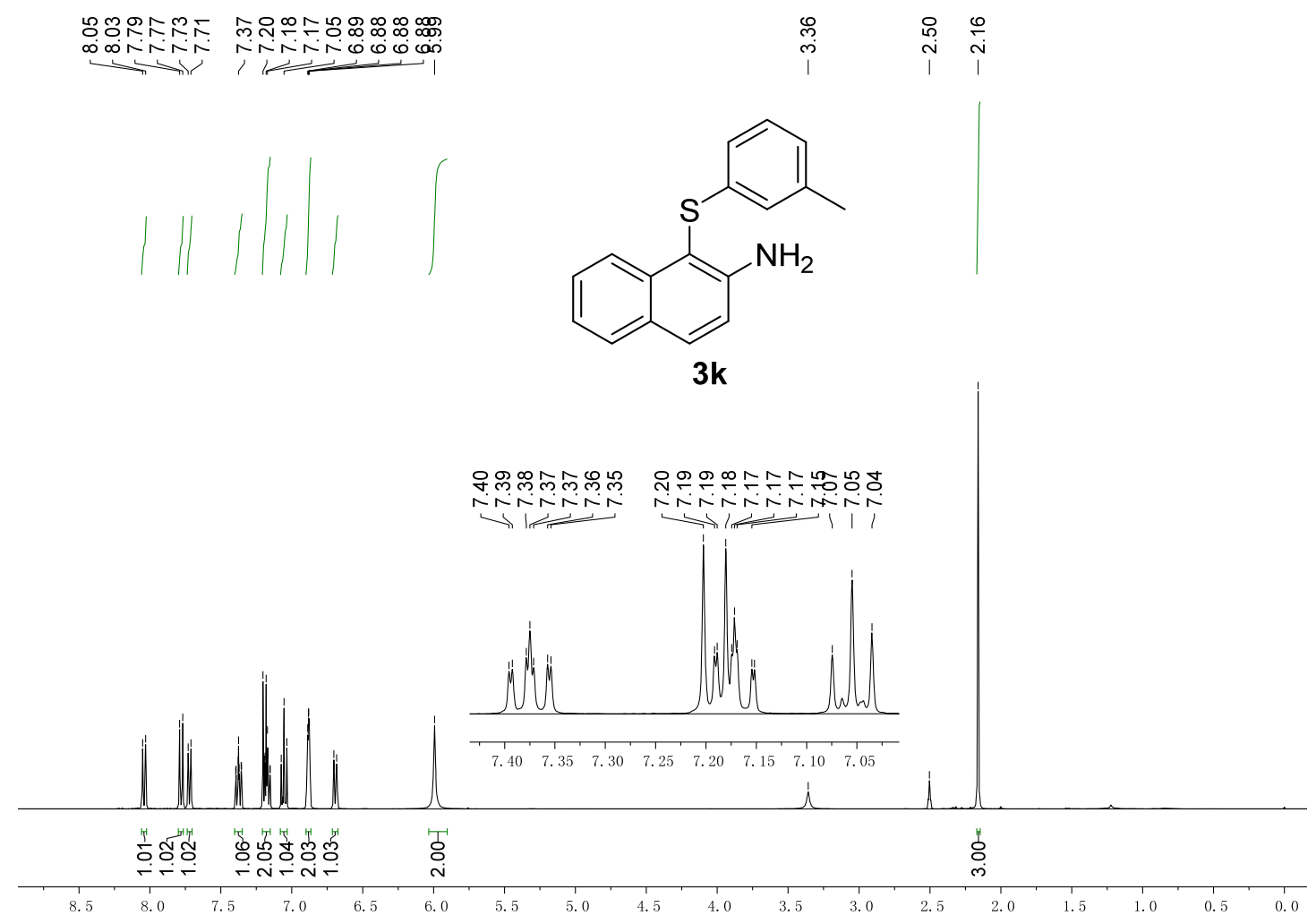

Figure S22 


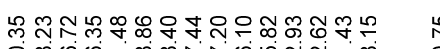

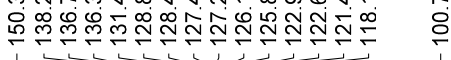
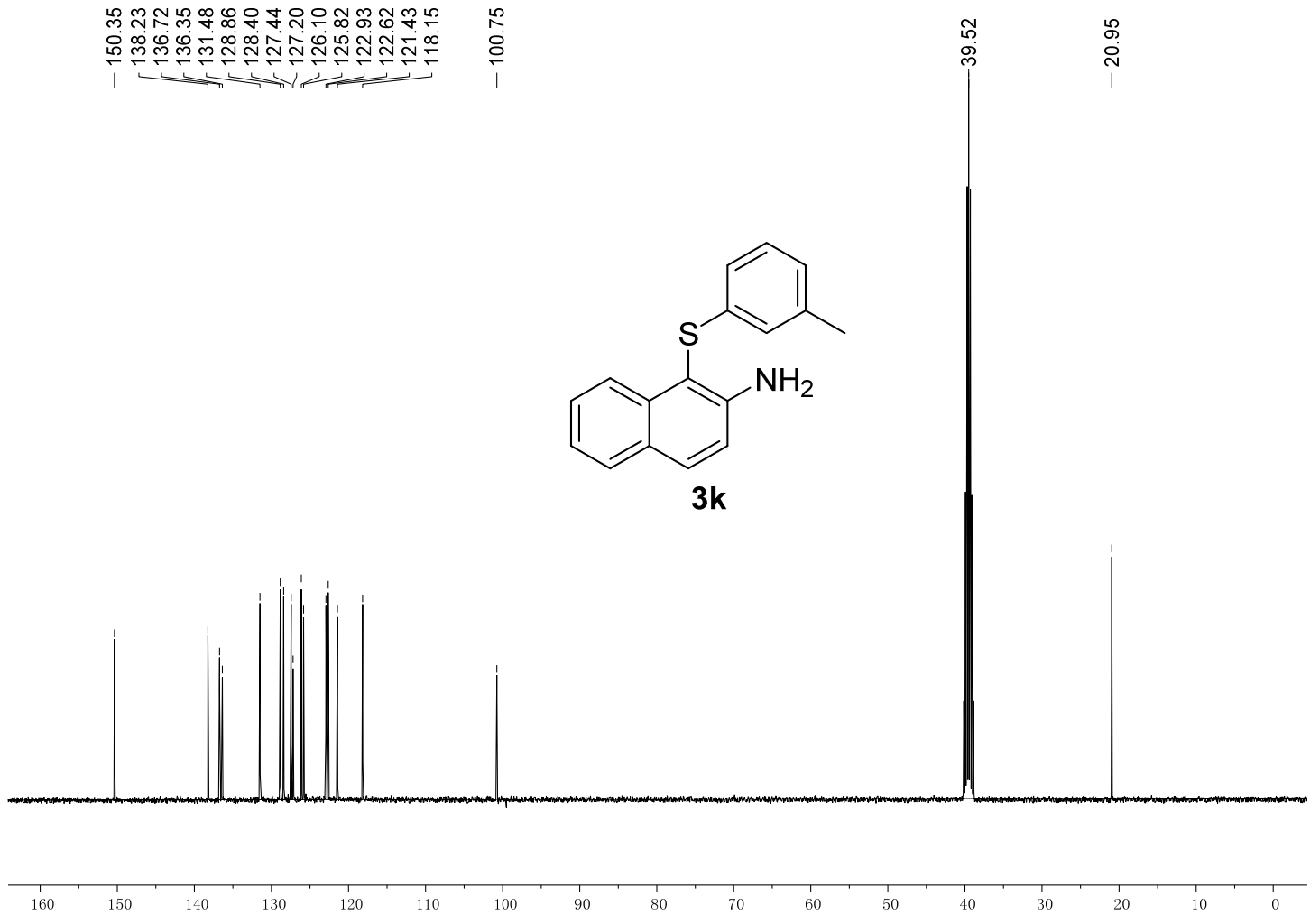

\section{Figure S23}

${ }^{1} \mathrm{H}$ and ${ }^{13} \mathrm{C}$ NMR Spectrum of Compound $3 \mathbf{I}$

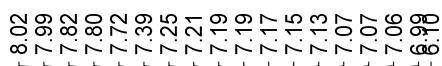

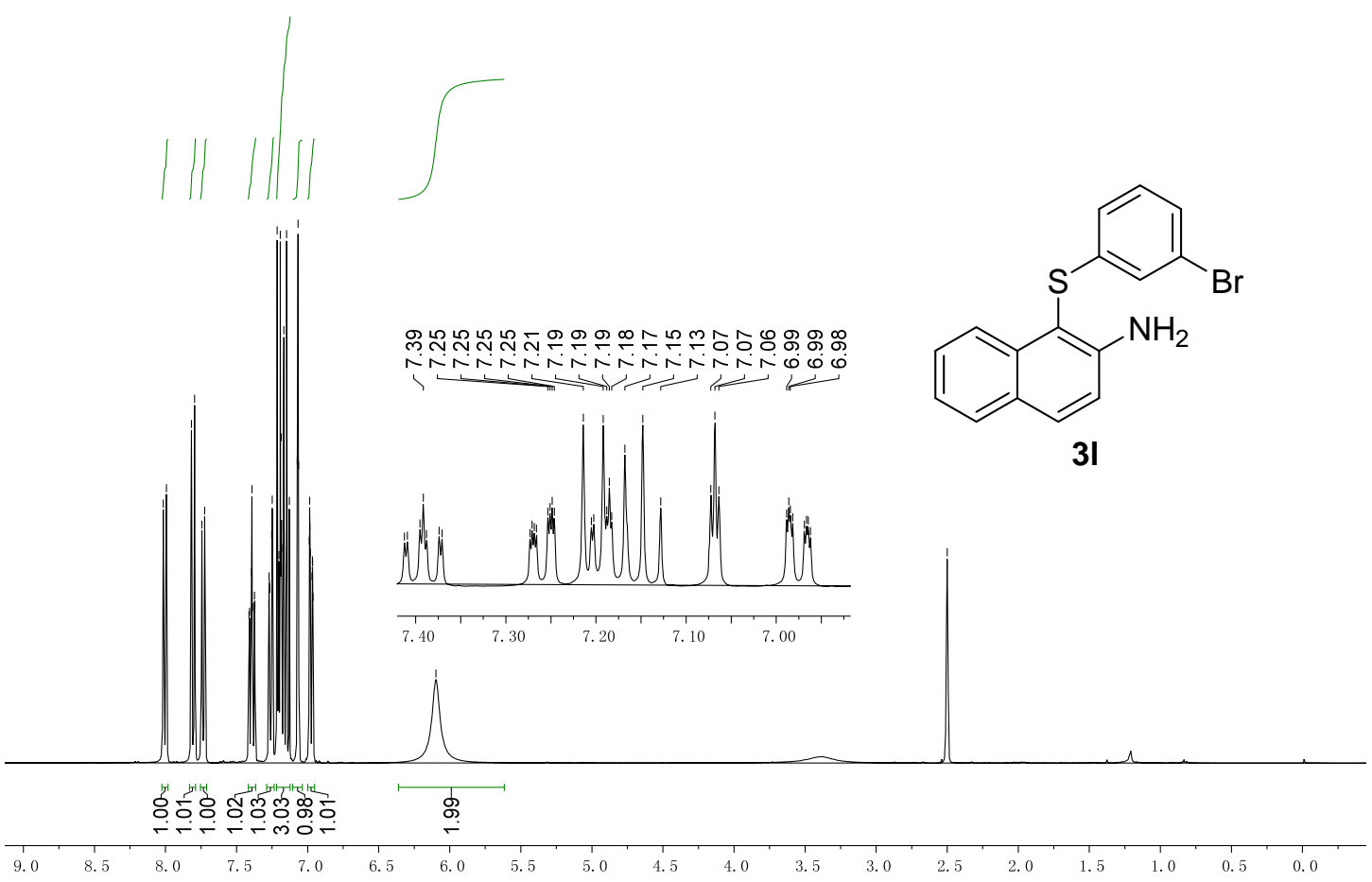

Figure S24 
<smiles>Nc1ccc2ccccc2c1Sc1cccc(Br)c1</smiles>

3I
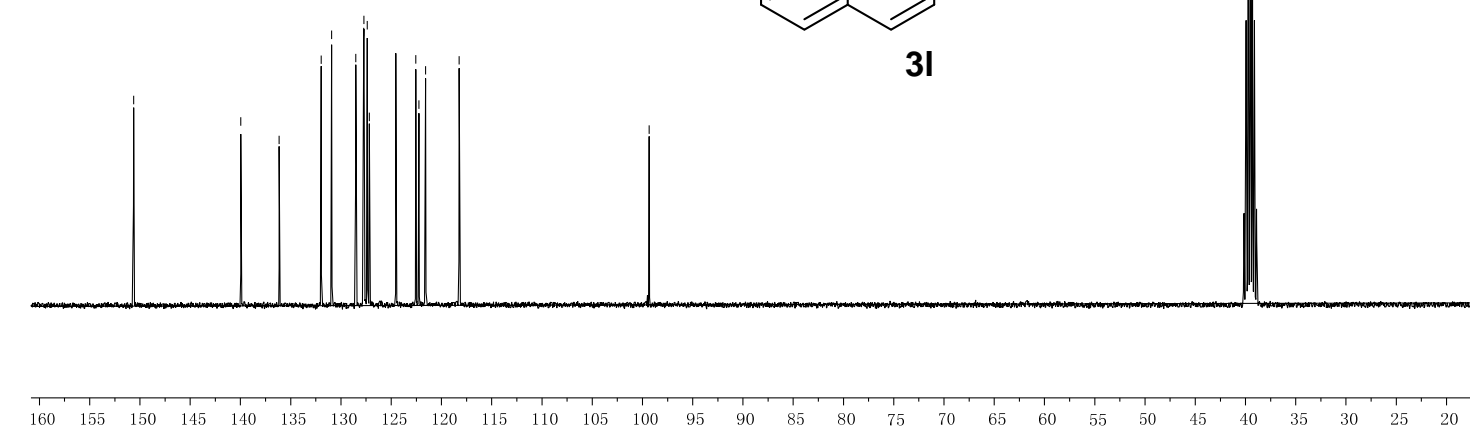

Figure S25

${ }^{1} \mathrm{H}$ and ${ }^{13} \mathrm{C}$ NMR Spectrum of Compound $3 \mathbf{m}$

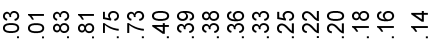

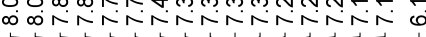

$\stackrel{\circ}{\substack{n \\ i}}$

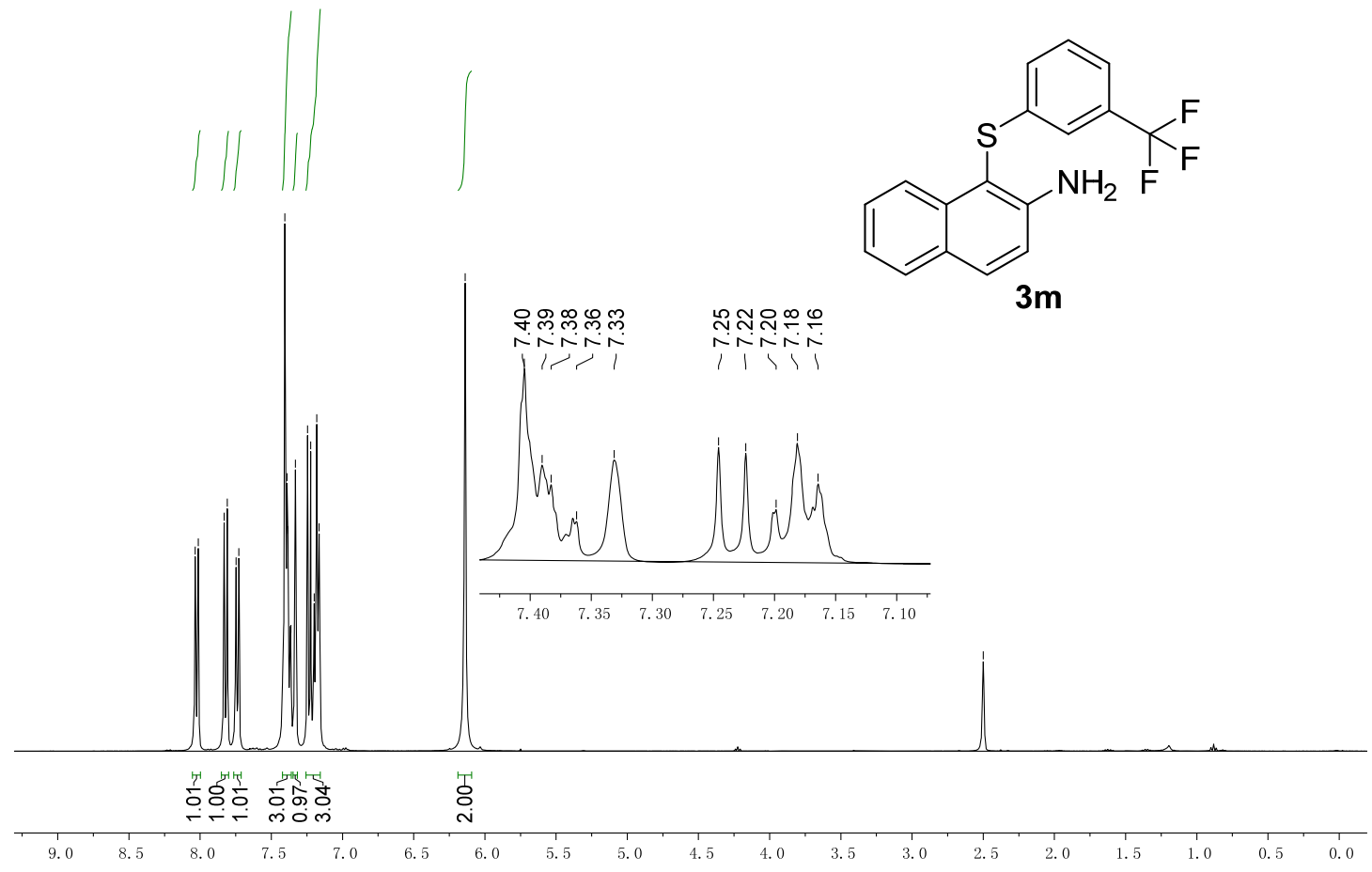

Figure S26 
<smiles>Nc1ccc2ccccc2c1Sc1cccc(C(F)(F)F)c1</smiles>

$3 m$

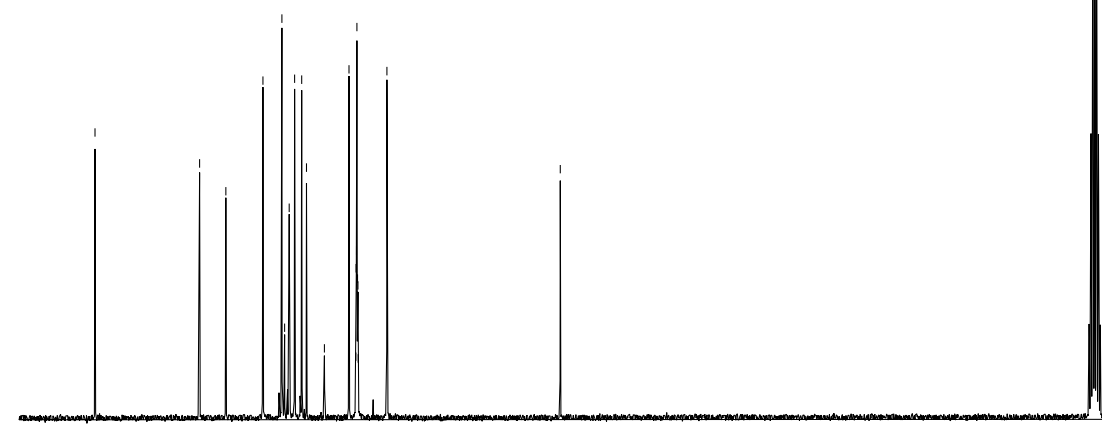

Figure S27

${ }^{1} \mathrm{H}$ and ${ }^{13} \mathrm{C}$ NMR Spectrum of Compound 3n

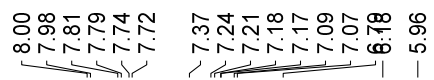<smiles>Cc1ccc(C)c(Sc2c(N)ccc3ccccc23)c1</smiles>

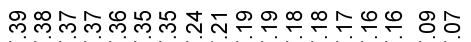

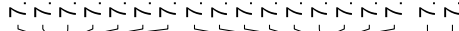
乎

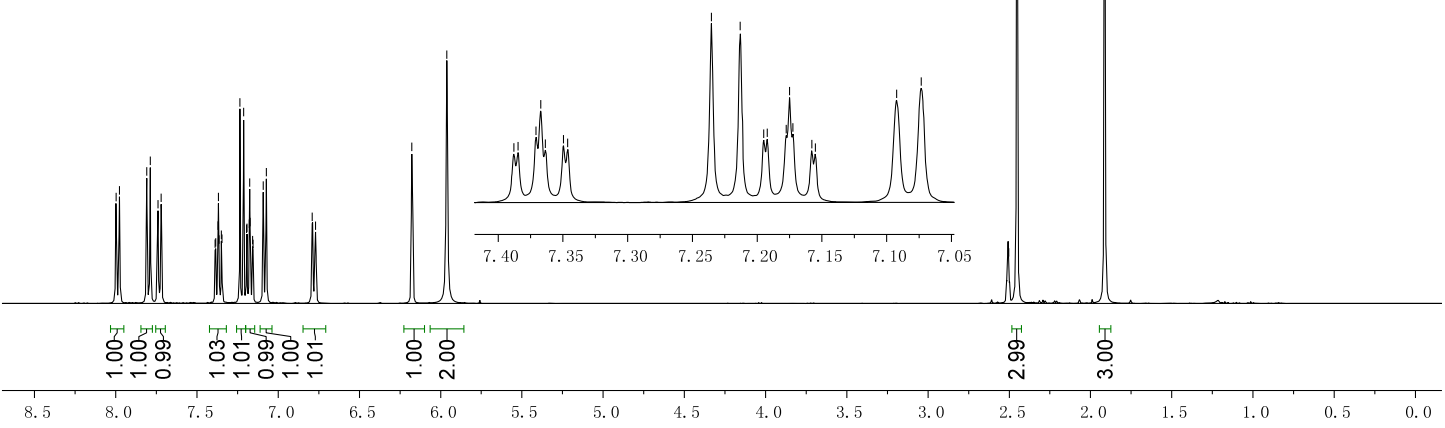

Figure S28 

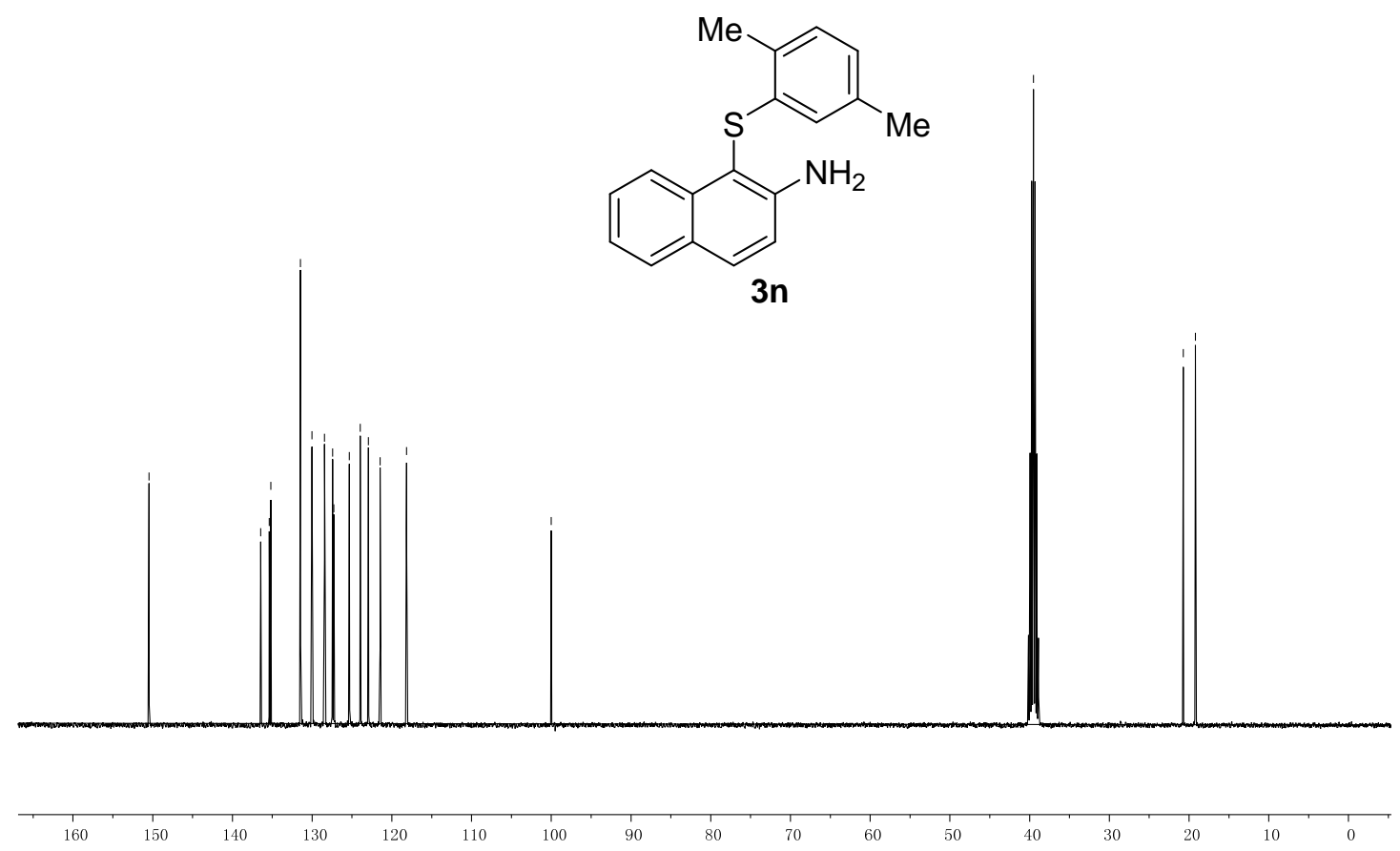

Figure S29

${ }^{1} \mathrm{H}$ and ${ }^{13} \mathrm{C}$ NMR Spectrum of Compound 3 o

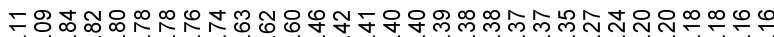

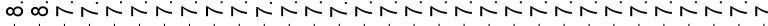

$\stackrel{\text { m }}{\stackrel{5}{i \infty}}$
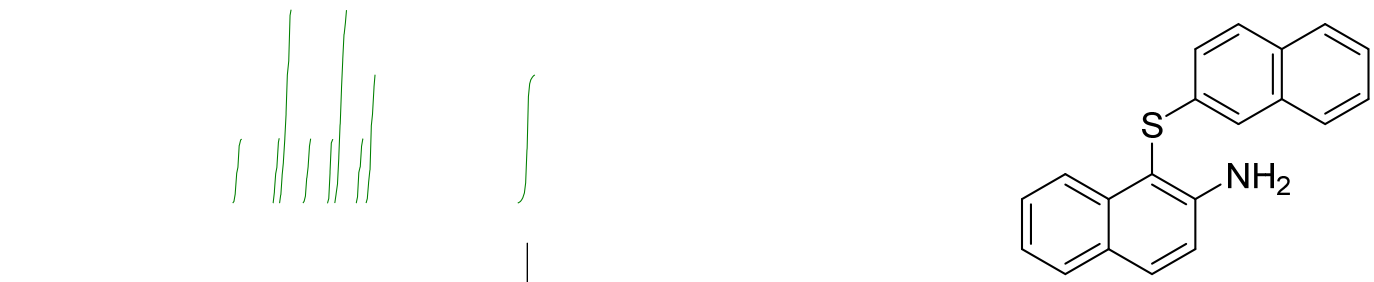

30

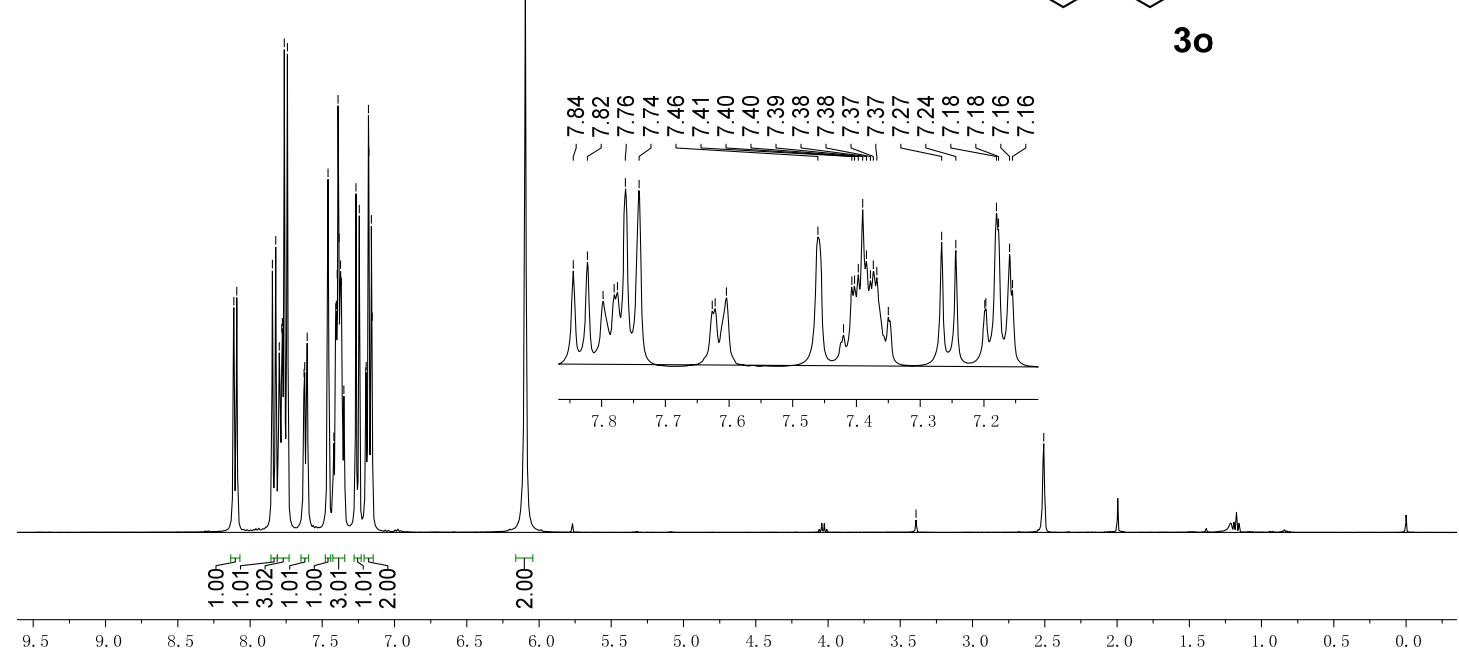

Figure S30 


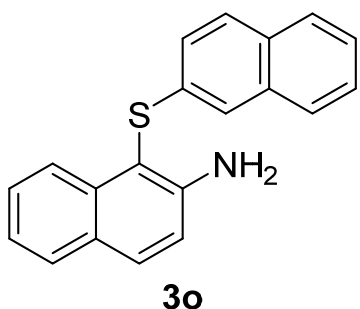

$\begin{array}{lllllllllllllllllllllllllllllll}165 & 160 & 155 & 150 & 145 & 140 & 135 & 130 & 125 & 120 & 115 & 110 & 105 & 100 & 95 & 90 & 85 & 80 & 75 & 70 & 65 & 60 & 55 & 50 & 45 & 40 & 35 & 3(\end{array}$

Figure S31

${ }^{1} \mathrm{H}$ and ${ }^{13} \mathrm{C}$ NMR Spectrum of Compound 3p

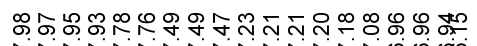
NNNNNNNNNNNNGG60

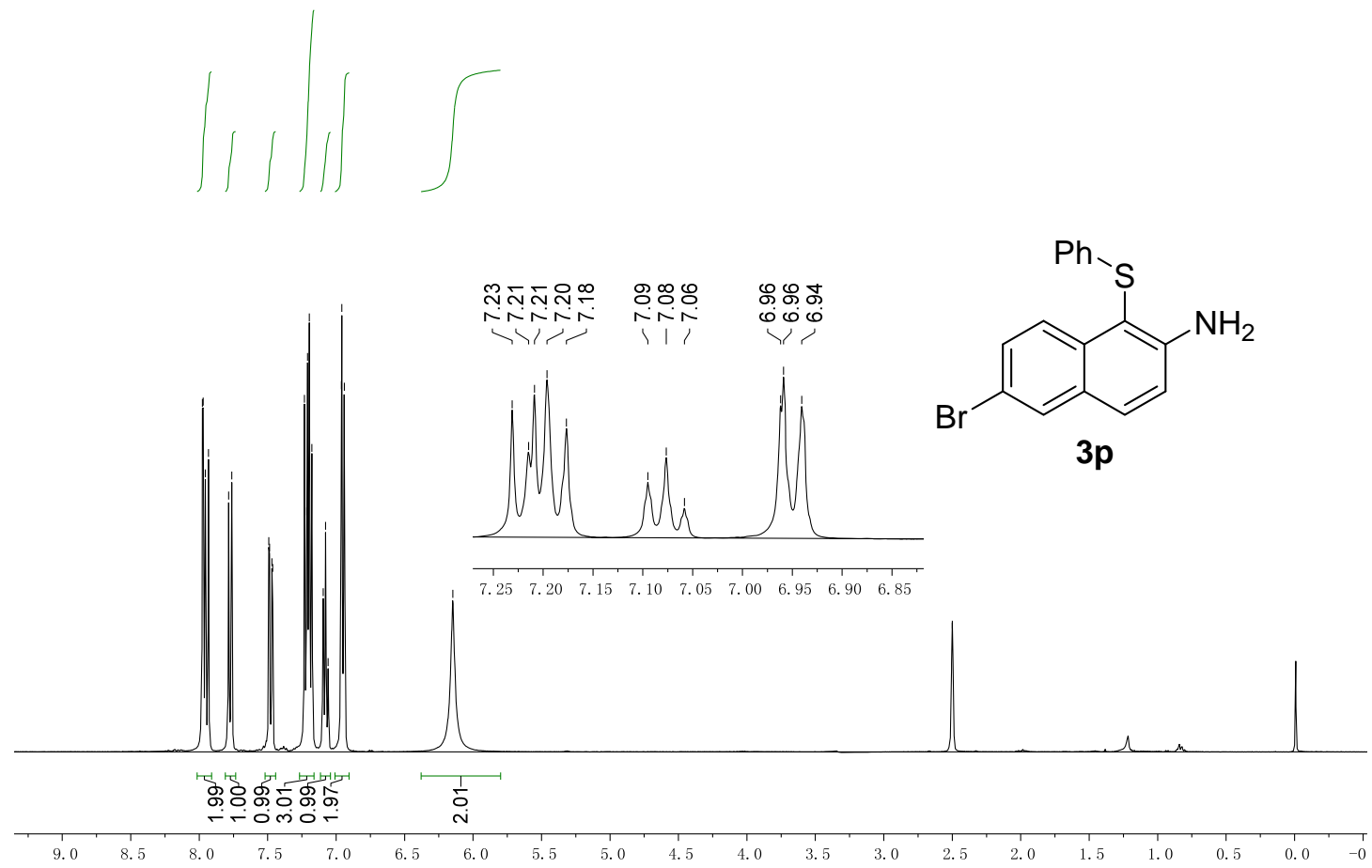

Figure S32 


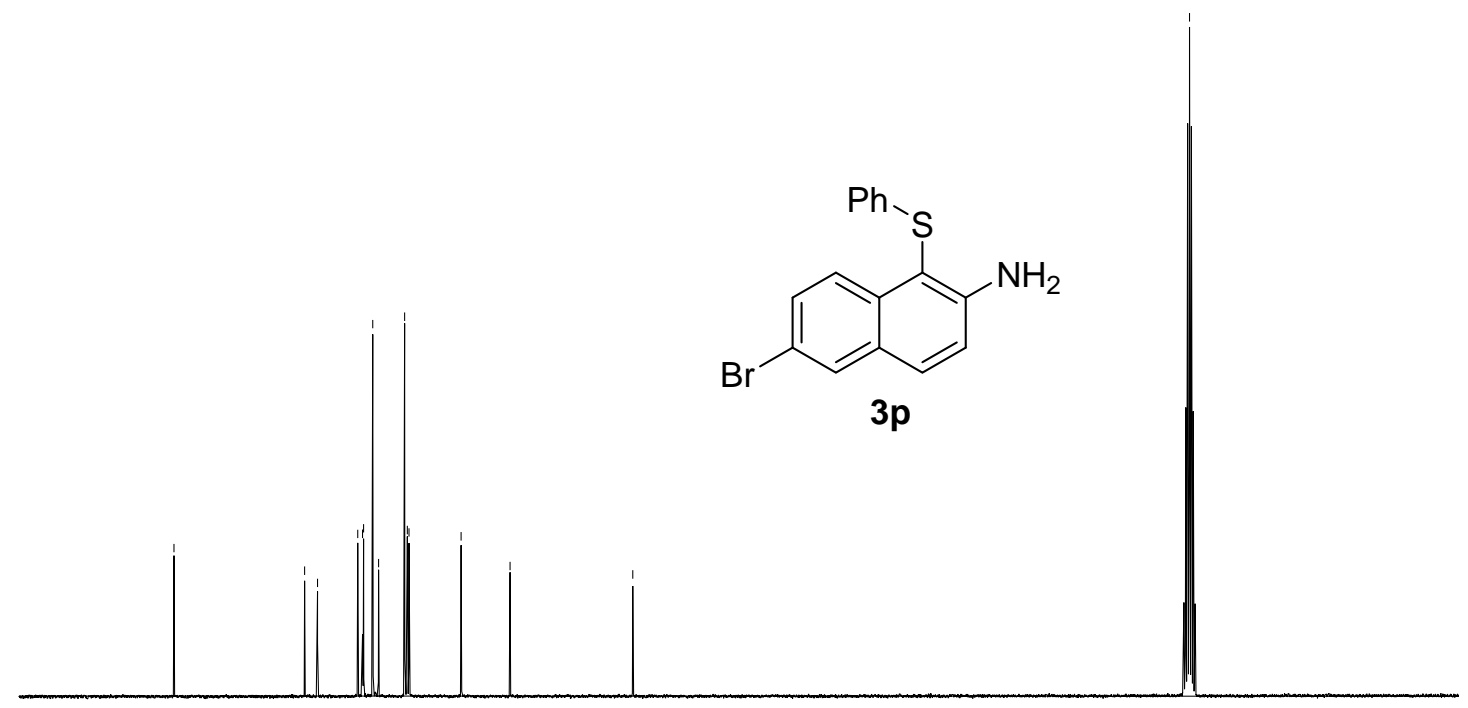

Figure S33

${ }^{1} \mathrm{H}$ and ${ }^{13} \mathrm{C}$ NMR Spectrum of Compound $\mathbf{3 q}$
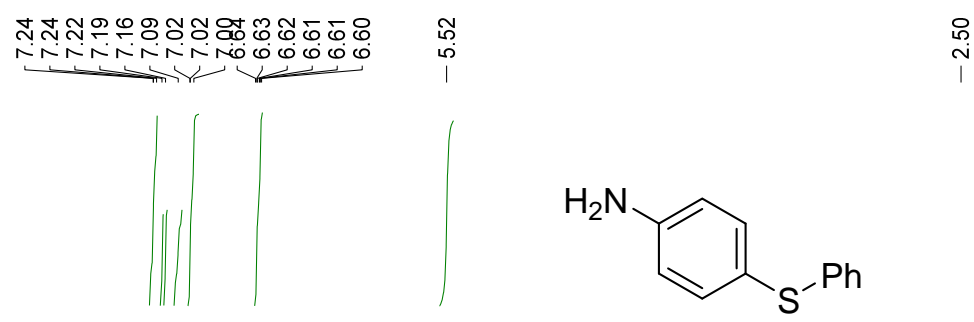

$3 q$

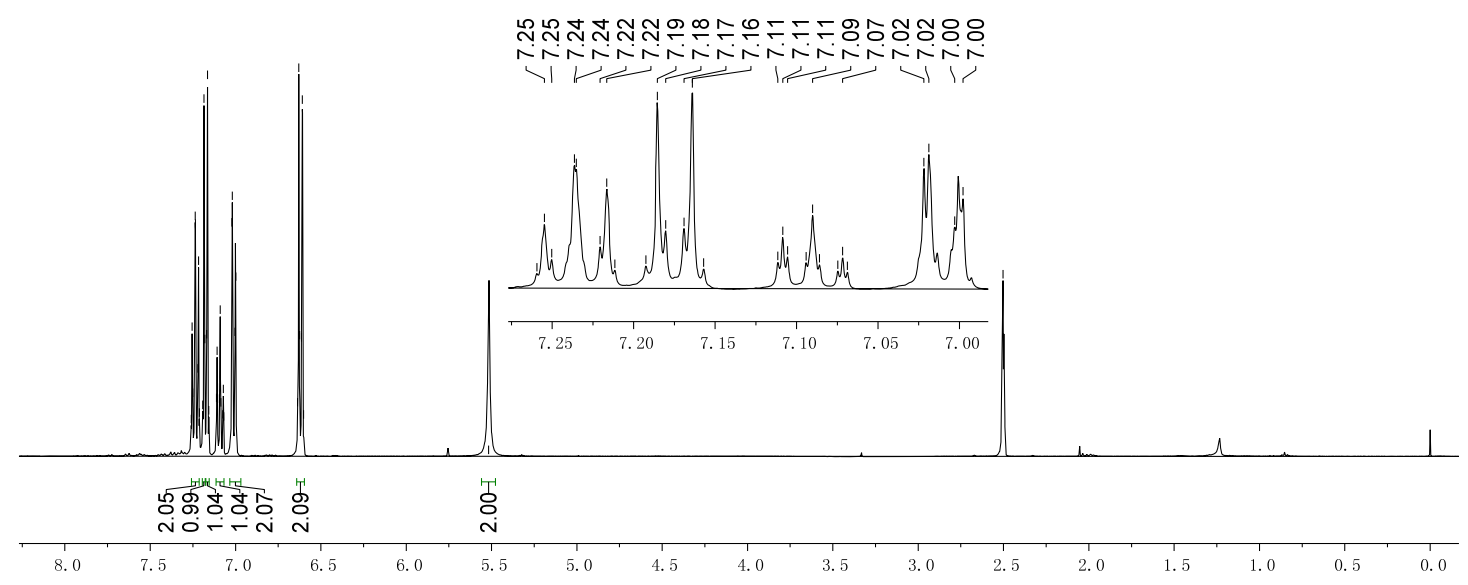

Figure S34 


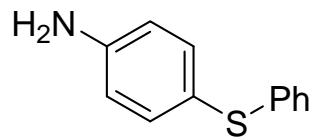

$3 q$

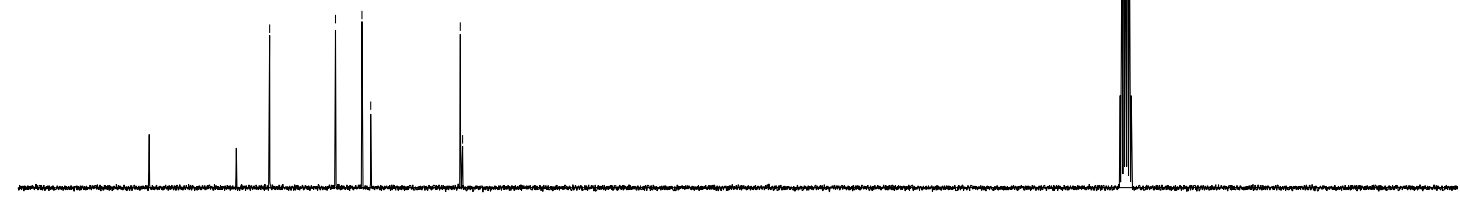

Figure S35

${ }^{1} \mathrm{H}$ and ${ }^{13} \mathrm{C}$ NMR Spectrum of Compound 3r

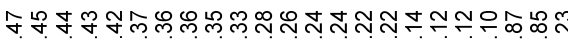

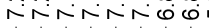

$\stackrel{\leftrightarrow}{\text { i }}$

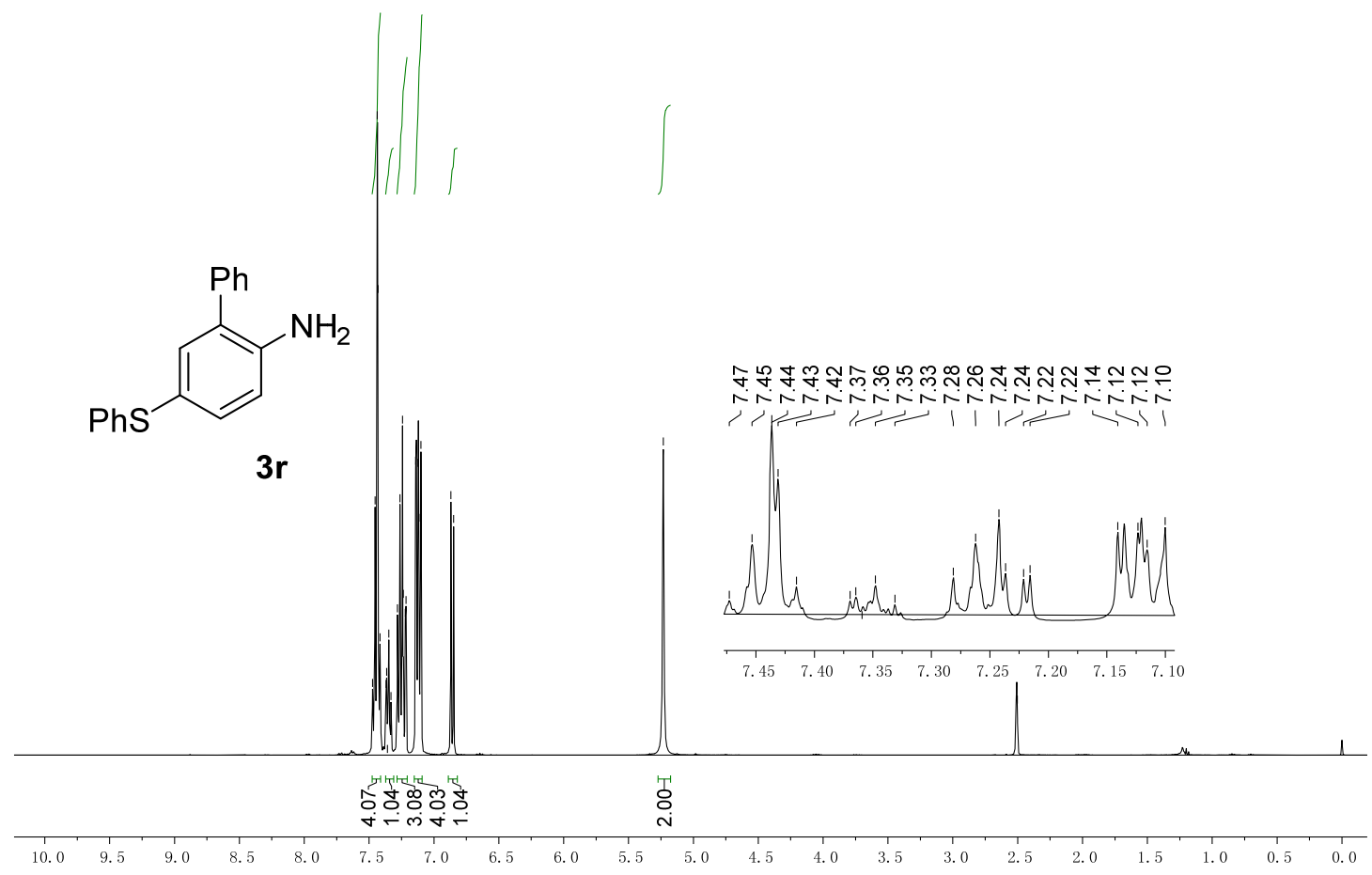

Figure S36 


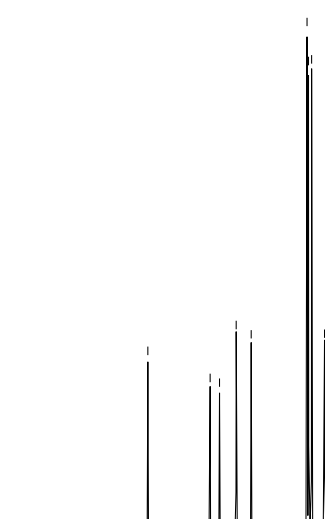<smiles>Nc1ccc(S)cc1-c1ccccc1</smiles>

$3 r$

Figure S37

${ }^{1} \mathrm{H}$ and ${ }^{13} \mathrm{C}$ NMR Spectrum of Compound 4a

$$
\text { m }
$$

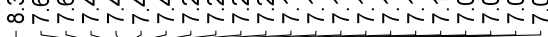

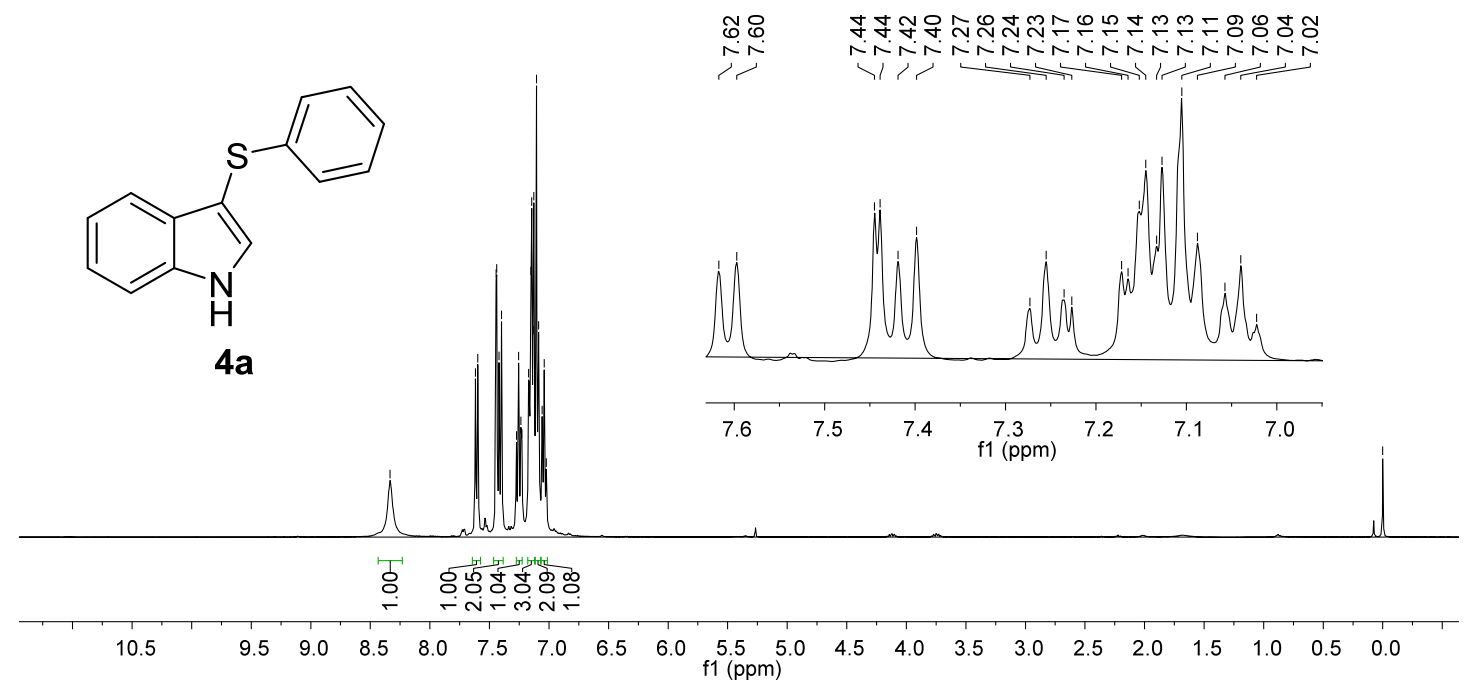

Figure S38 

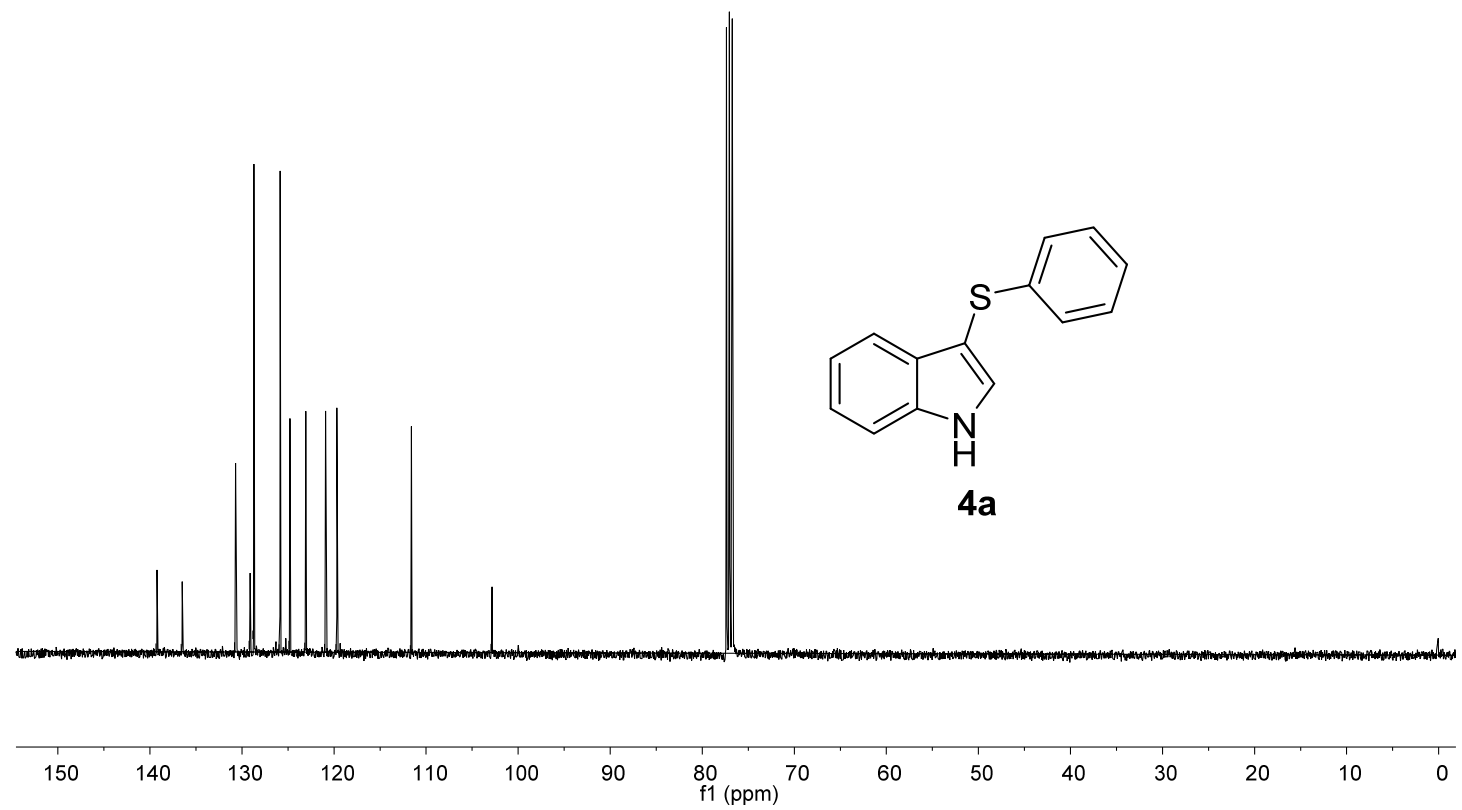

Figure S39

${ }^{1} \mathrm{H},{ }^{13} \mathrm{C}$ NMR and ${ }^{19} \mathrm{~F}$ NMR Spectrum of Compound $\mathbf{4 b}$

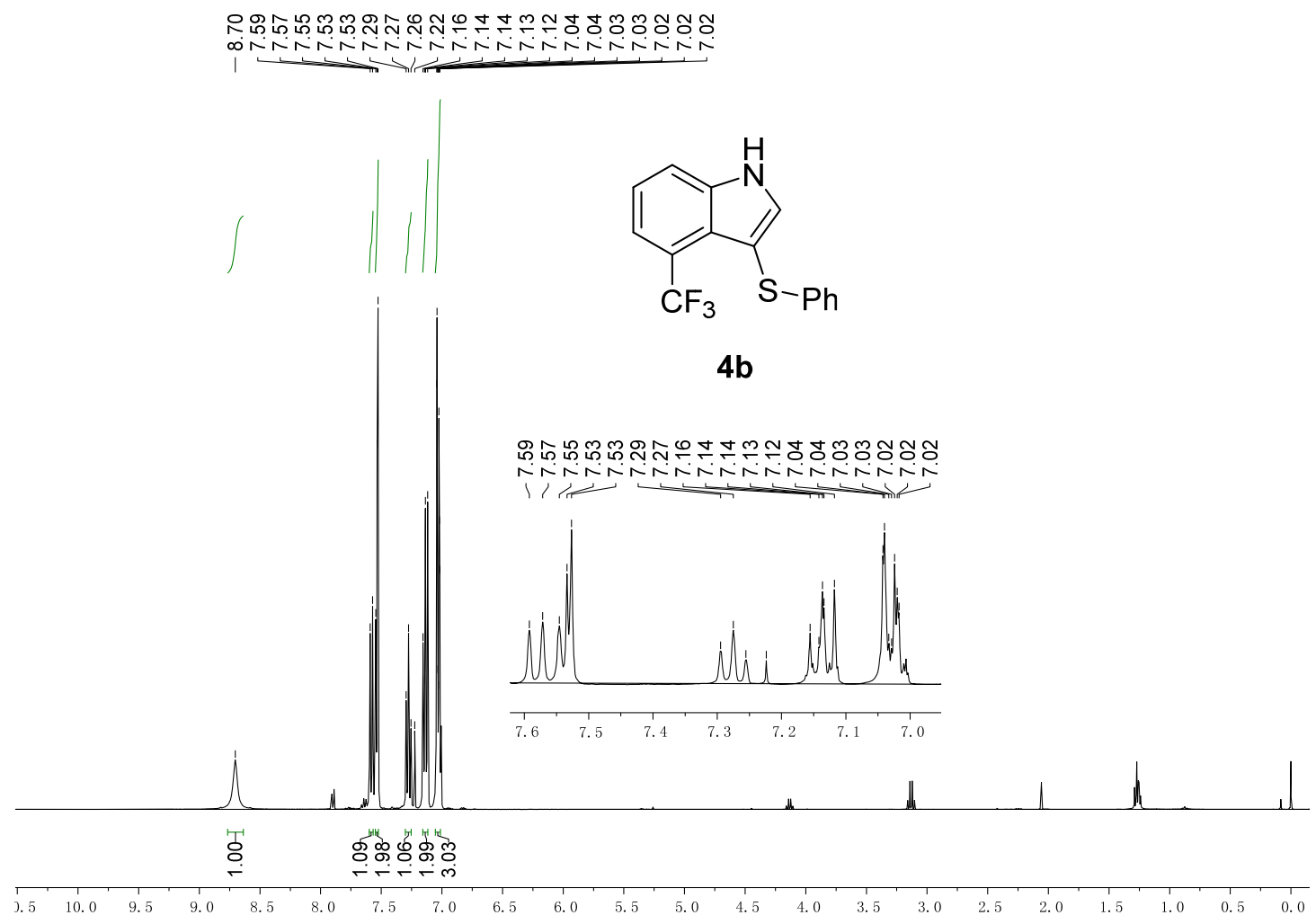

Figure S40 


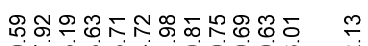

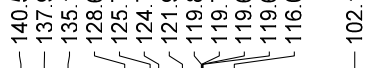

$\frac{0}{i}$<smiles>FC(F)(F)c1cccc2[nH]cc(Sc3ccccc3)c12</smiles>

4b
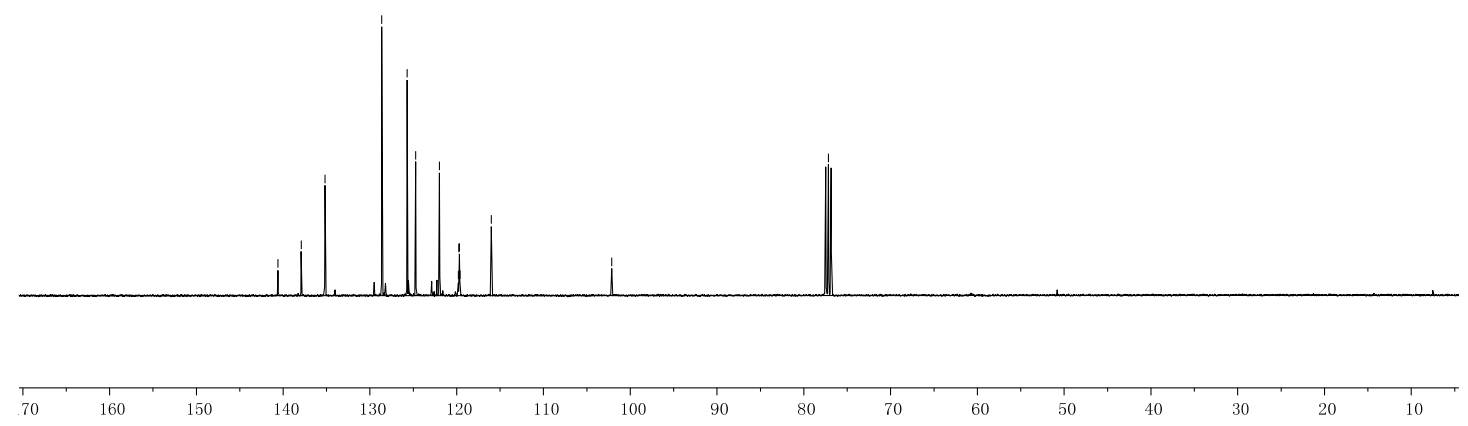

Figure S41

in

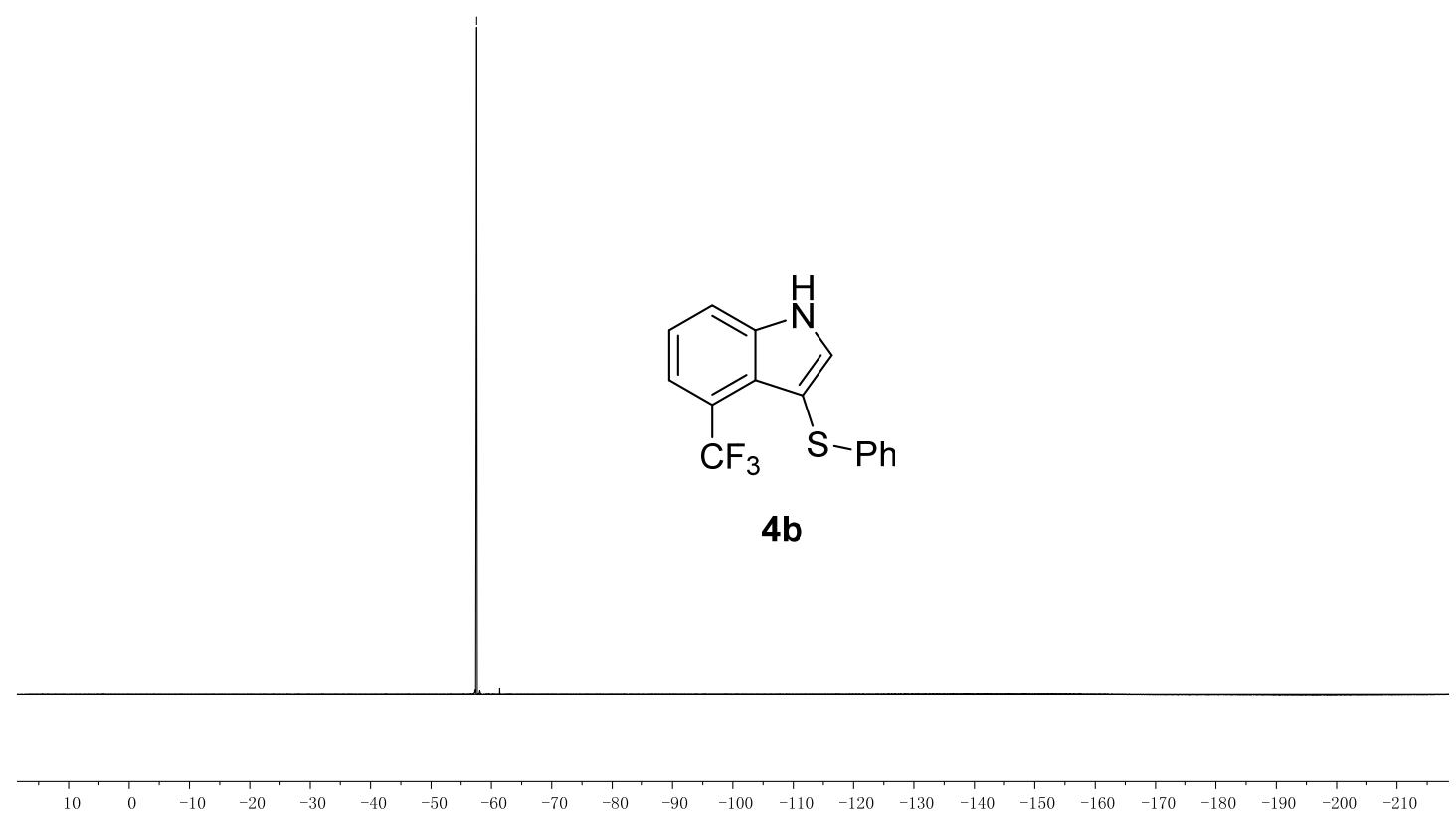

Figure S42 
${ }^{1} \mathrm{H}$ and ${ }^{13} \mathrm{C}$ NMR Spectrum of Compound $4 \mathrm{c}$

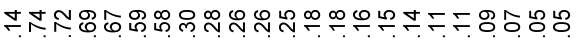

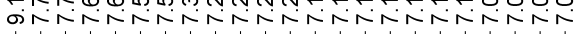

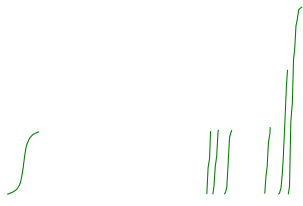<smiles>CC(C)(C)c1c[nH]c(Sc2ccccc2)c1[N+](=O)[O-]</smiles>

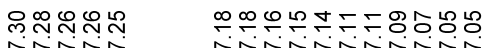

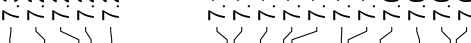

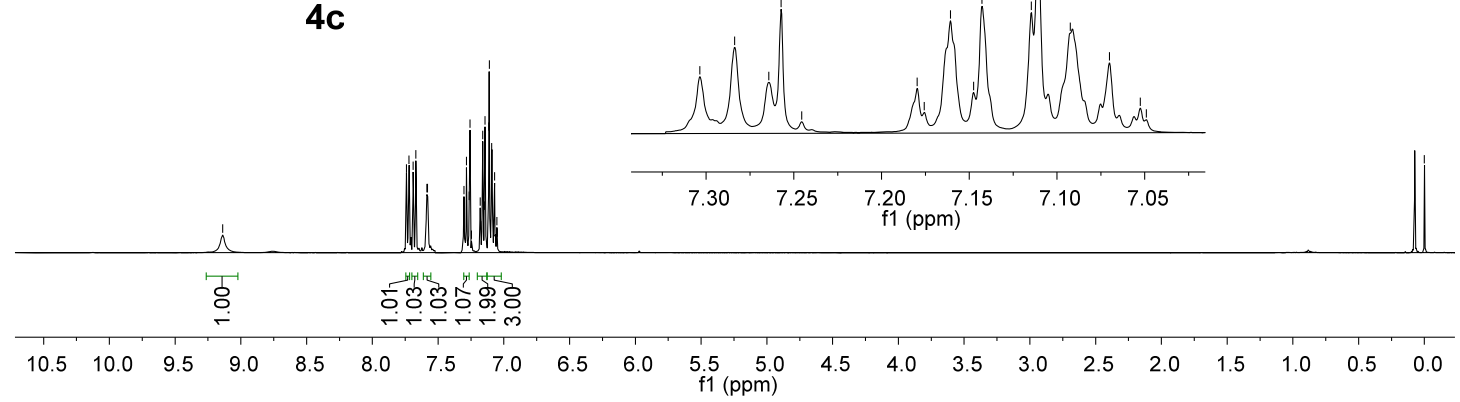

Figure S43

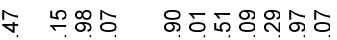

焉

ํㅠㅁ

$\underset{\substack{\infty \\ i}}{\stackrel{0}{1}}$<smiles>O=[N+]([O-])c1cccc2[nH]cc(Sc3ccccc3)c12</smiles>

4c

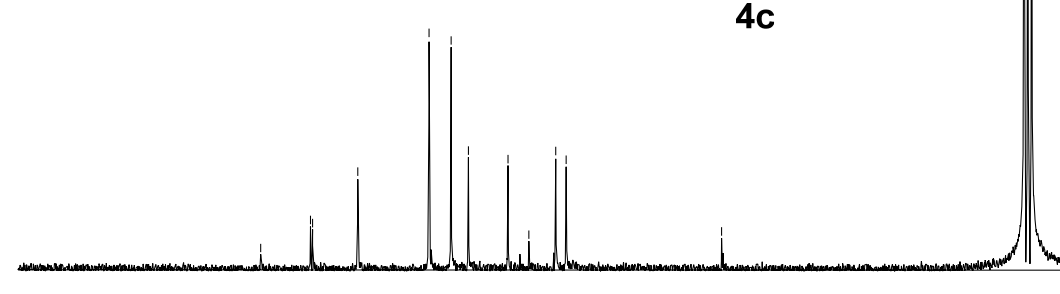

155

$145 \quad 135$

125

$\begin{array}{lllllllllllllll}115 & 110 & 105 & 95 & 90 & 85 & 80 & 75 & 70 & 65 & 60 & 55 & 50 & 45 & 4\end{array}$

Figure S44 
${ }^{1} \mathrm{H}$ and ${ }^{13} \mathrm{C}$ NMR Spectrum of Compound 4d

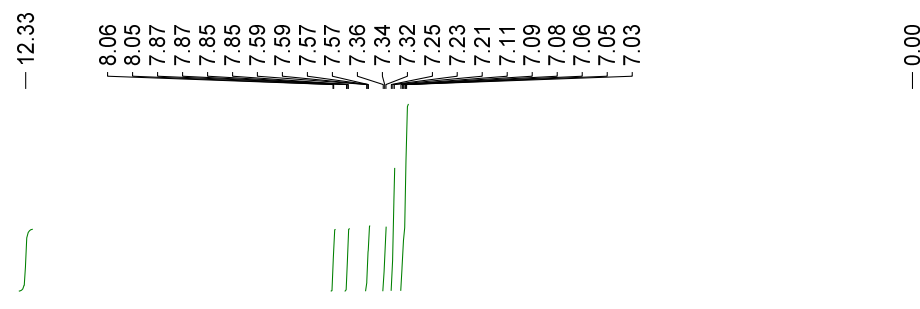

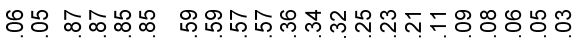

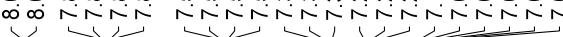

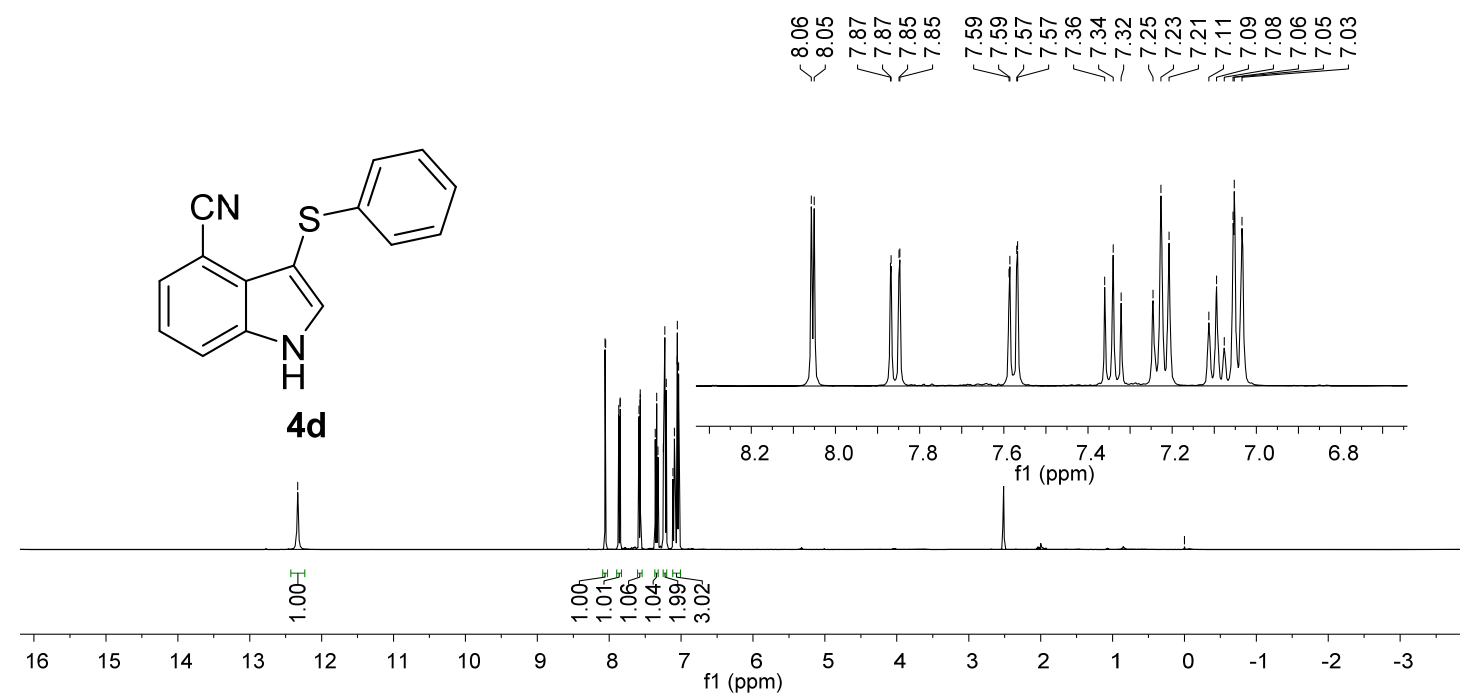

Figure S45

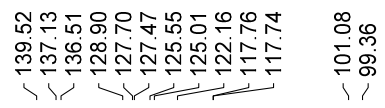

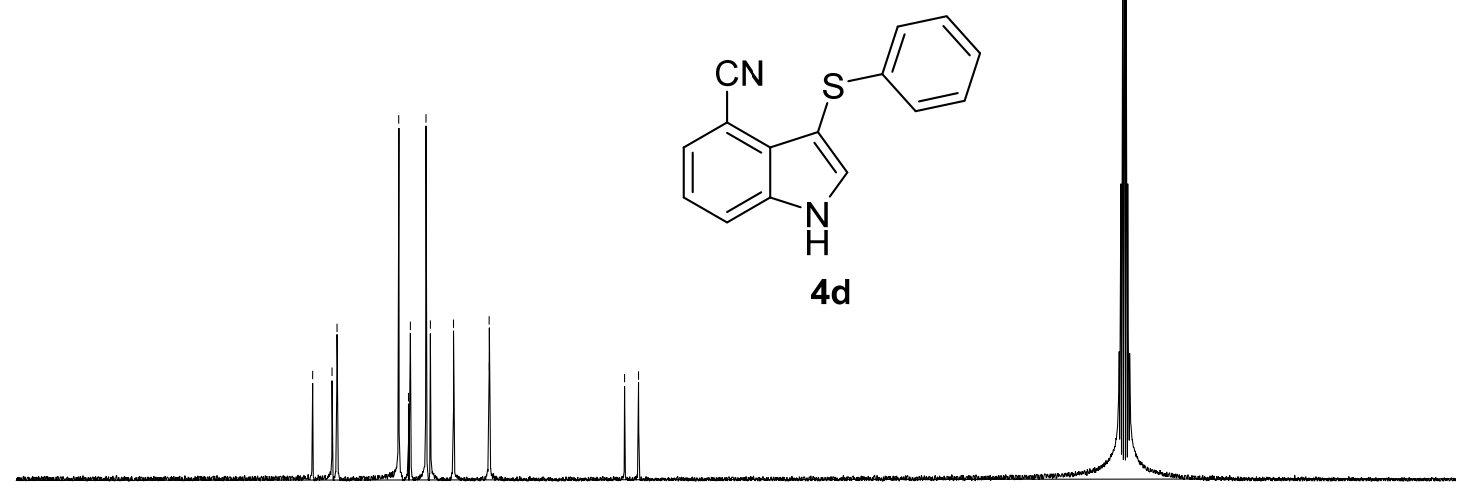

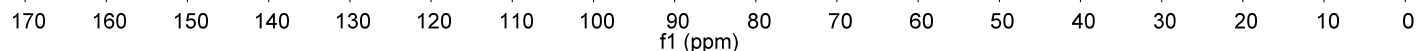

Figure S46 
${ }^{1} \mathrm{H}$ and ${ }^{13} \mathrm{C}$ NMR Spectrum of Compound $4 \mathbf{e}$

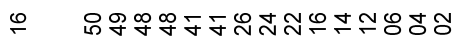

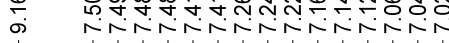

$\stackrel{8}{i}$
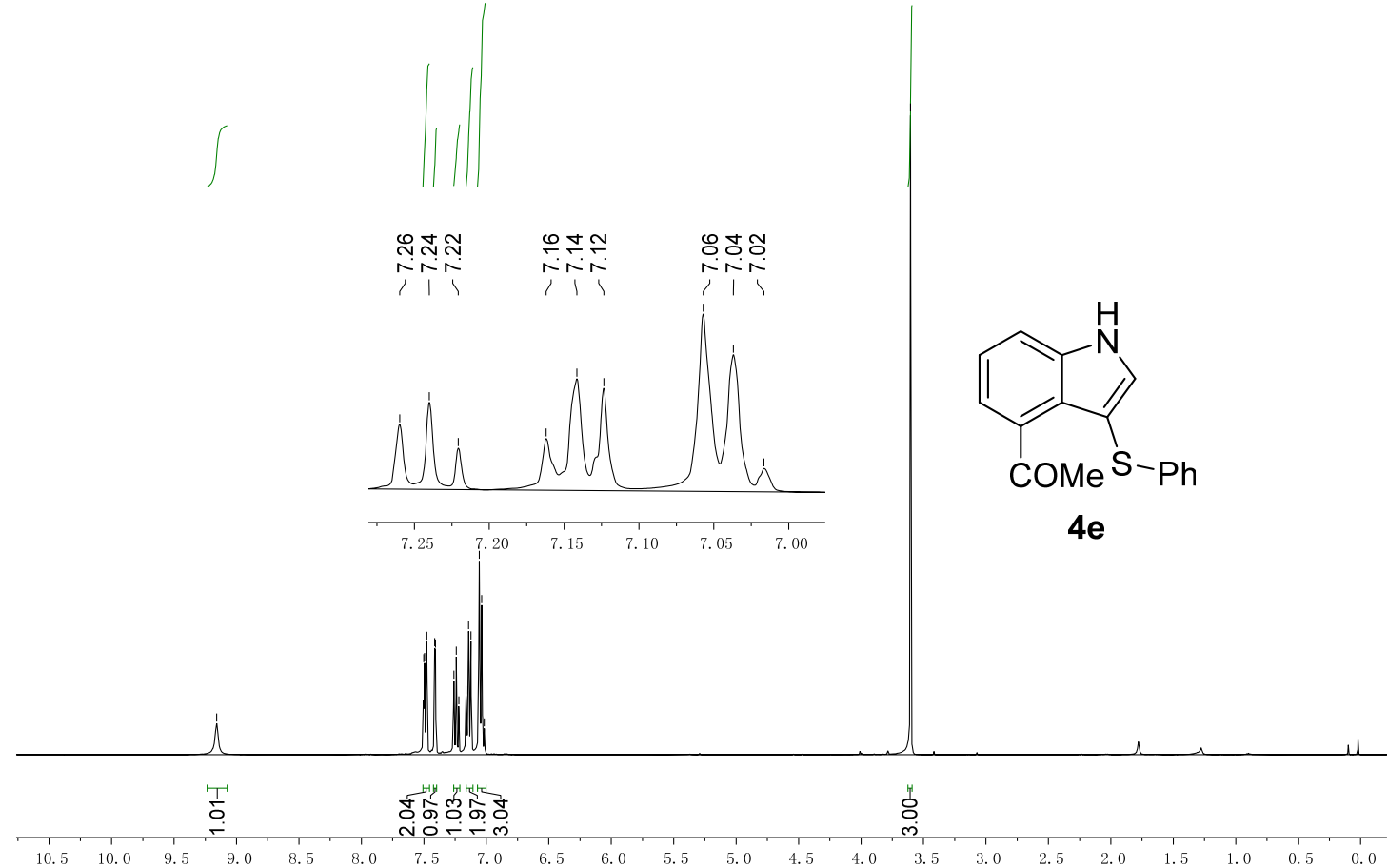

Figure S47

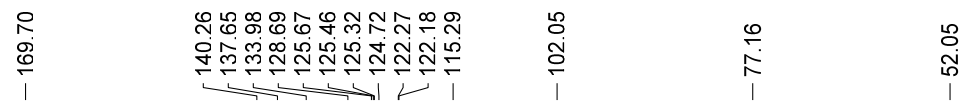
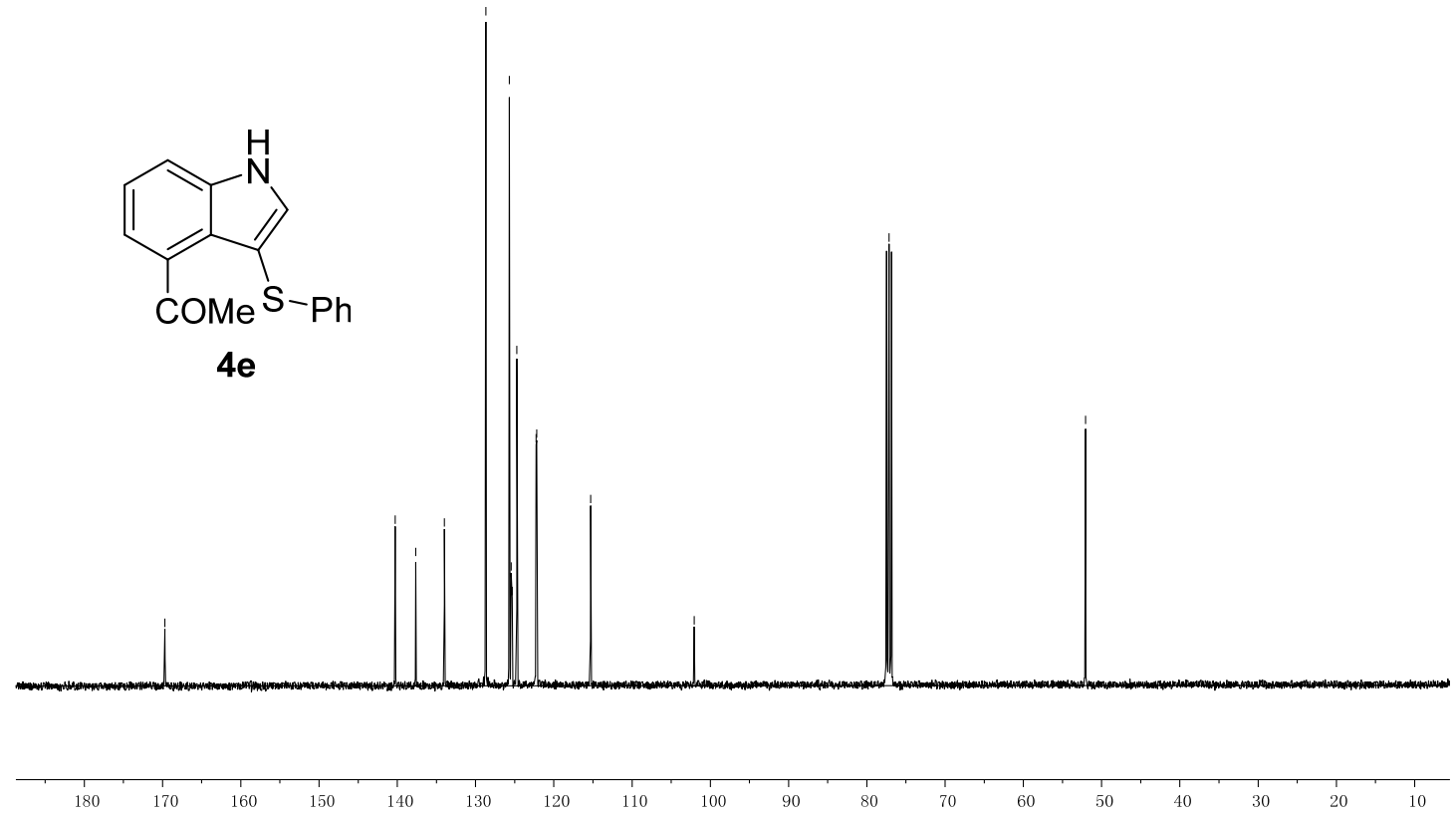

Figure S48 
${ }^{1} \mathrm{H}$ and ${ }^{13} \mathrm{C}$ NMR Spectrum of Compound $\mathbf{4 f}$

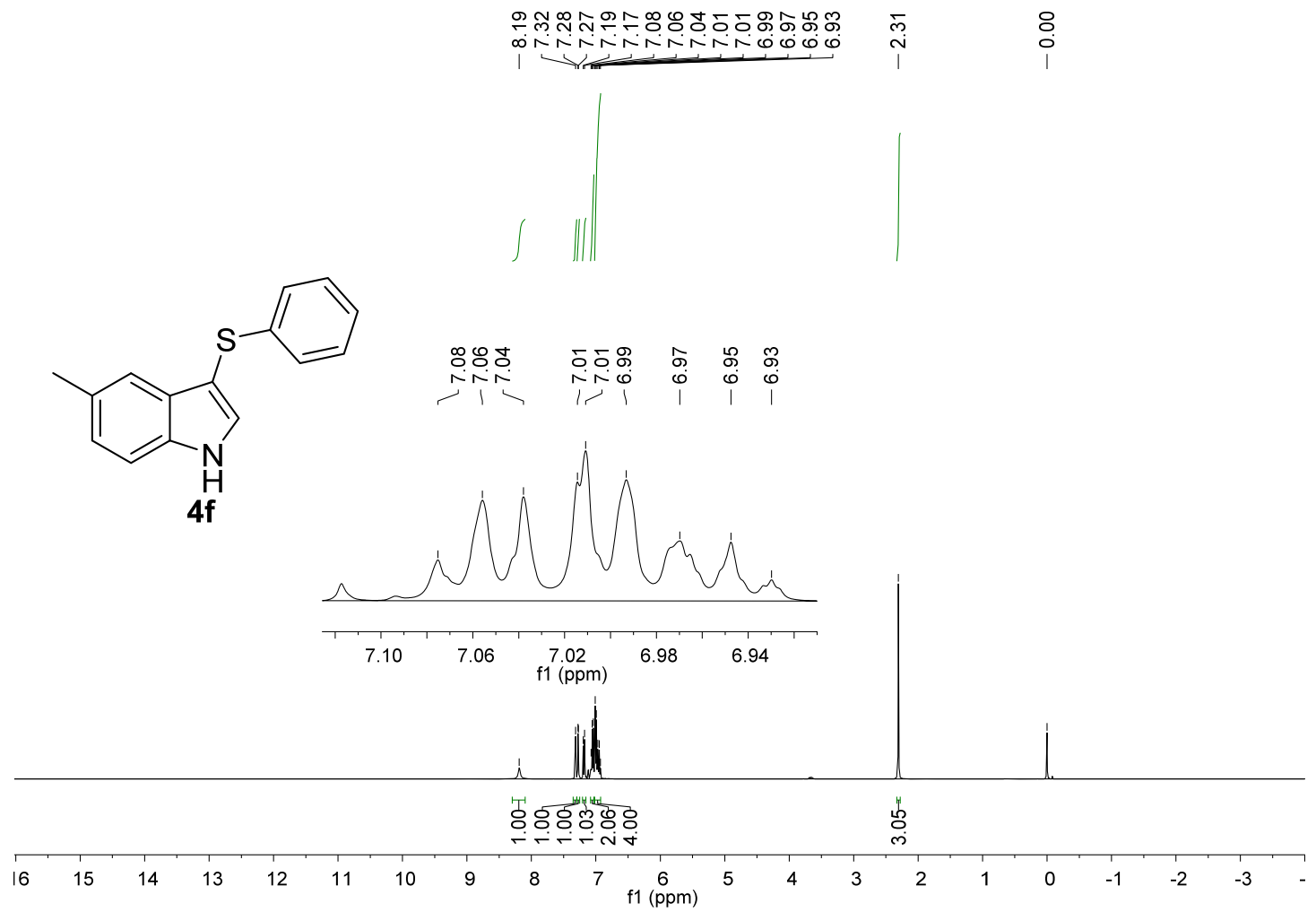

Figure S49

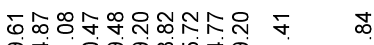

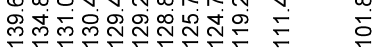

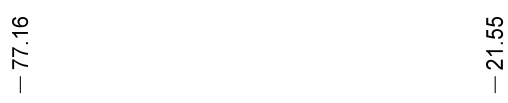

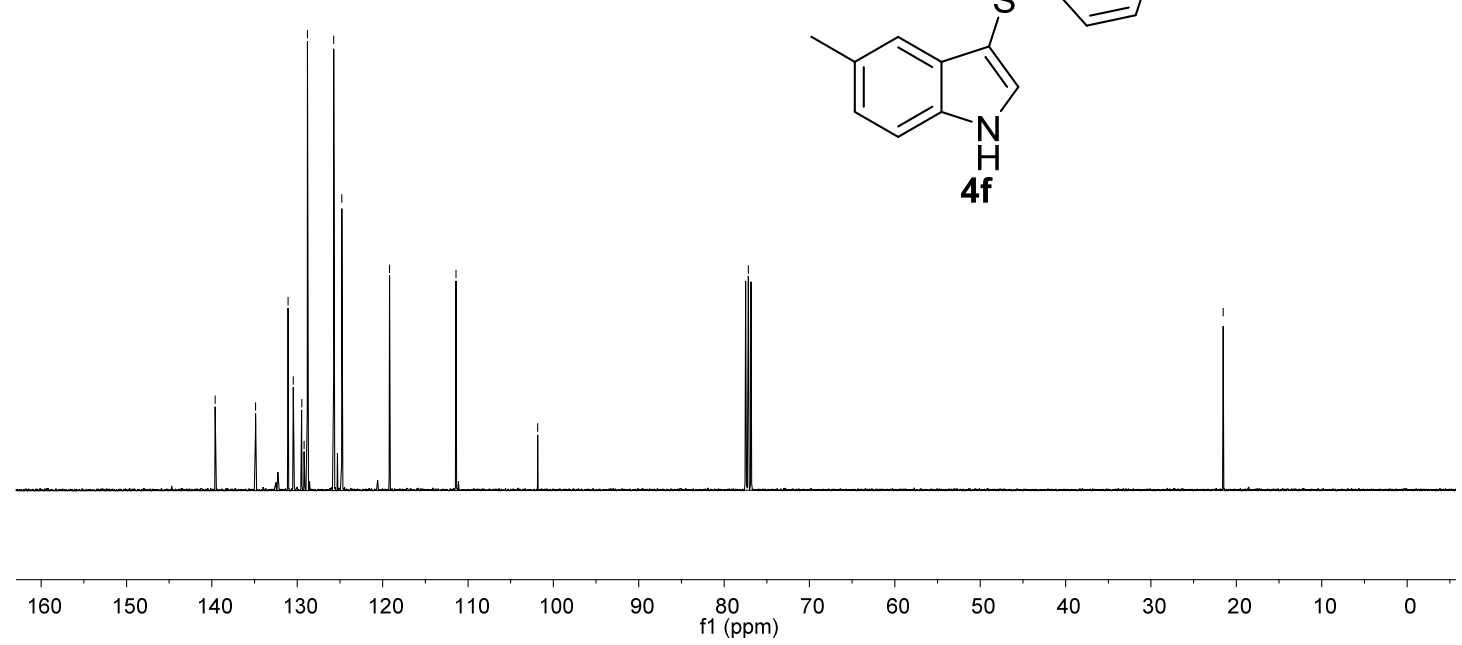

Figure S50 
${ }^{1} \mathrm{H}$ and ${ }^{13} \mathrm{C}$ NMR Spectrum of Compound $\mathbf{4 g}$

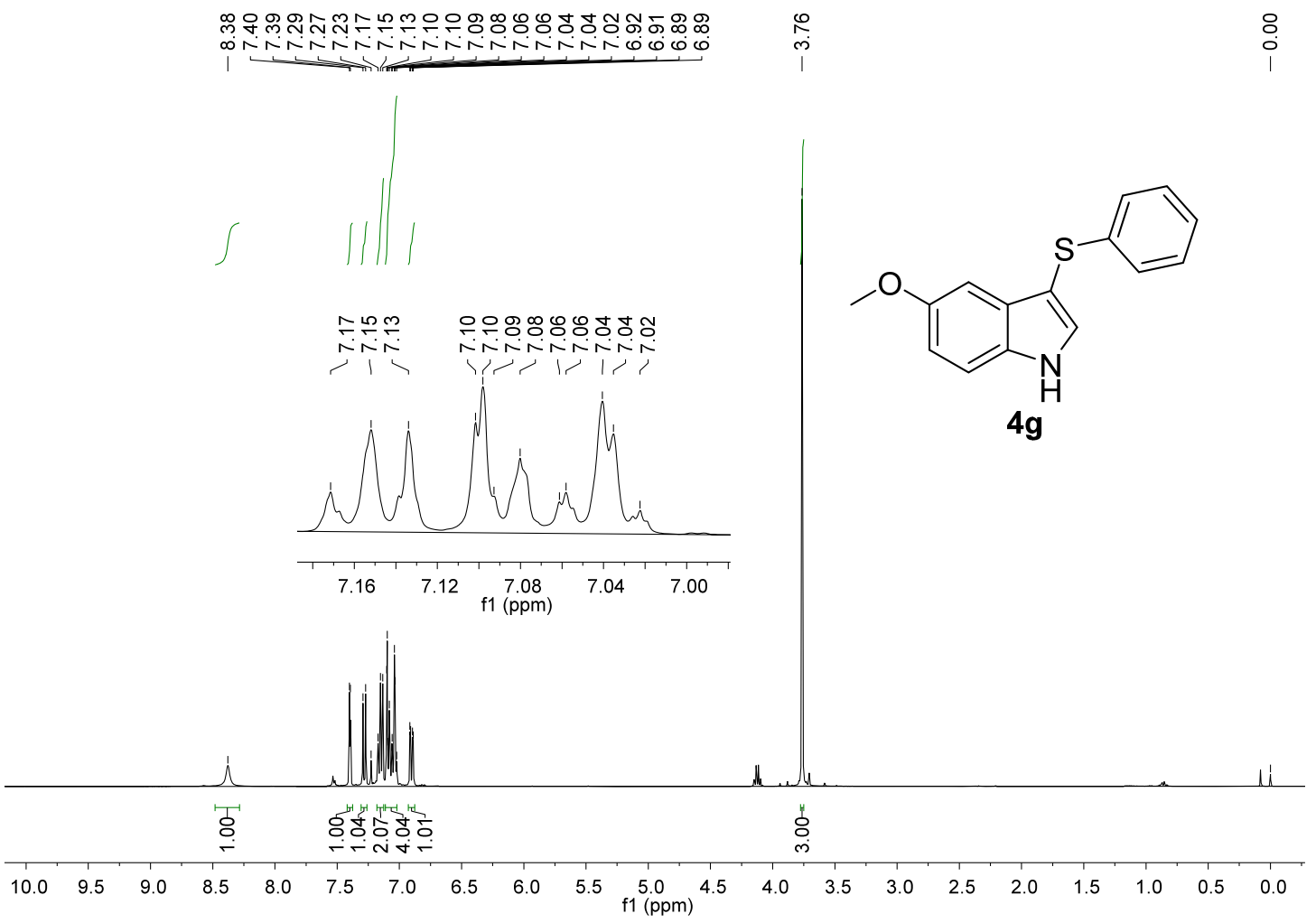

Figure S51

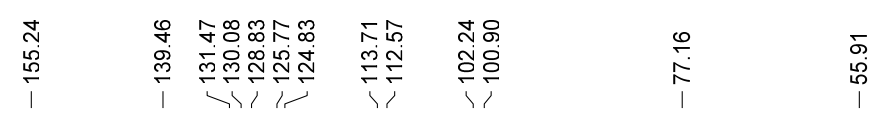
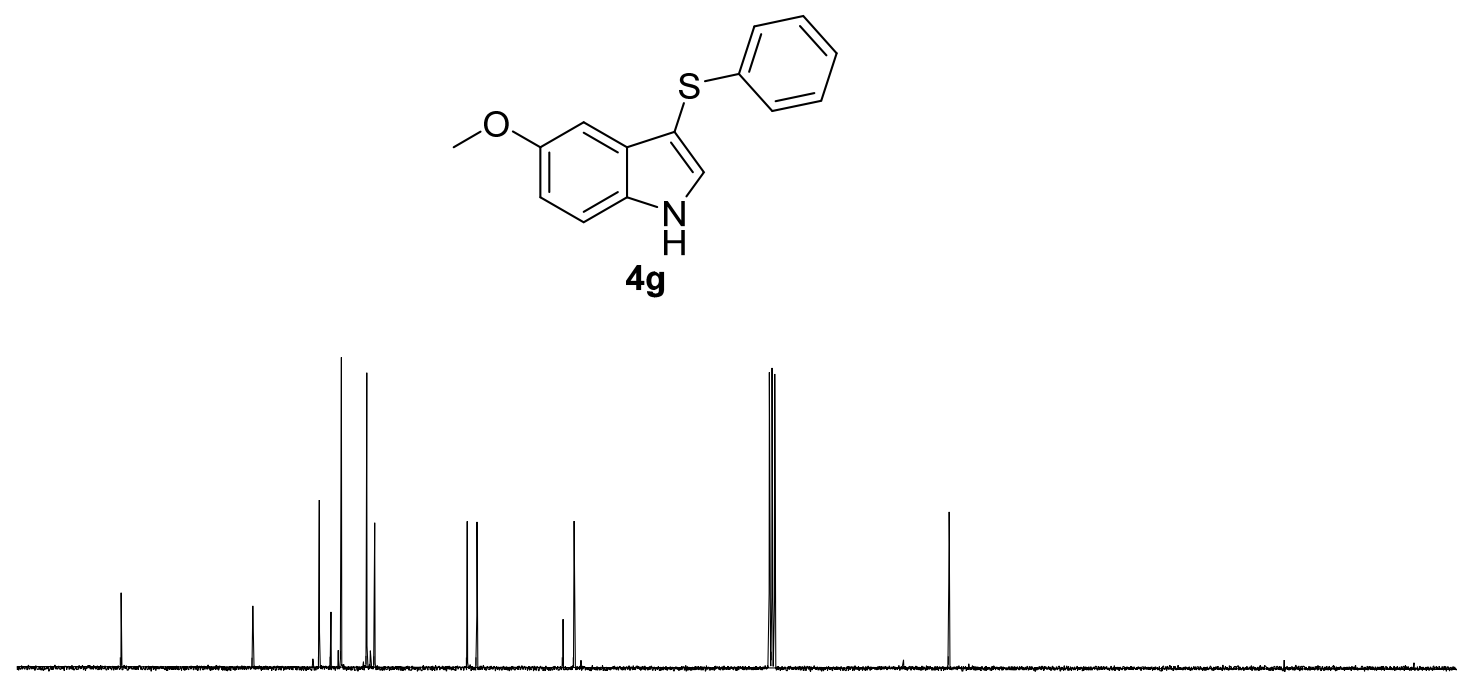

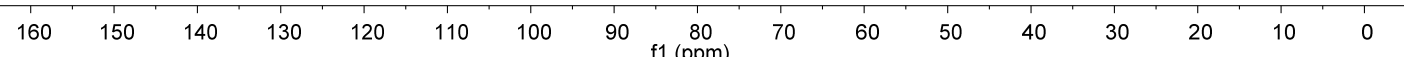

Figure S52 
${ }^{1} \mathrm{H}$ and ${ }^{13} \mathrm{C}$ NMR Spectrum of Compound $\mathbf{4 h}$

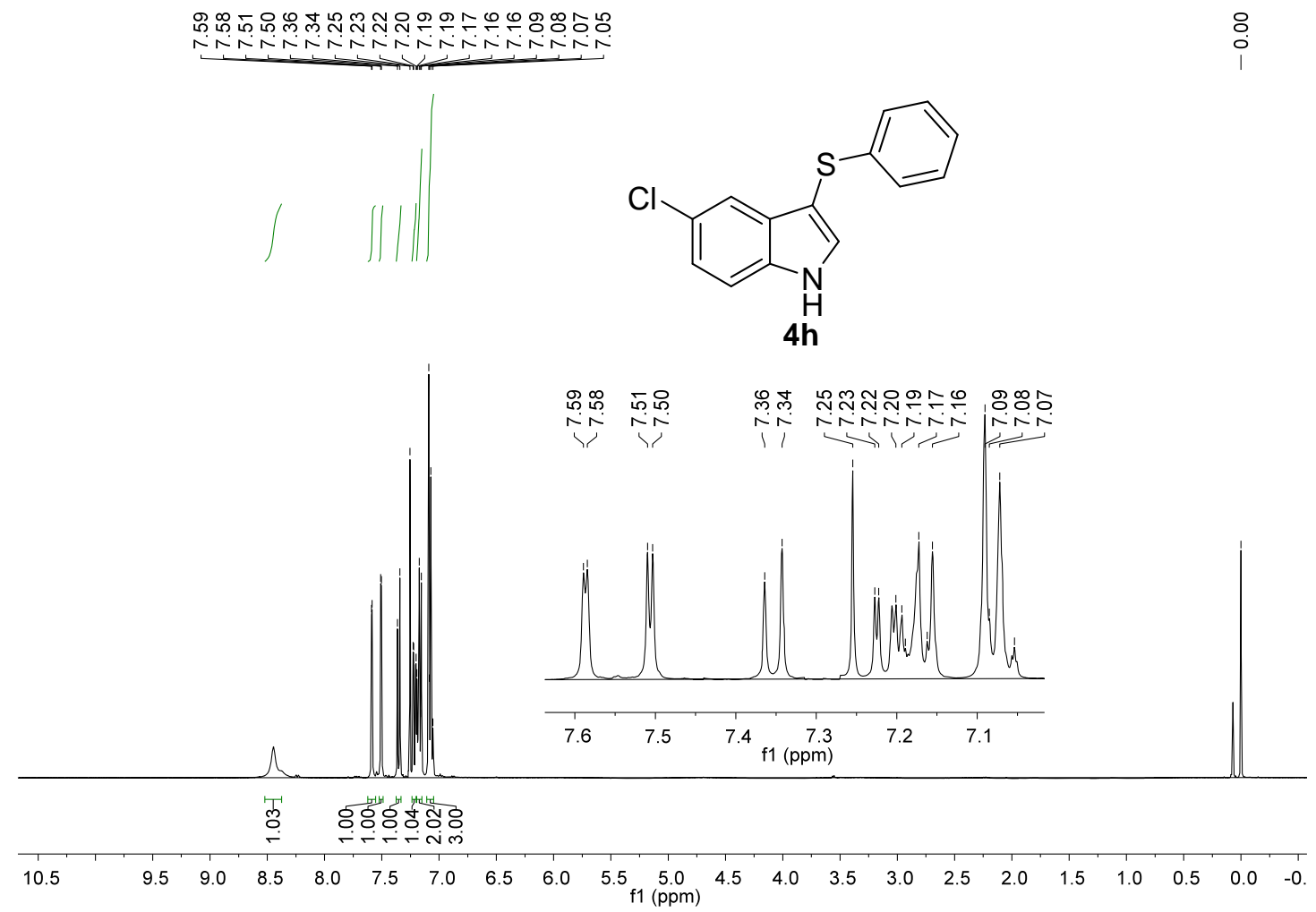

Figure S53

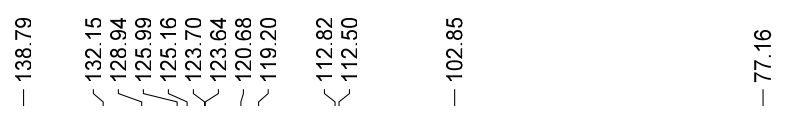

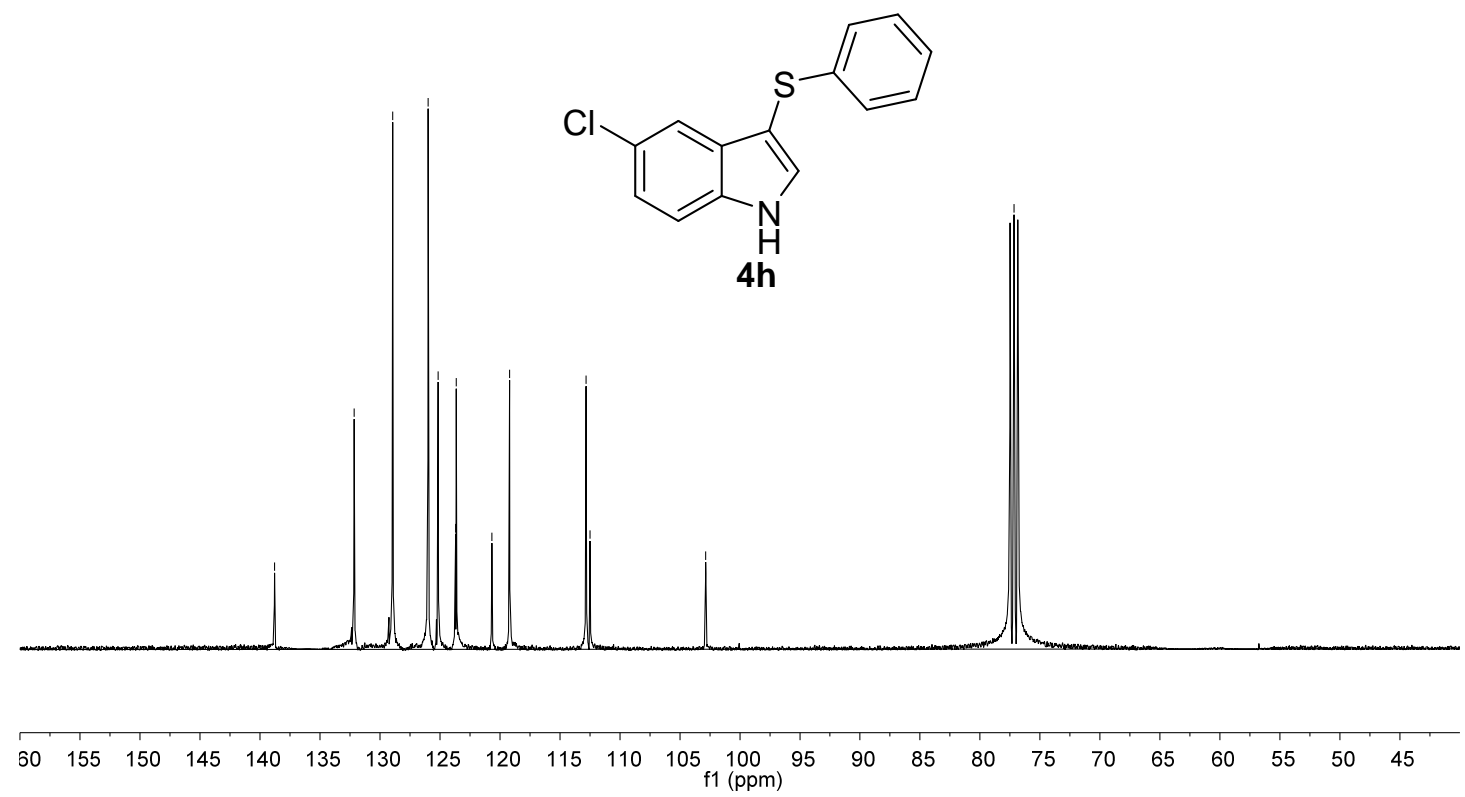

Figure S54 
${ }^{1} \mathrm{H},{ }^{13} \mathrm{C}$ NMR and ${ }^{19} \mathrm{~F}$ NMR Spectrum of Compound $\mathbf{4 i}$

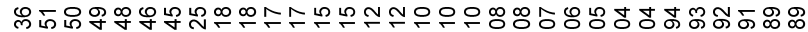

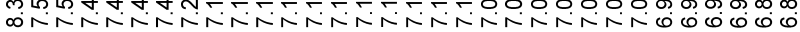

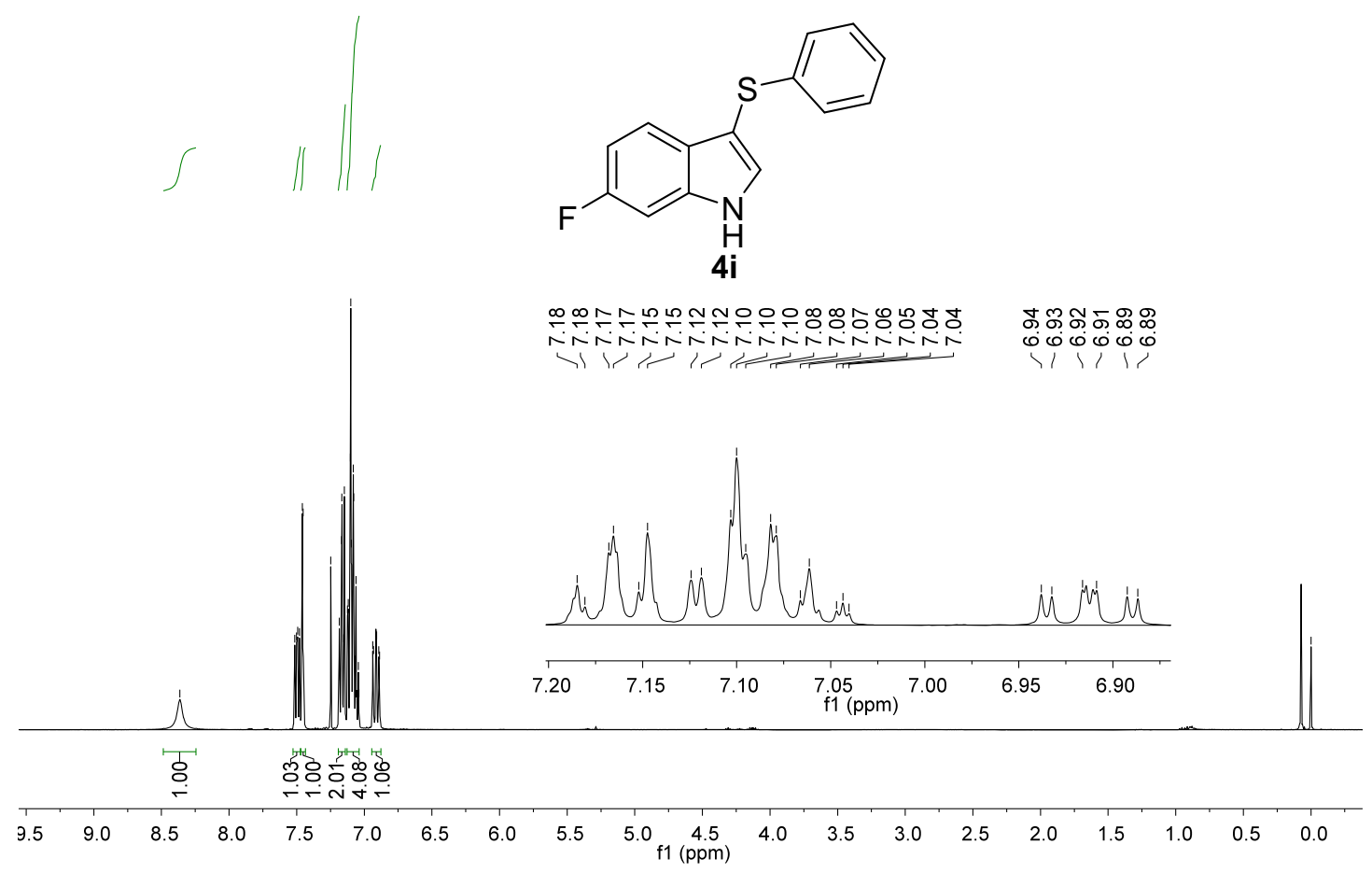

Figure S55

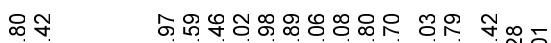

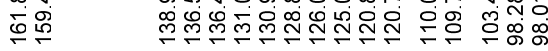

$\frac{0}{i}$

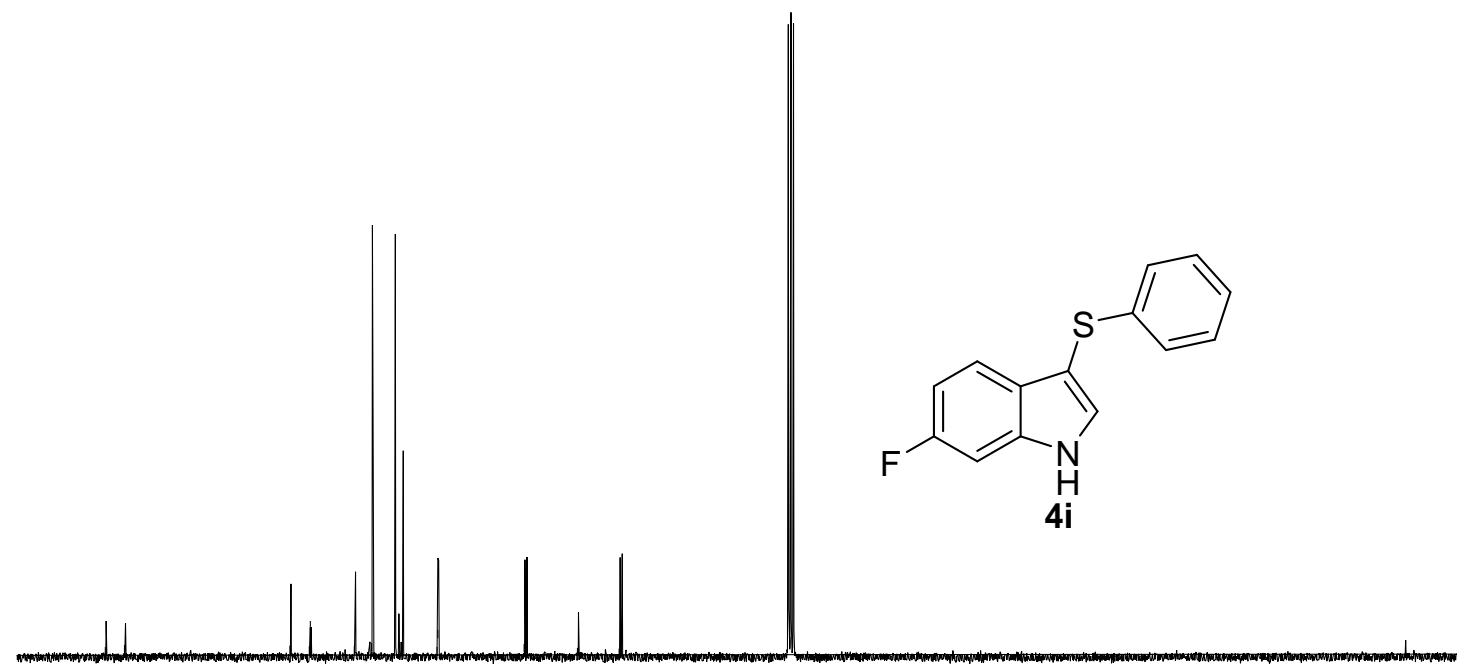

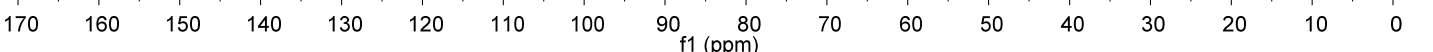

Figure S56 

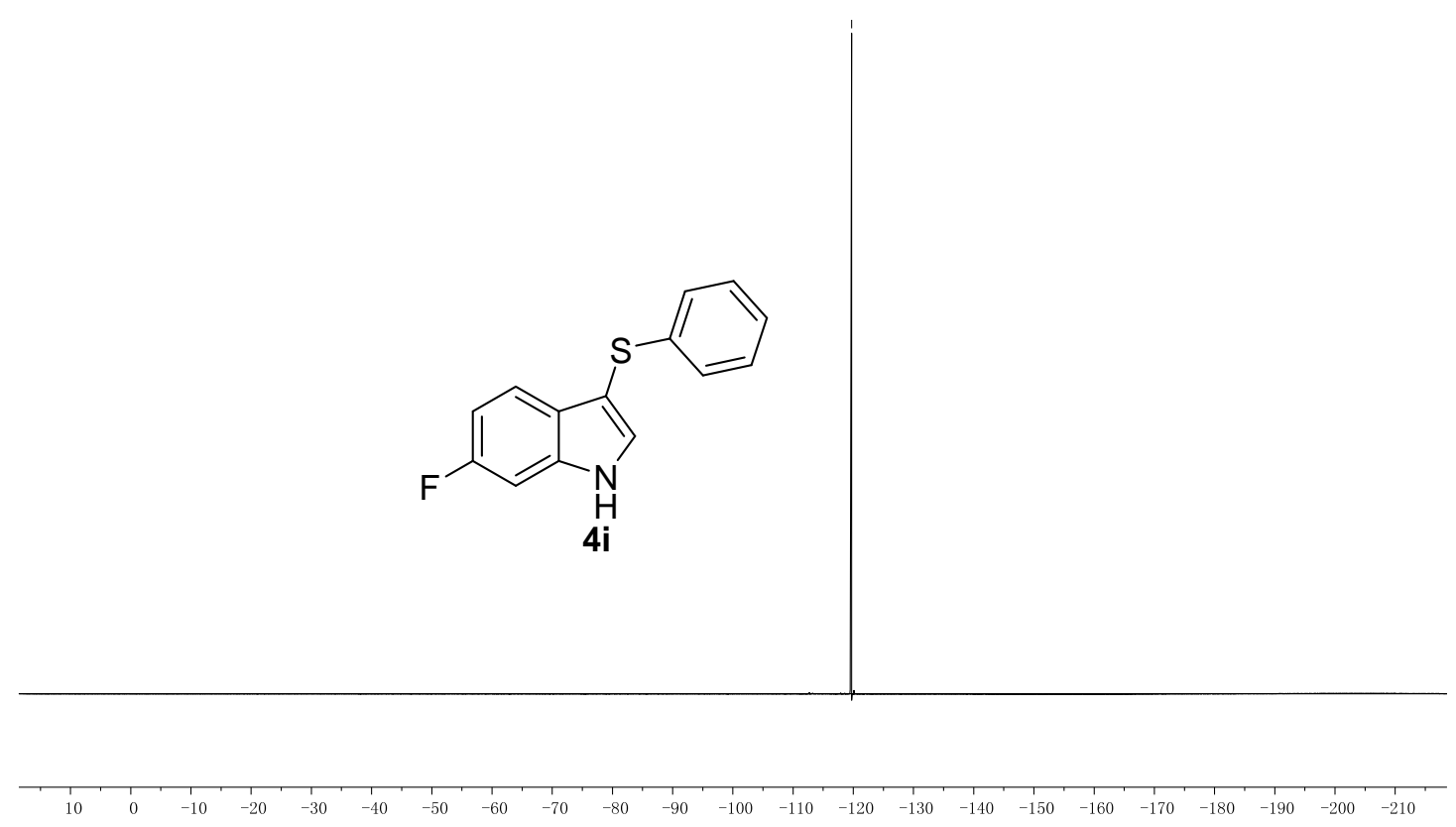

Figure S57

${ }^{1} \mathrm{H}$ and ${ }^{13} \mathrm{C}$ NMR Spectrum of Compound $\mathbf{4 j}$

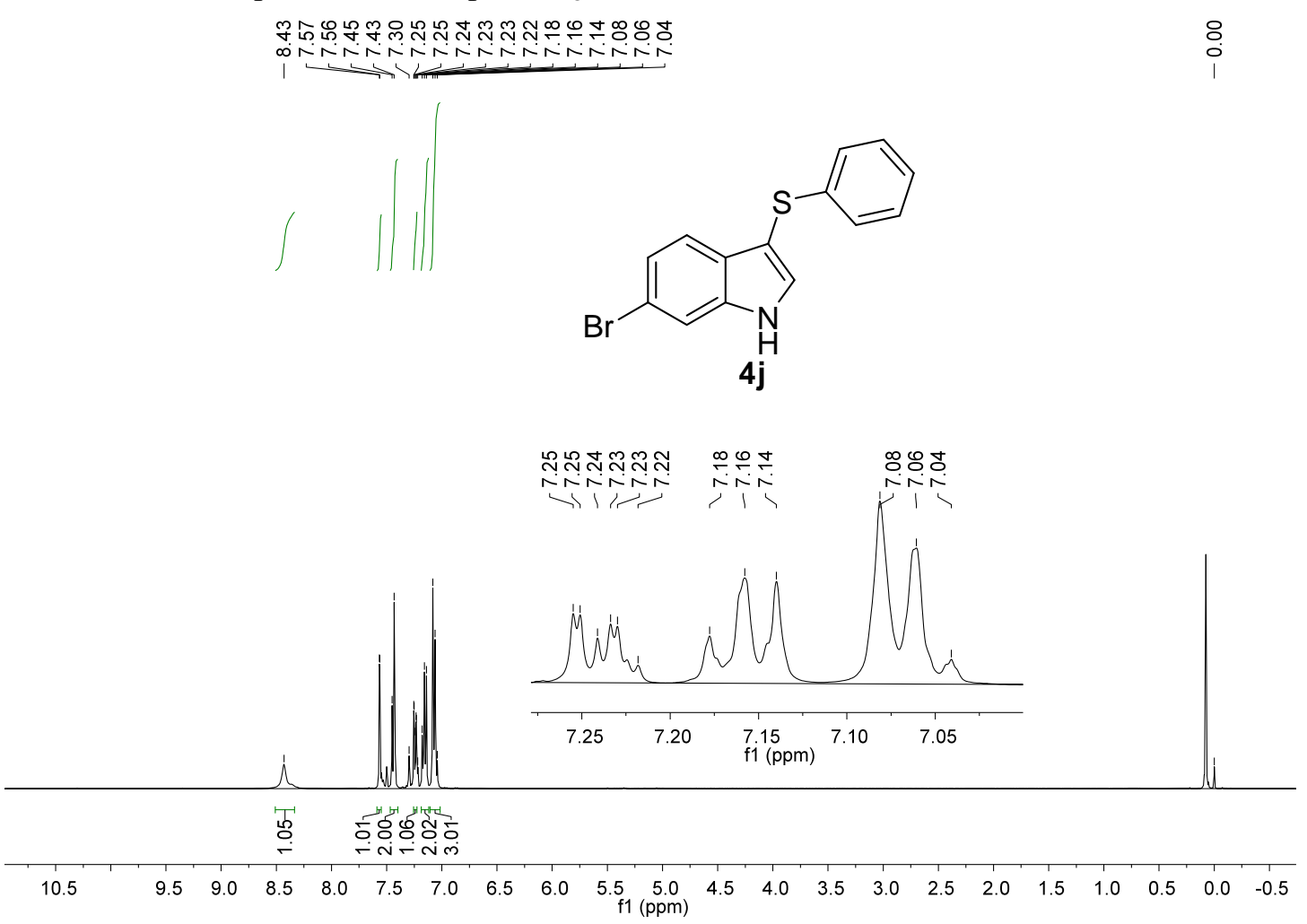

Figure S58 
il
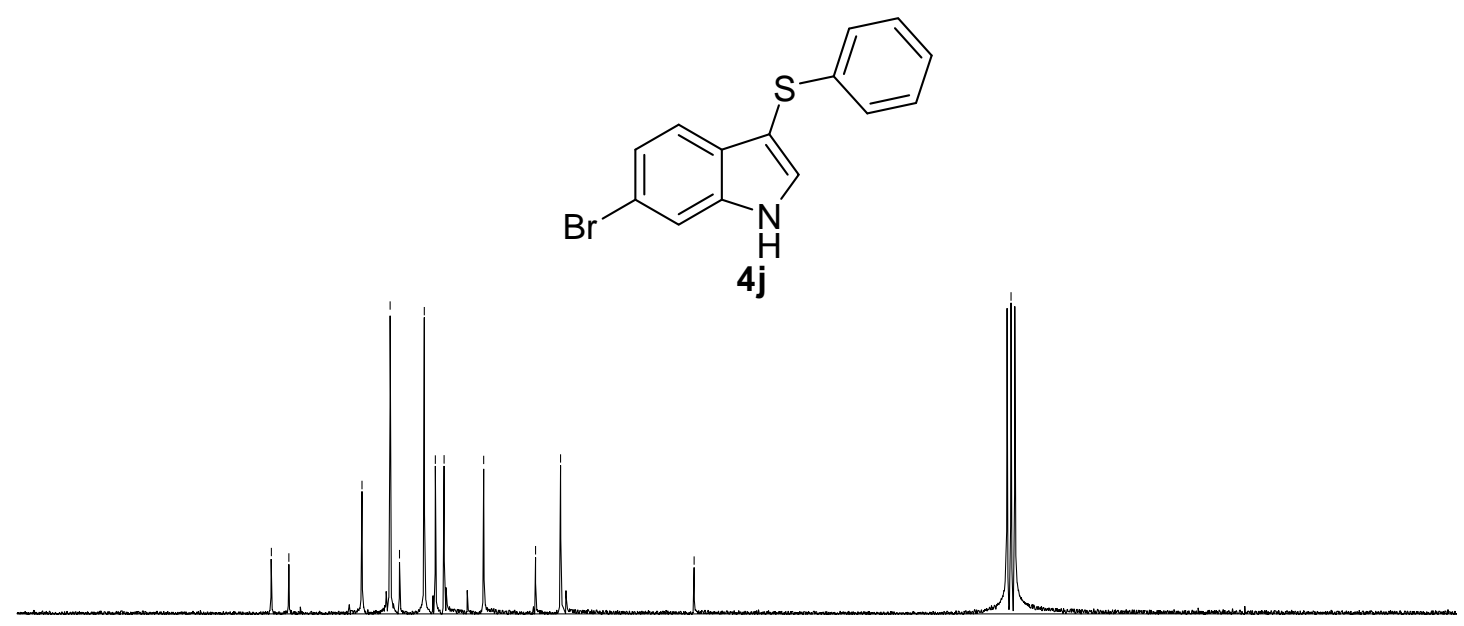

$\begin{array}{llllllllllllllllllllllll}60 & 155 & 150 & 145 & 140 & 135 & 130 & 125 & 120 & 115 & 110 & 105 & 100 & 95 & 90 & 85 & 80 & 75 & 70 & 65 & 60 & 55 & 50 & 45\end{array}$

Figure S59

${ }^{1} \mathrm{H}$ and ${ }^{13} \mathrm{C}$ NMR Spectrum of Compound $\mathbf{4 k}$

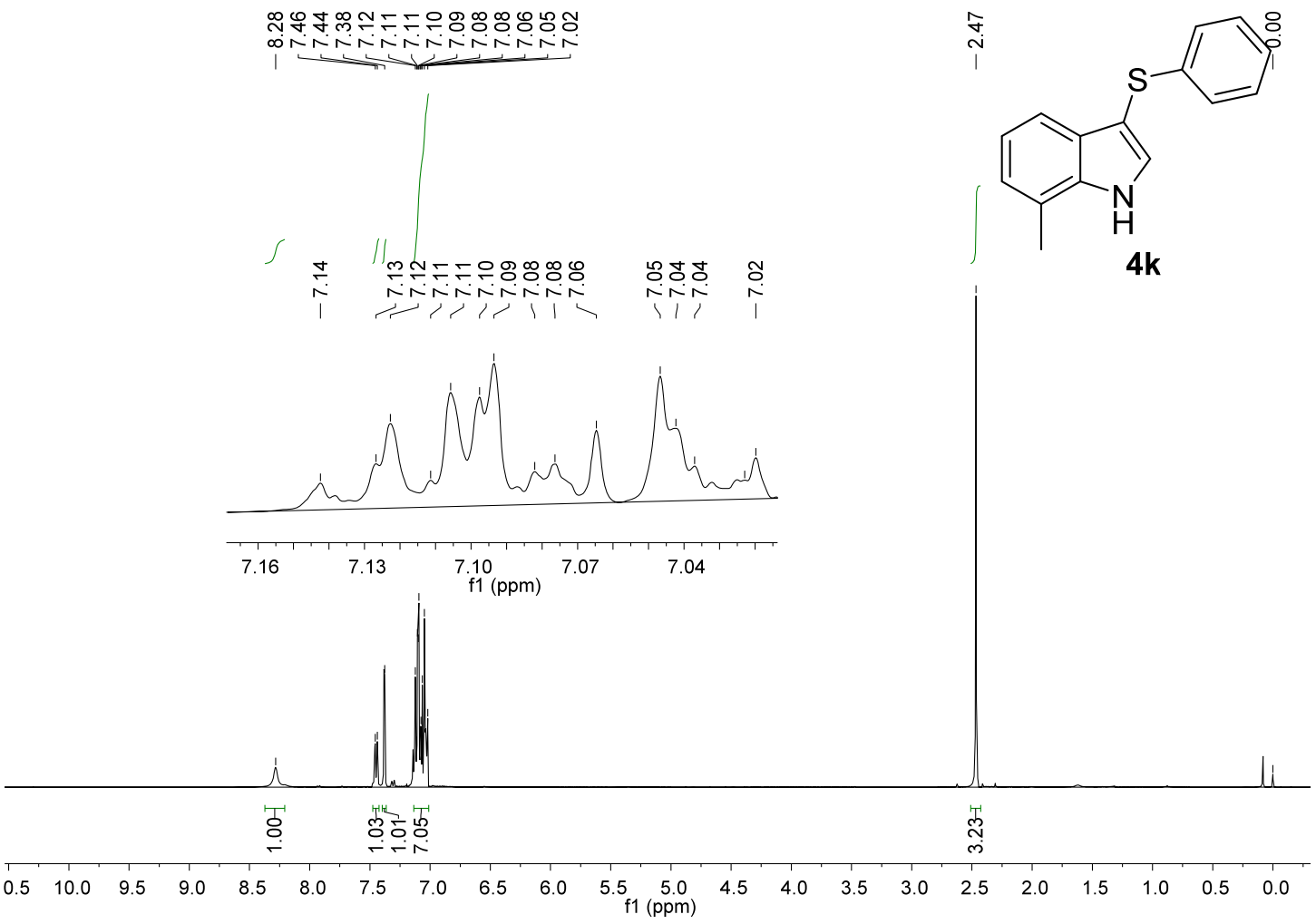

Figure S60 

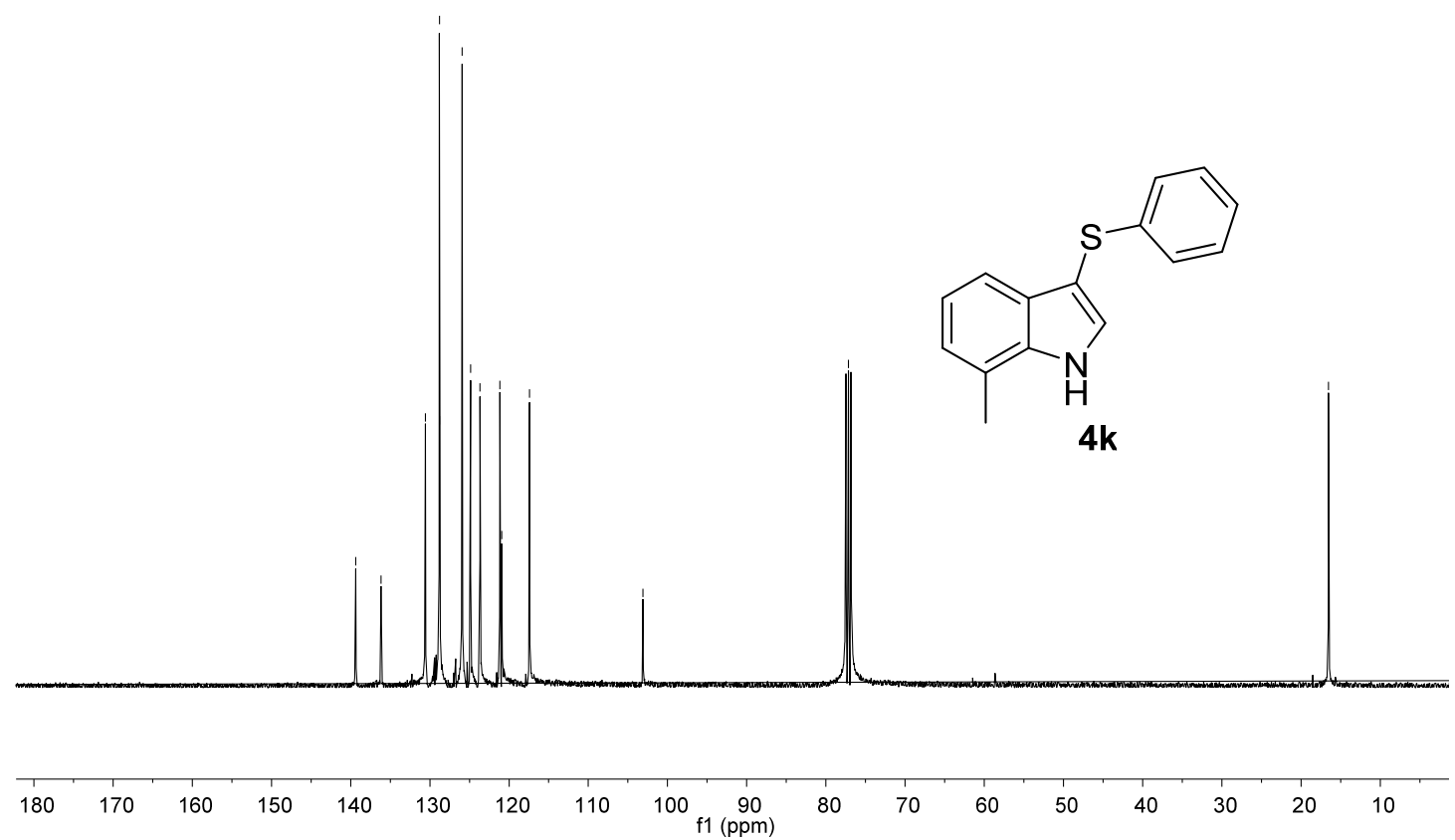

Figure S61

${ }^{1} \mathrm{H}$ and ${ }^{13} \mathrm{C}$ NMR Spectrum of Compound $4 \mathrm{l}$

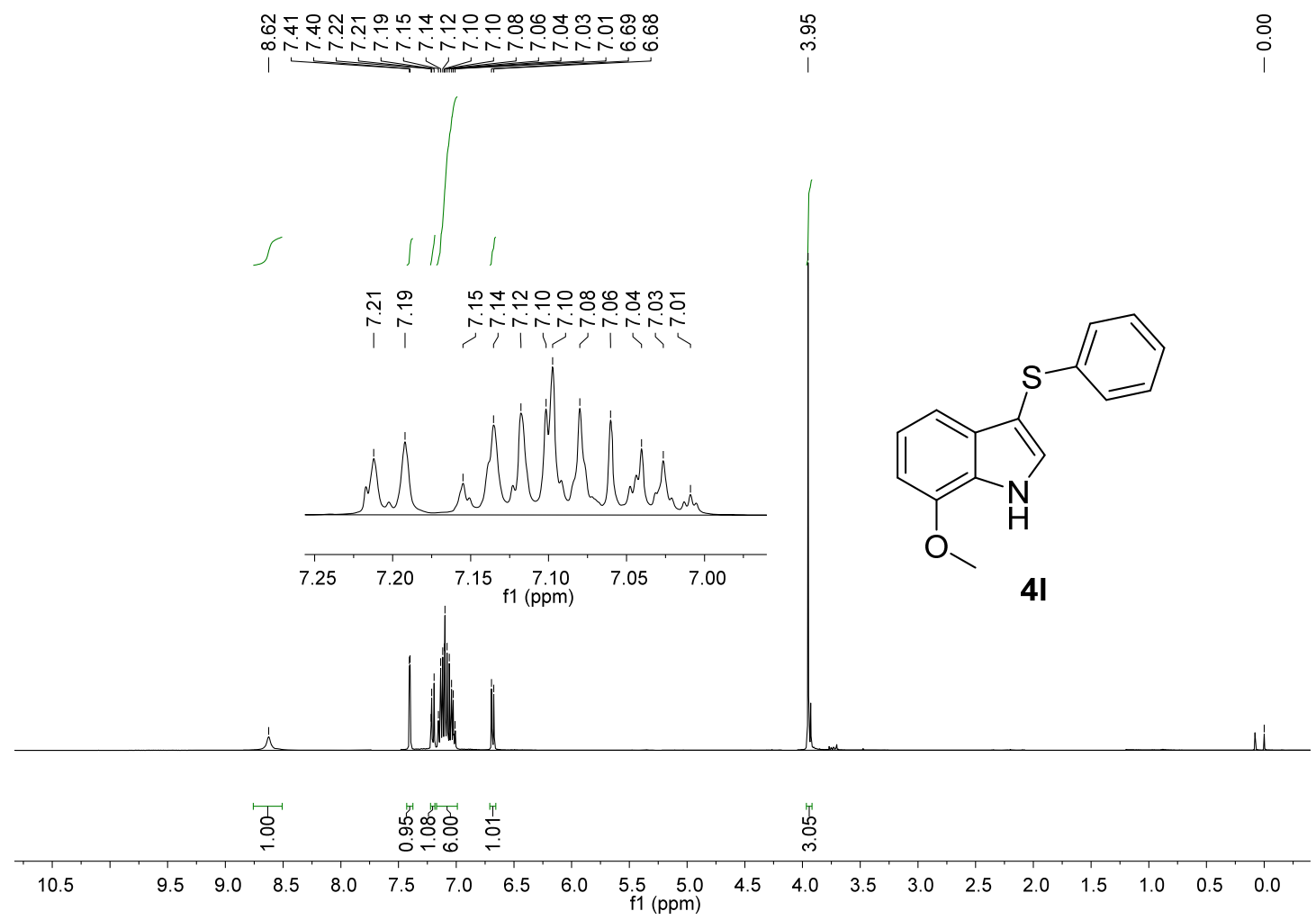

Figure S62 

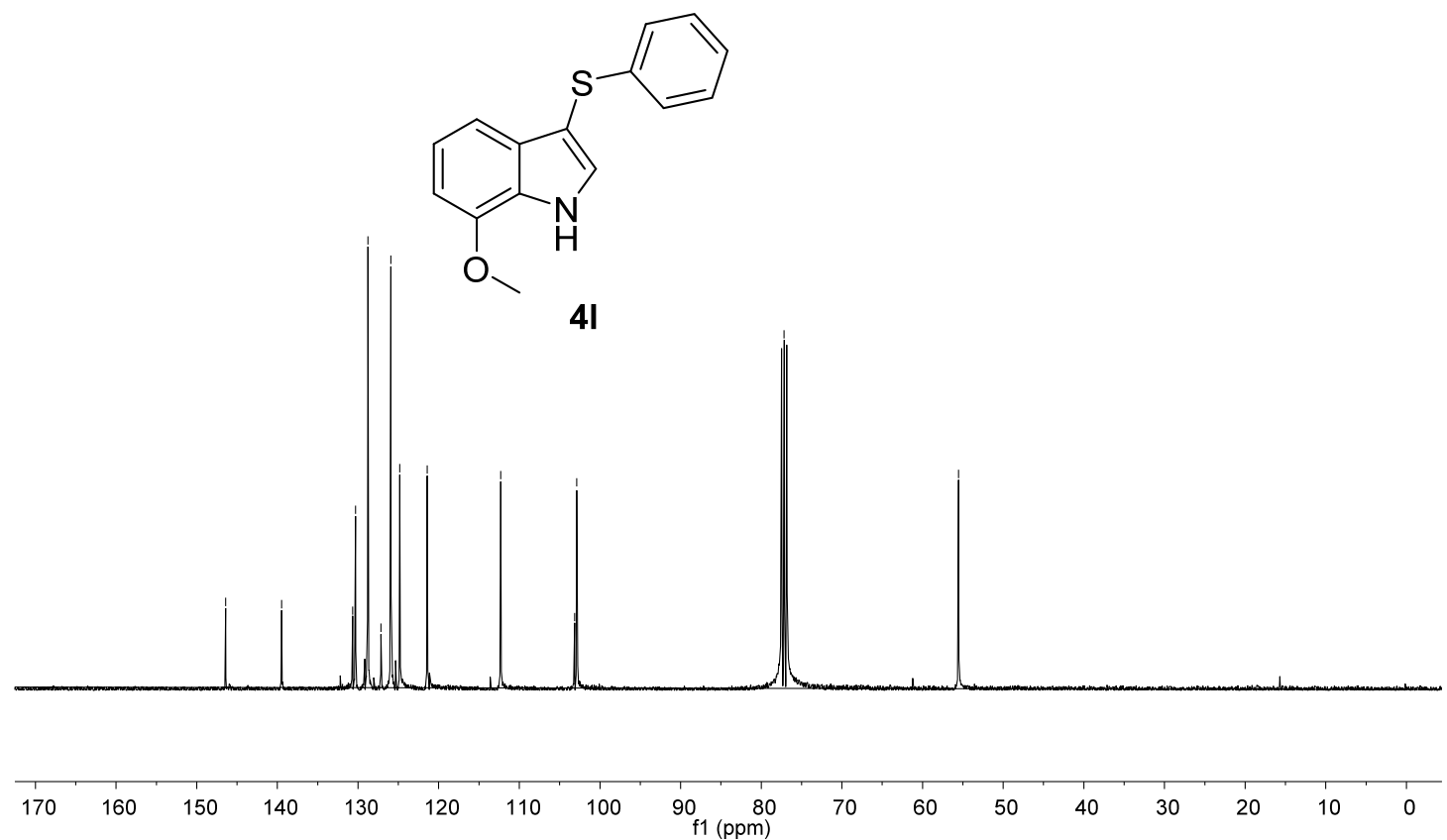

Figure S63

${ }^{1} \mathrm{H}$ and ${ }^{13} \mathrm{C}$ NMR Spectrum of Compound $\mathbf{4 m}$

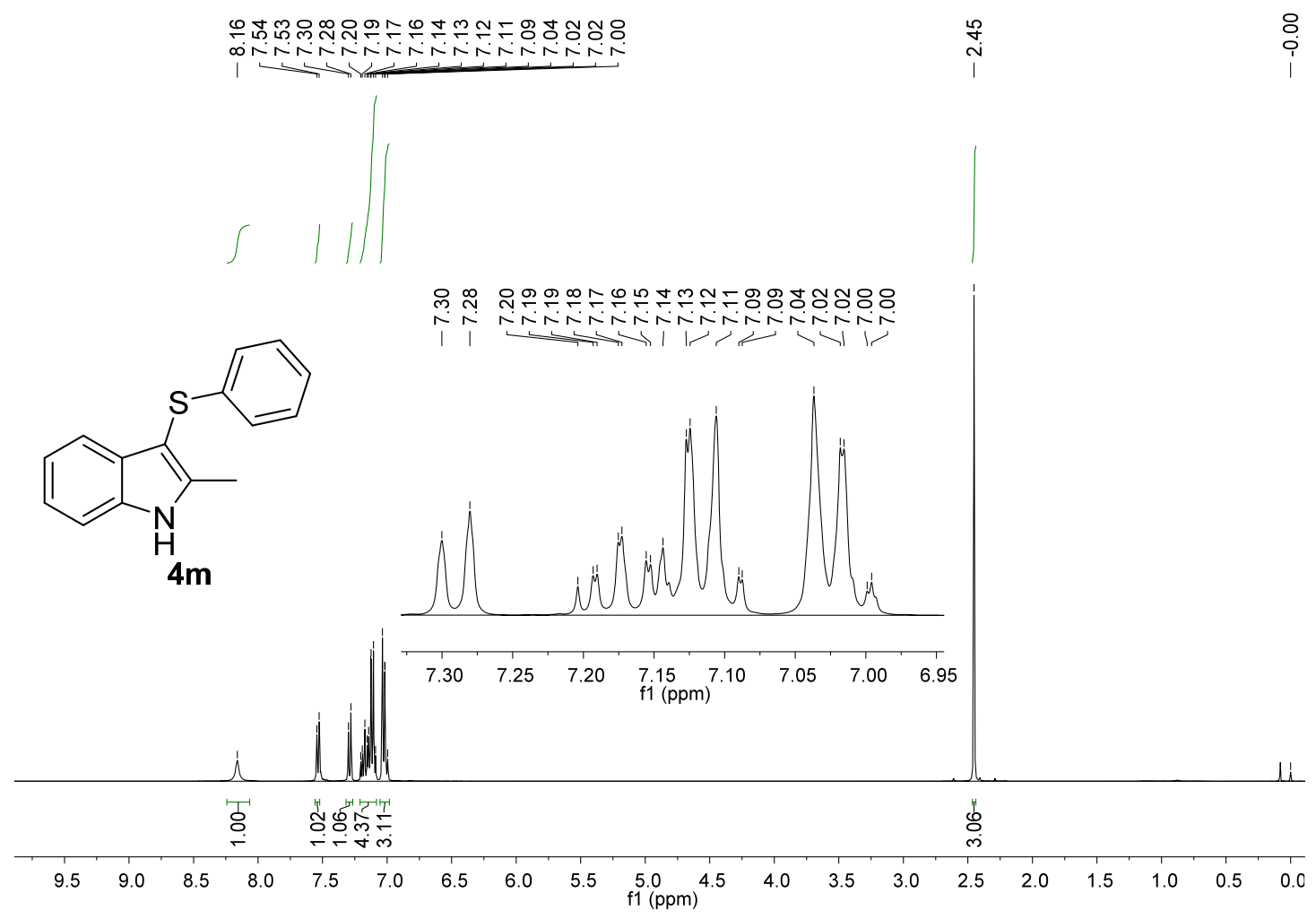

Figure S64 


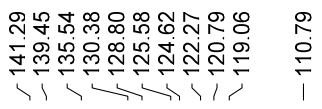

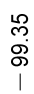

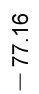

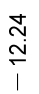

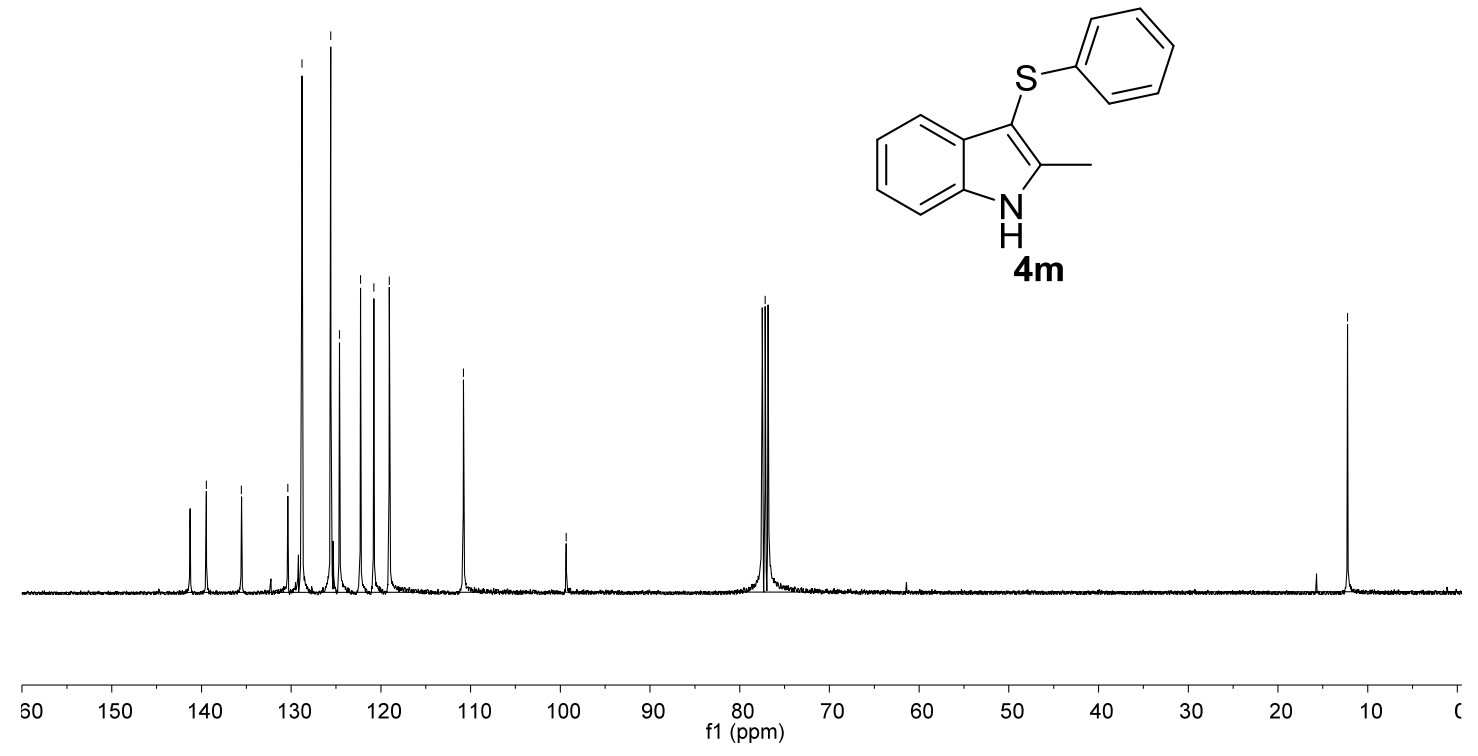

Figure S65

${ }^{1} \mathrm{H}$ and ${ }^{13} \mathrm{C}$ NMR Spectrum of Compound an

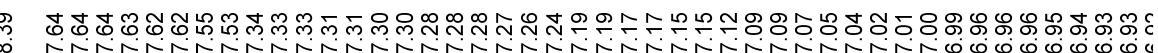

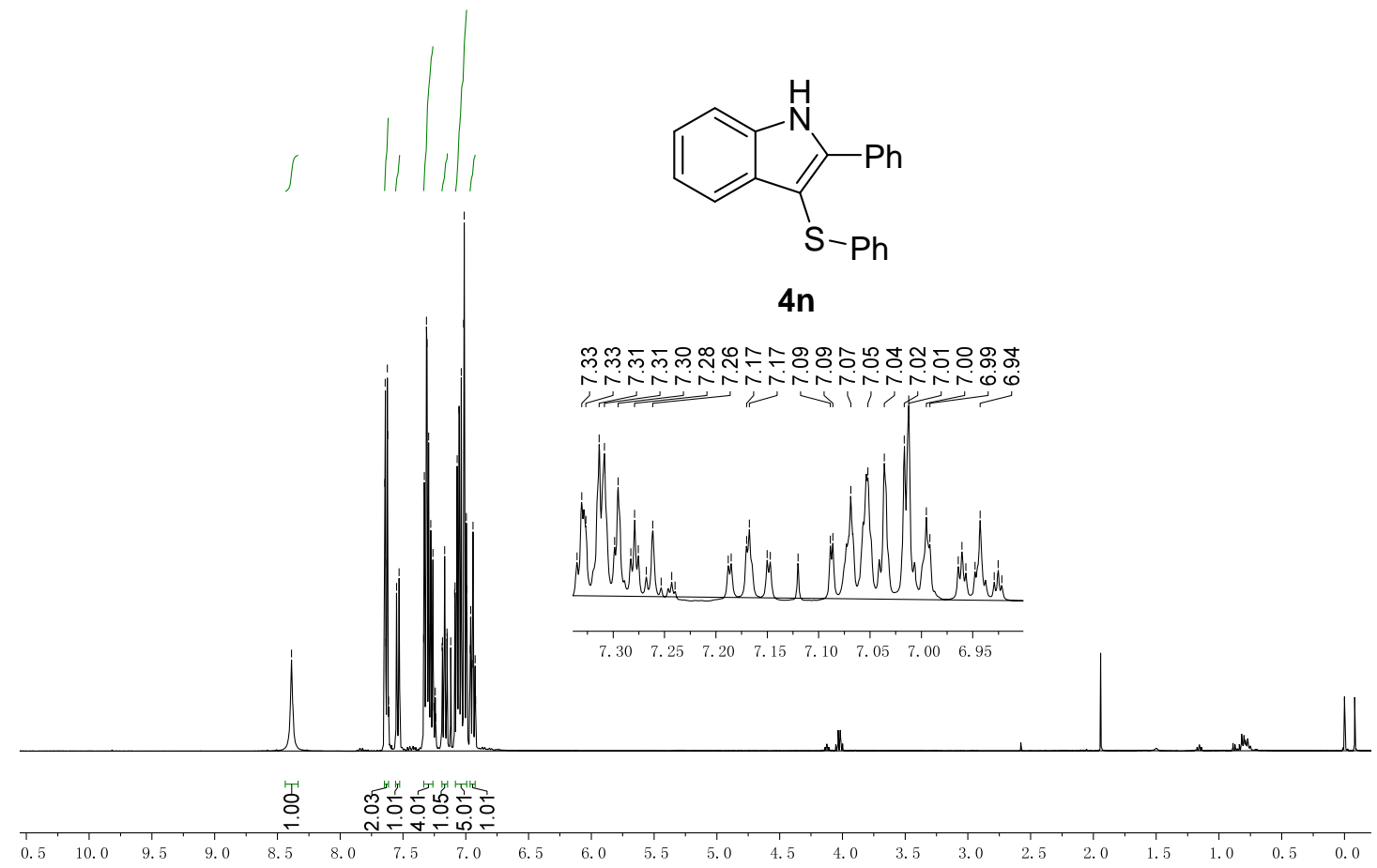

Figure S66

S34 


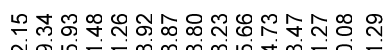

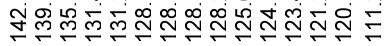

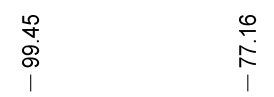

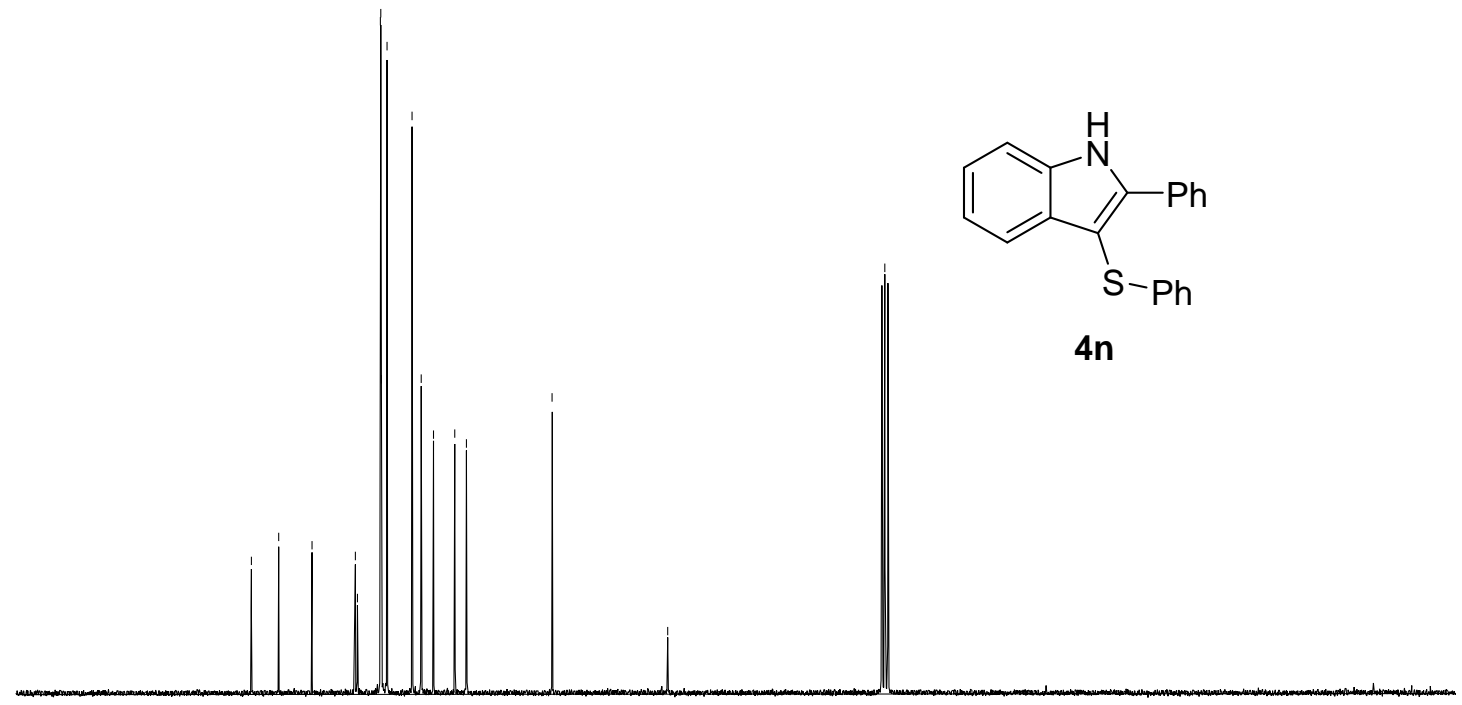

$\begin{array}{llllllllllllllllllllllllllllllll}165 & 160 & 155 & 150 & 145 & 140 & 135 & 130 & 125 & 120 & 115 & 110 & 105 & 100 & 95 & 90 & 85 & 80 & 75 & 70 & 65 & 60 & 55 & 50 & 45 & 40 & 35 & 30 & 25 & 20\end{array}$

Figure S67

${ }^{1} \mathrm{H}$ and ${ }^{13} \mathrm{C}$ NMR Spectrum of Compound 40

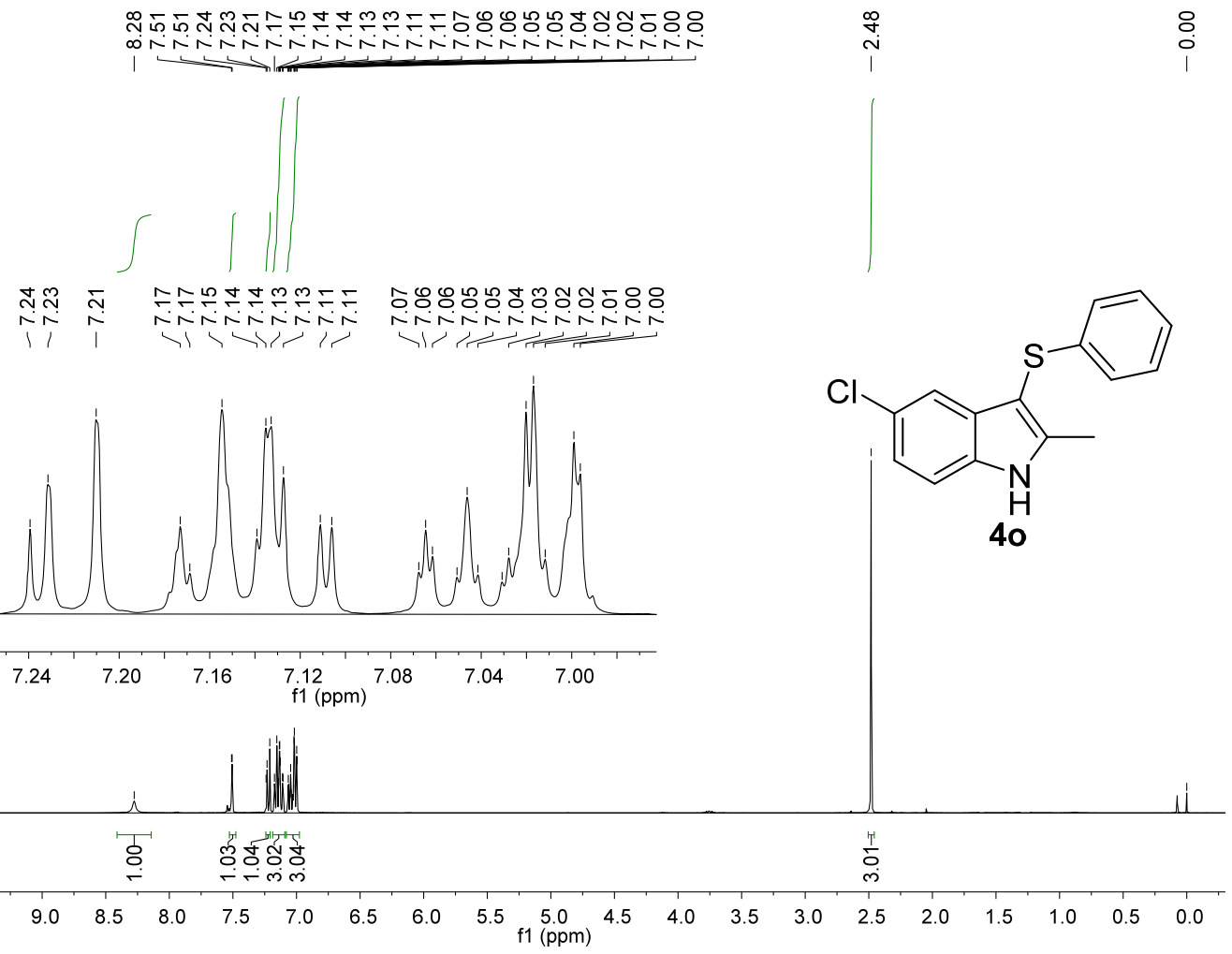

Figure S68 


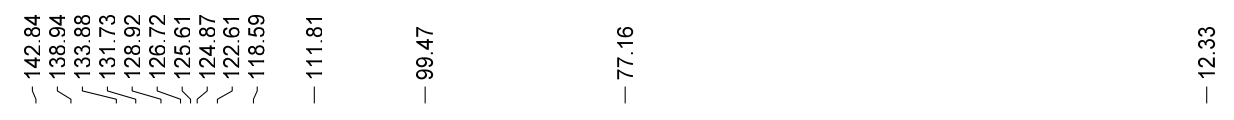
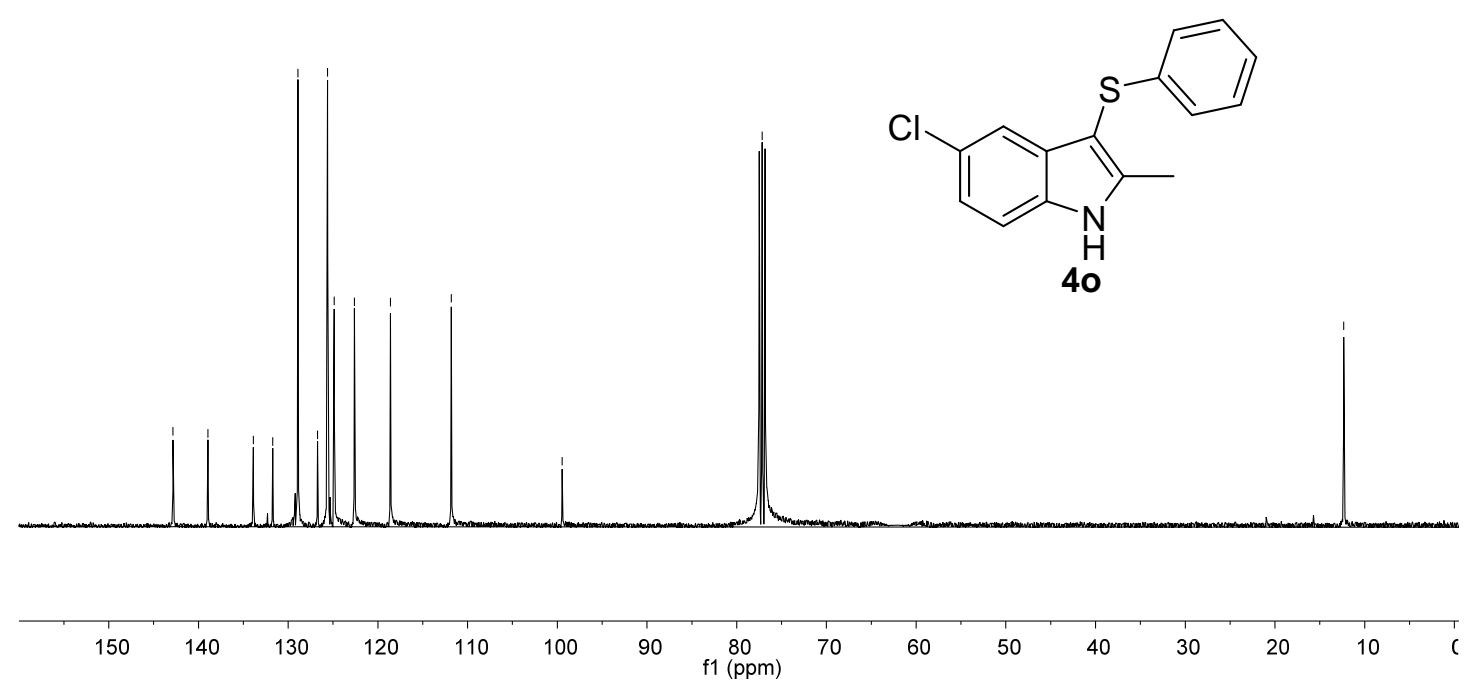

Figure S69

${ }^{1} \mathrm{H}$ and ${ }^{13} \mathrm{C}$ NMR Spectrum of Compound $\mathbf{4 p}$

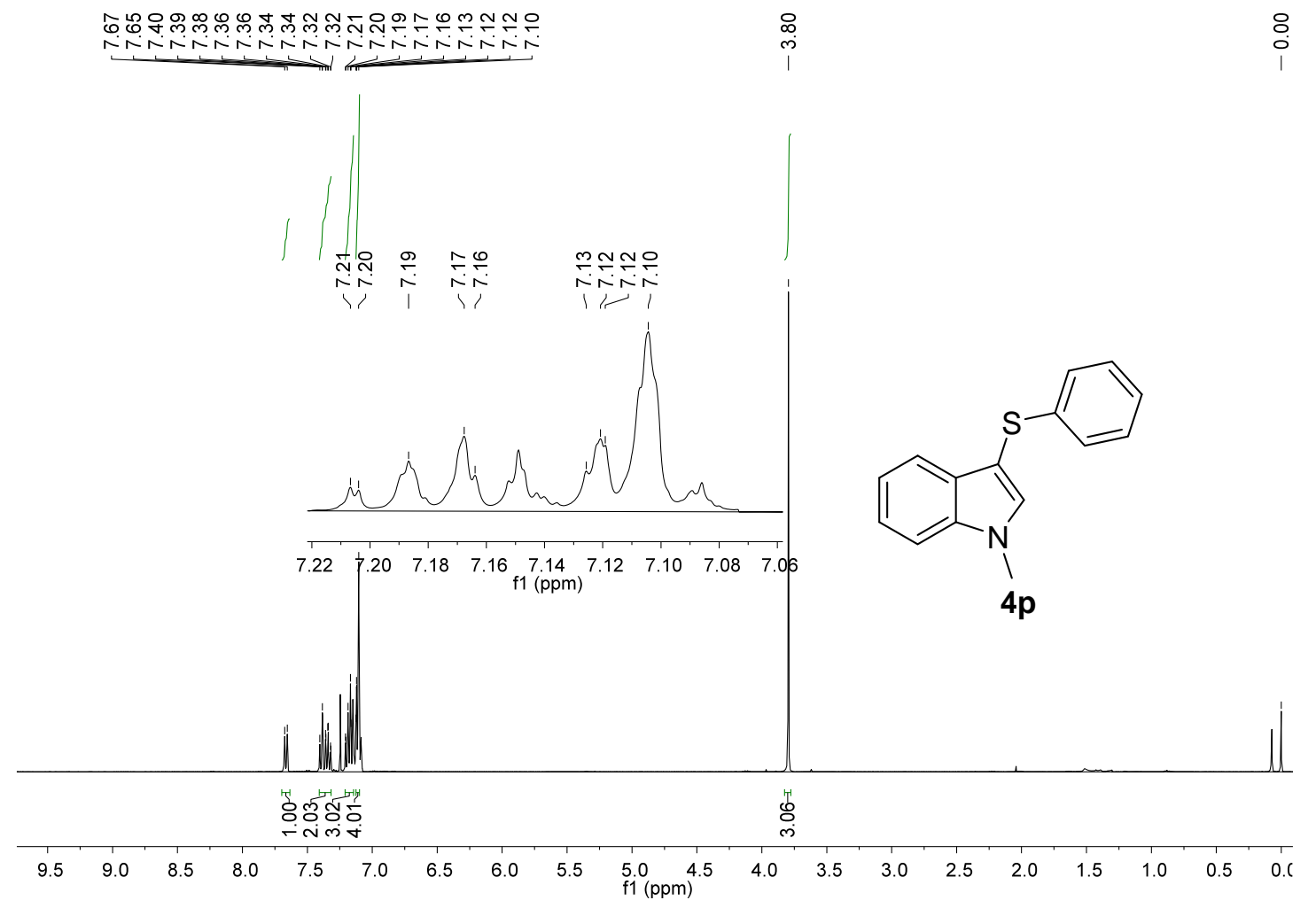

Figure S70 


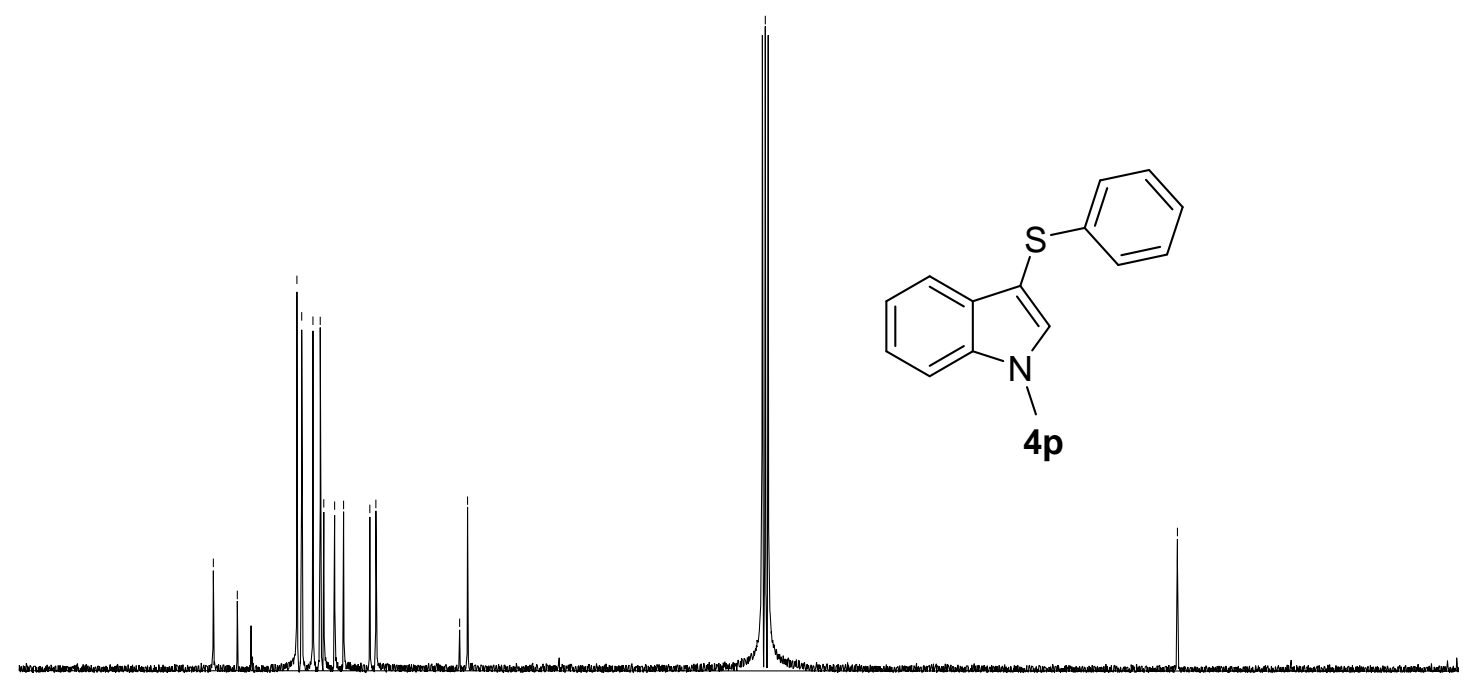

\begin{tabular}{lllllllllllllllll}
\hline 60 & 150 & 140 & 130 & 120 & 110 & 100 & 90 & $\begin{array}{c}80 \\
\mathrm{f} 1(\mathrm{ppm})\end{array}$ & 70 & 60 & 50 & 40 & 30 & 20 & 10 & 10
\end{tabular}

Figure S71

${ }^{1} \mathrm{H}$ and ${ }^{13} \mathrm{C}$ NMR Spectrum of Compound $\mathbf{4 q}$

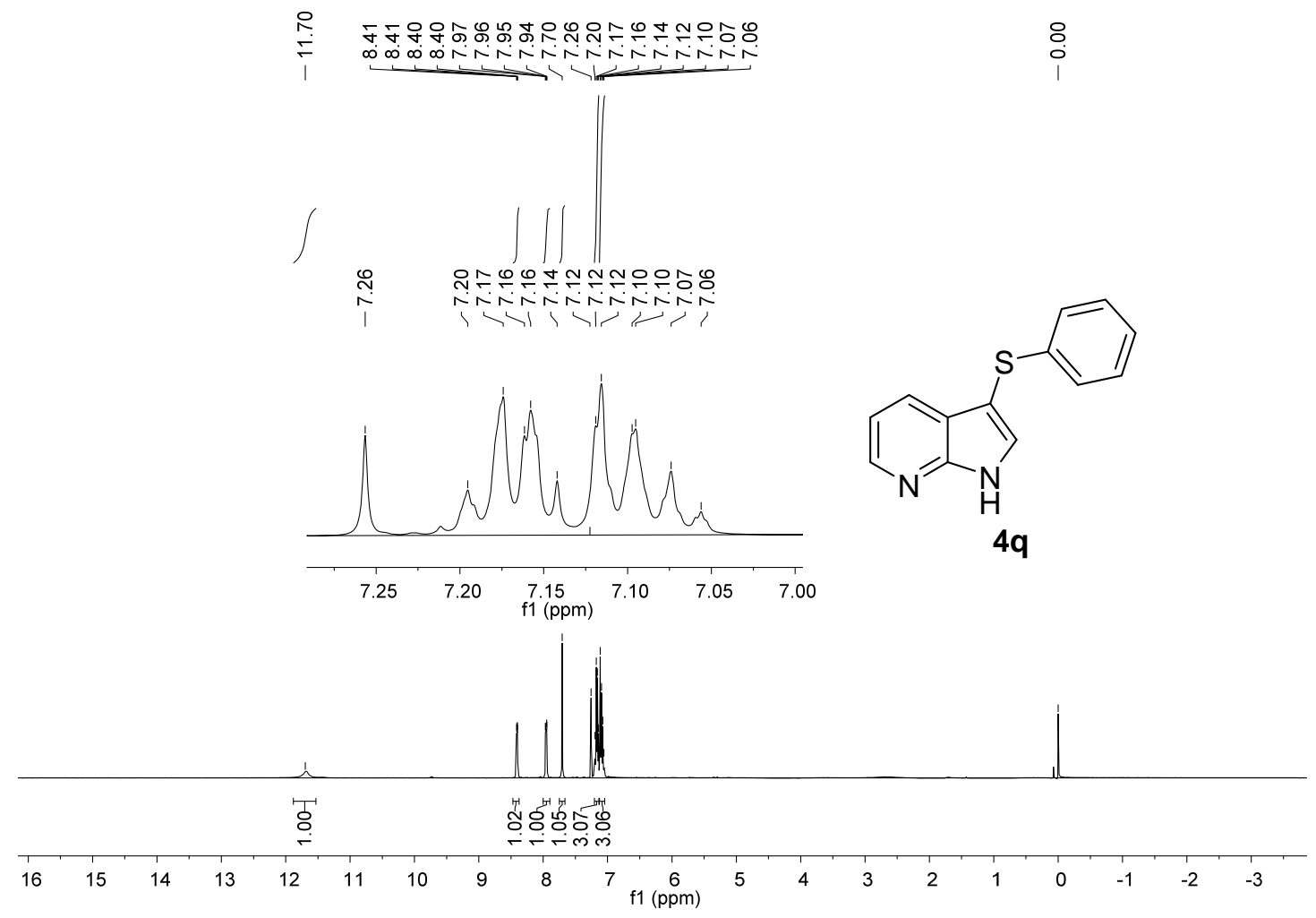

Figure S72 


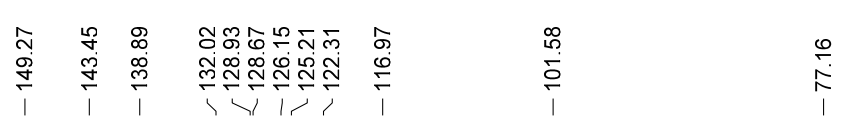

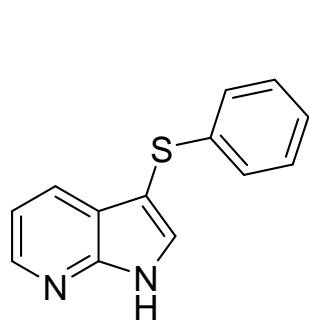

$4 q$

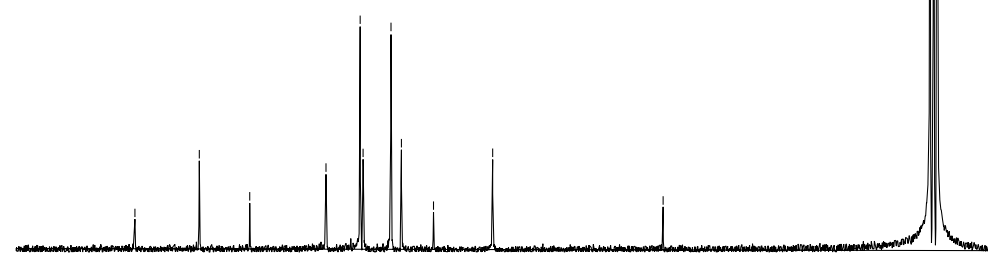

$\begin{array}{llllllllllllllllllll}155 & 145 & 135 & 125 & 115 & 105 & \begin{array}{c}95 \\ \mathrm{f} 1(\mathrm{ppm})\end{array} & 90 & 85 & 80 & 75 & 70 & 65 & 60 & 55 & 50 & 45 & 40 & 35\end{array}$

Figure S73

${ }^{1} \mathrm{H}$ and ${ }^{13} \mathrm{C}$ NMR Spectrum of Compound $4 \mathbf{r}$

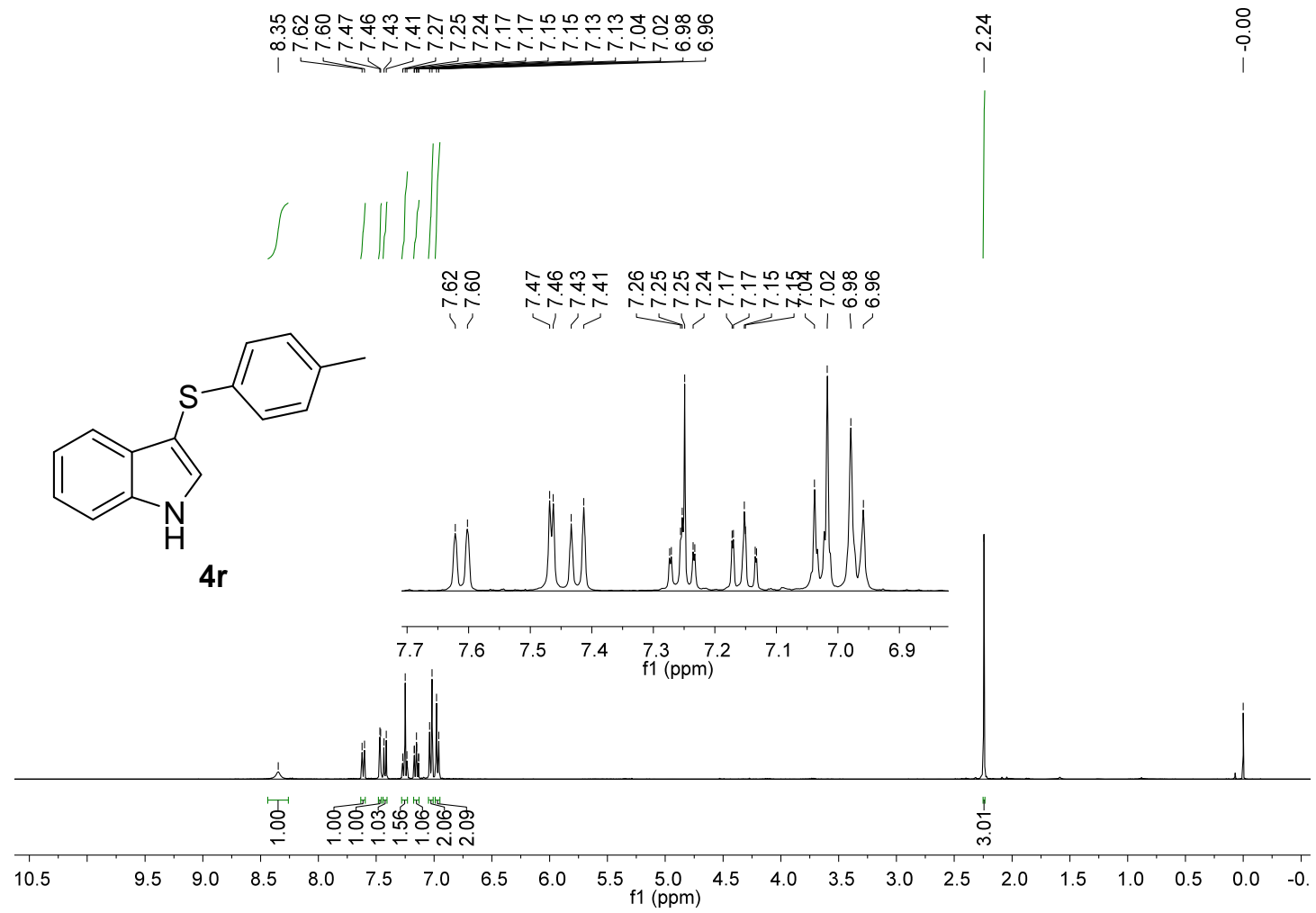

Figure S74 

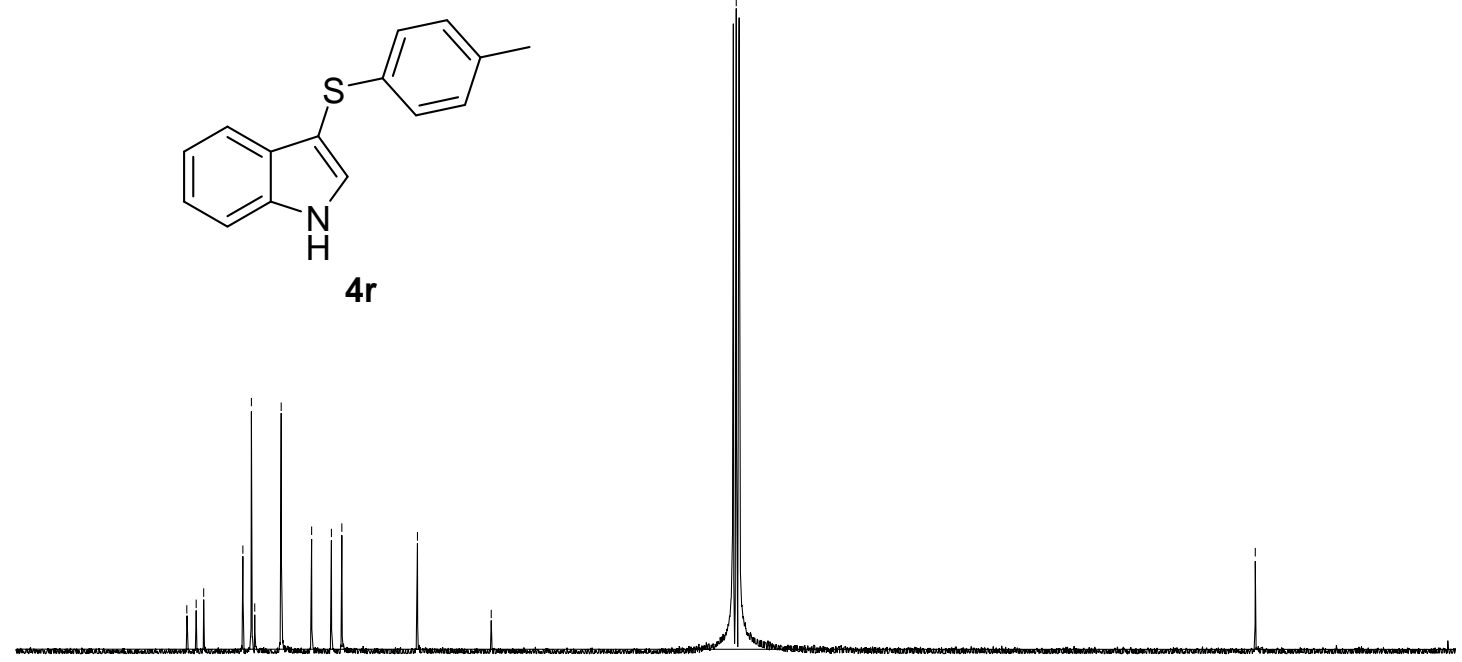

\begin{tabular}{|c|c|c|c|c|c|c|c|c|c|c|c|c|c|c|c|}
\hline 55 & 145 & 135 & 125 & 115 & 105 & 95 & 85 & $\begin{array}{c}75 \\
\mathrm{f} 1(\mathrm{ppm})\end{array}$ & 65 & 55 & 45 & 35 & 25 & 15 & 5 \\
\hline
\end{tabular}

\section{Figure S75}

${ }^{1} \mathrm{H}$ and ${ }^{13} \mathrm{C}$ NMR Spectrum of Compound $4 \mathbf{s}$

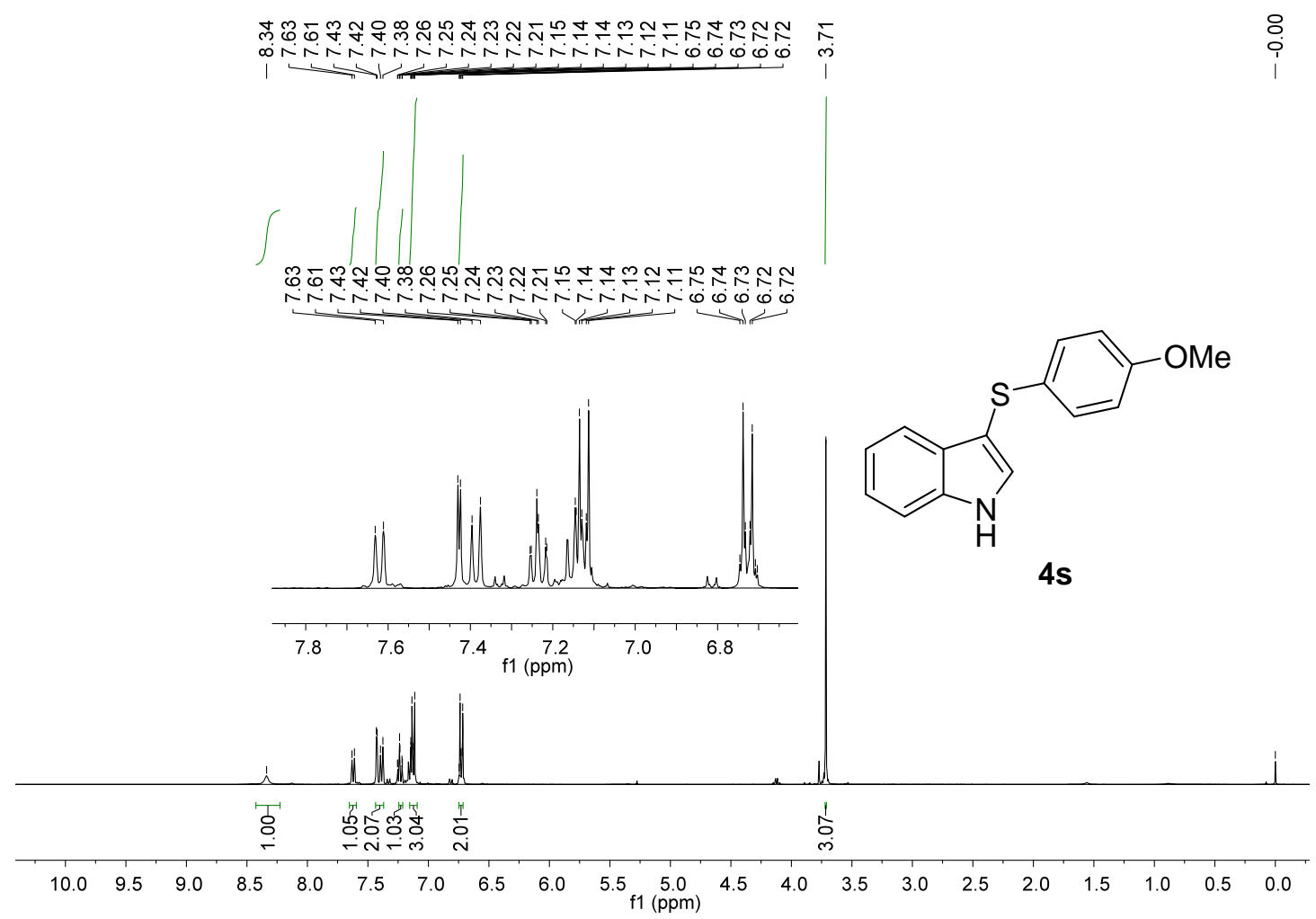

Figure S76 
<smiles>COc1ccc(Sc2c[nH]c3ccccc23)cc1</smiles>

$4 s$

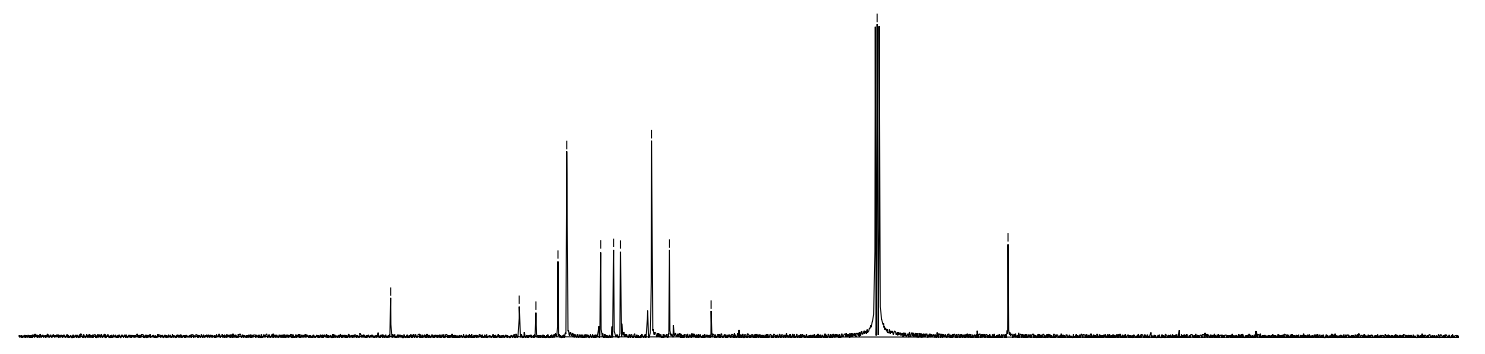

$\begin{array}{llllllllllllllllllllllll}210 & 200 & 190 & 180 & 170 & 160 & 150 & 140 & 130 & 120 & 110_{\mathrm{f} 1(\mathrm{ppm})}^{100} & 90 & 80 & 70 & 60 & 50 & 40 & 30 & 20 & 10 & 0 & -10\end{array}$

Figure S77

${ }^{1} \mathrm{H}$ and ${ }^{13} \mathrm{C}$ NMR Spectrum of Compound $\mathbf{4 t}$

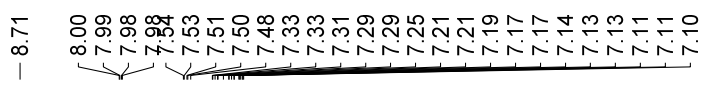
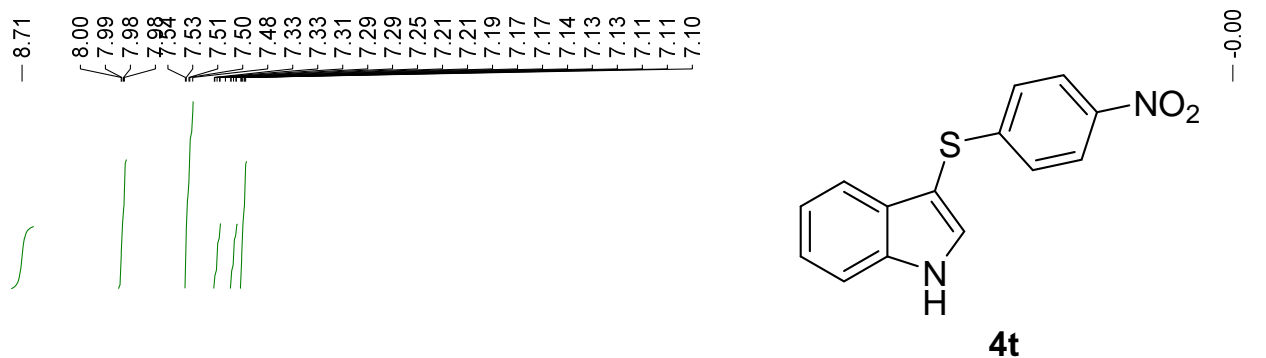

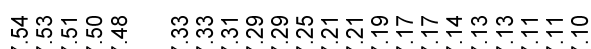

$4 t$

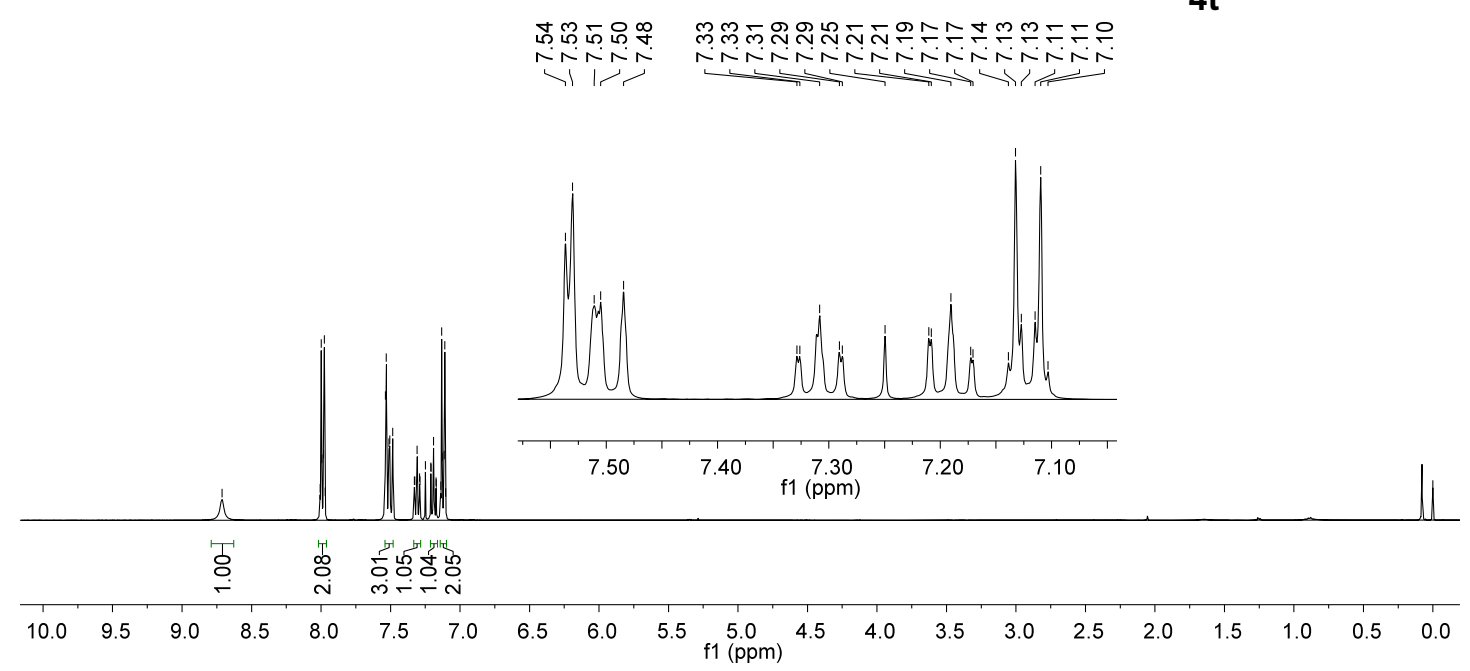

Figure S78 


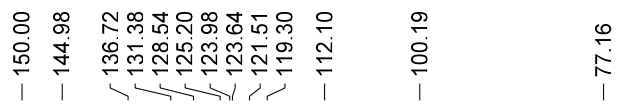
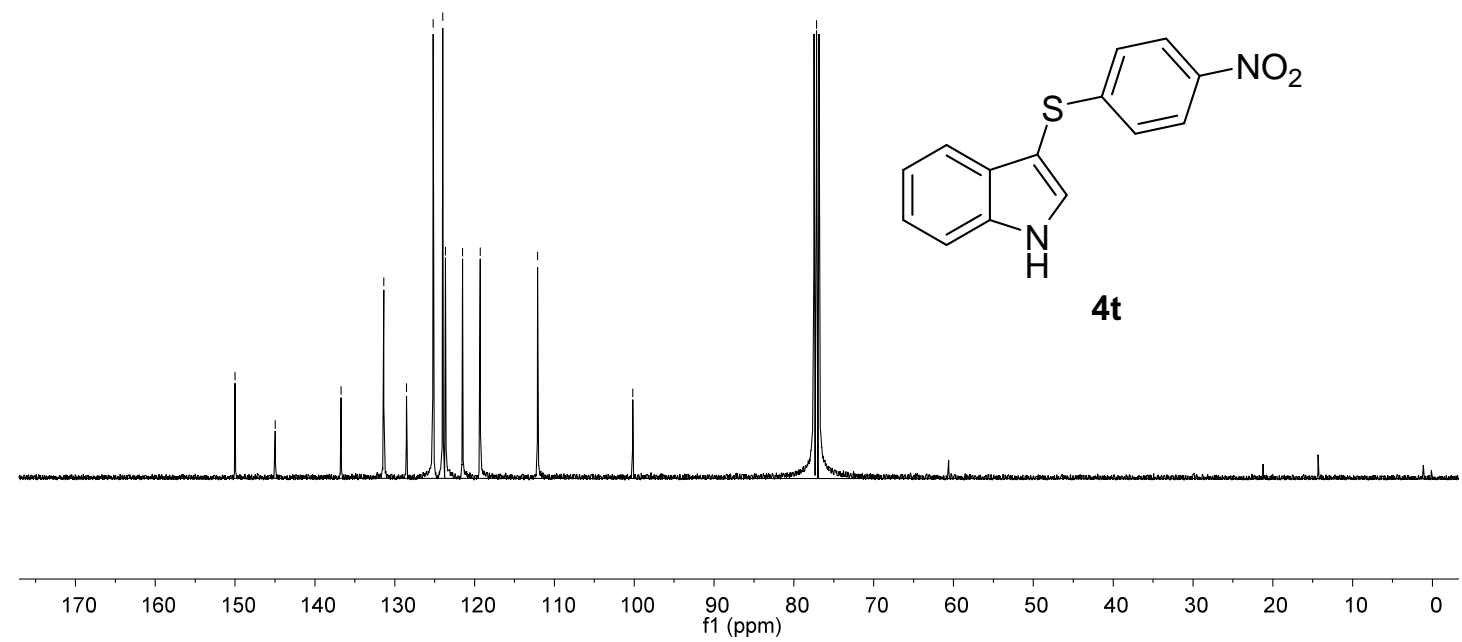

Figure S79

${ }^{1} \mathrm{H}$ and ${ }^{13} \mathrm{C}$ NMR Spectrum of Compound 5a

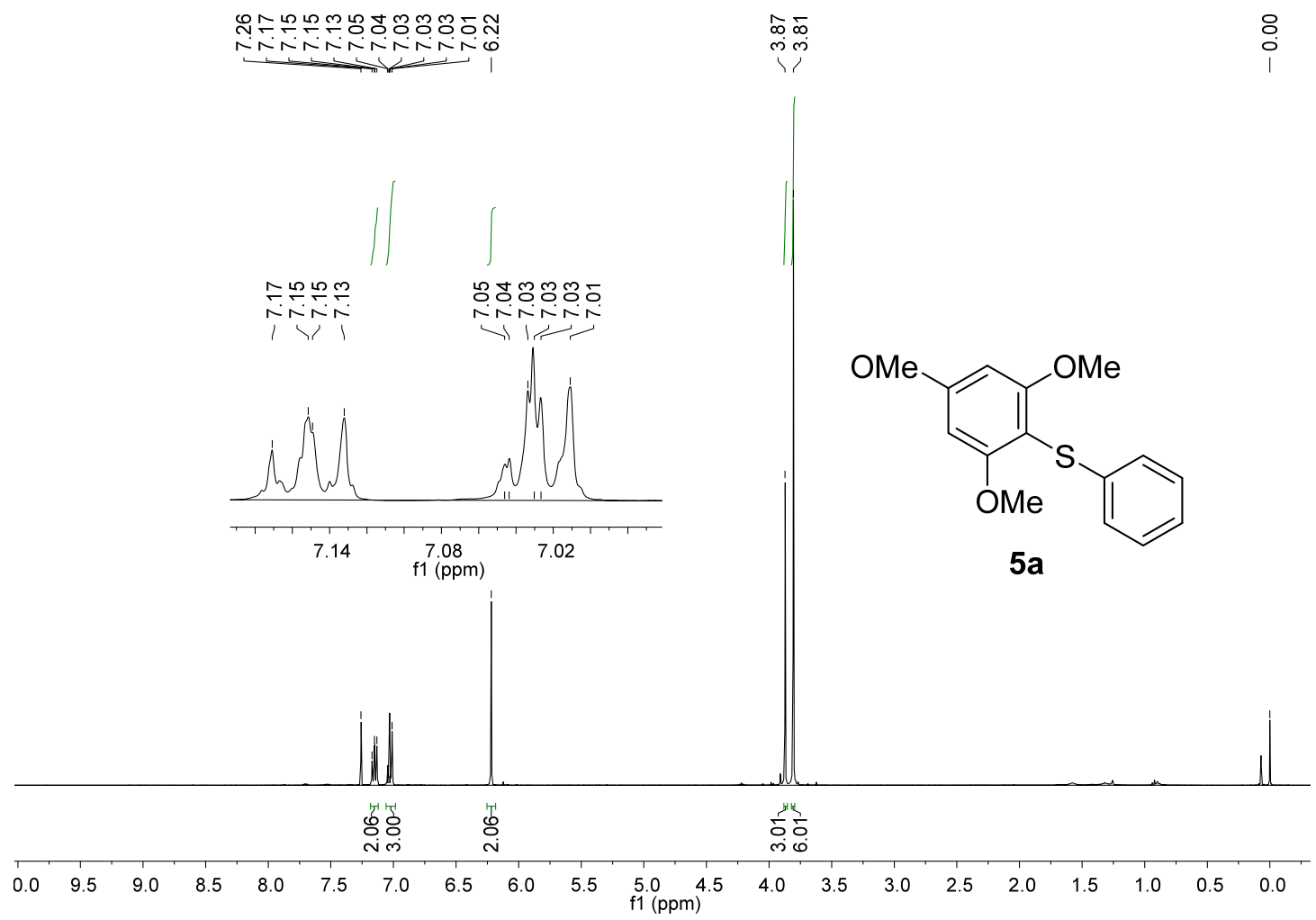

Figure S80 


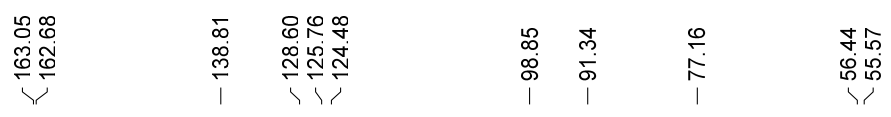
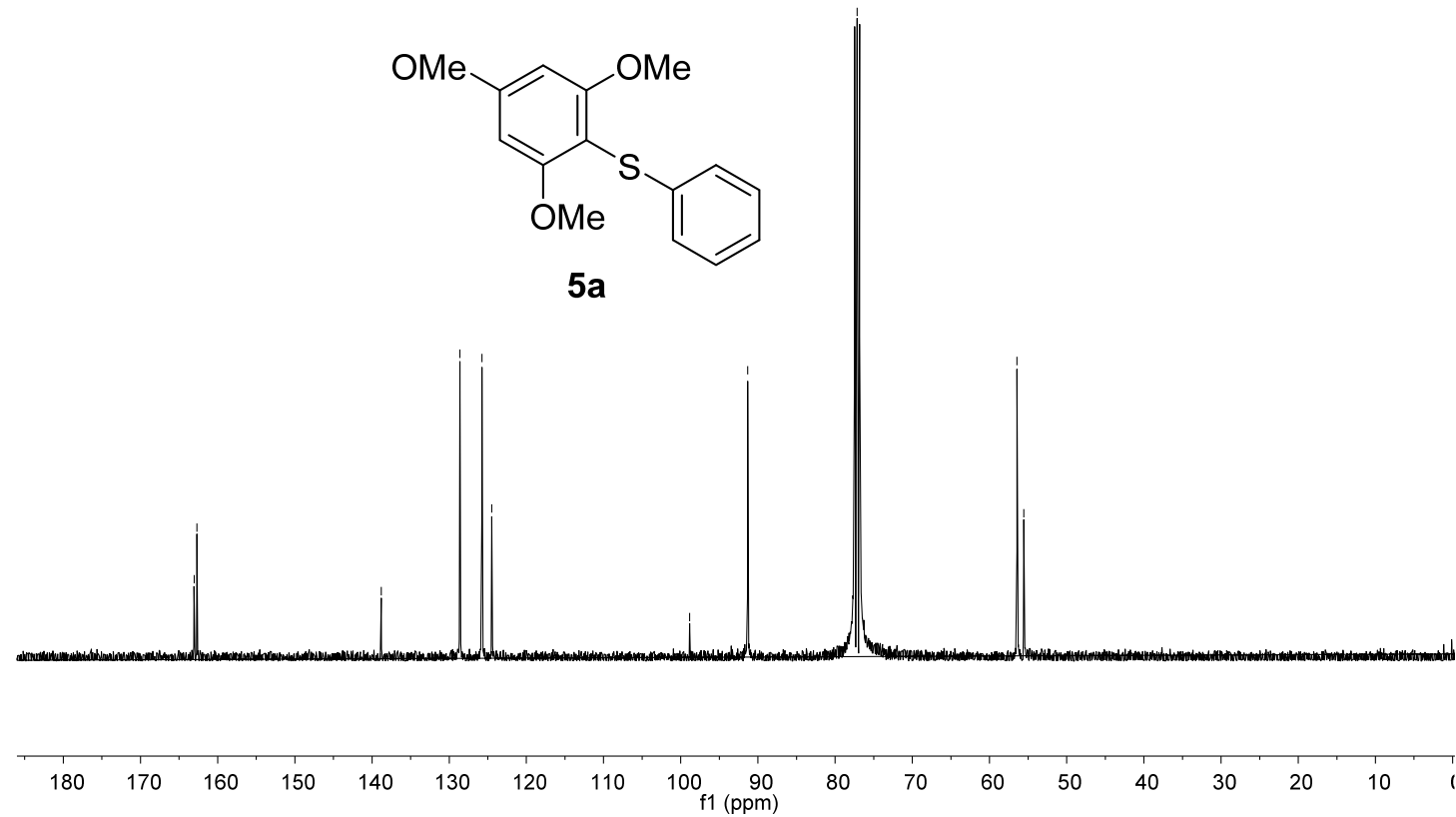

Figure S81

${ }^{1} \mathrm{H}$ and ${ }^{13} \mathrm{C}$ NMR Spectrum of Compound $\mathbf{5 b}$

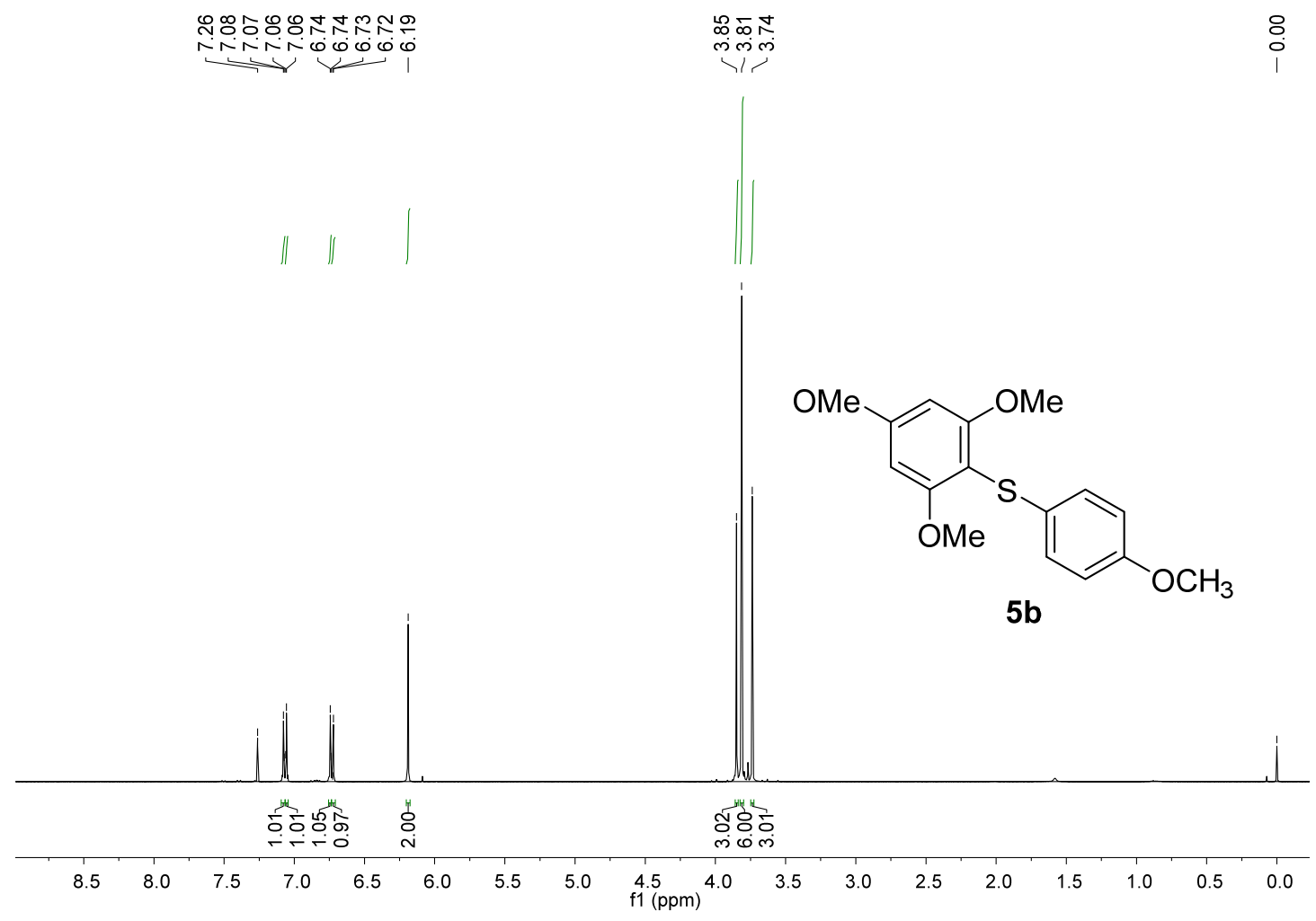

Figure S82 


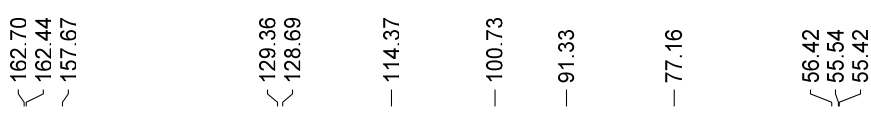

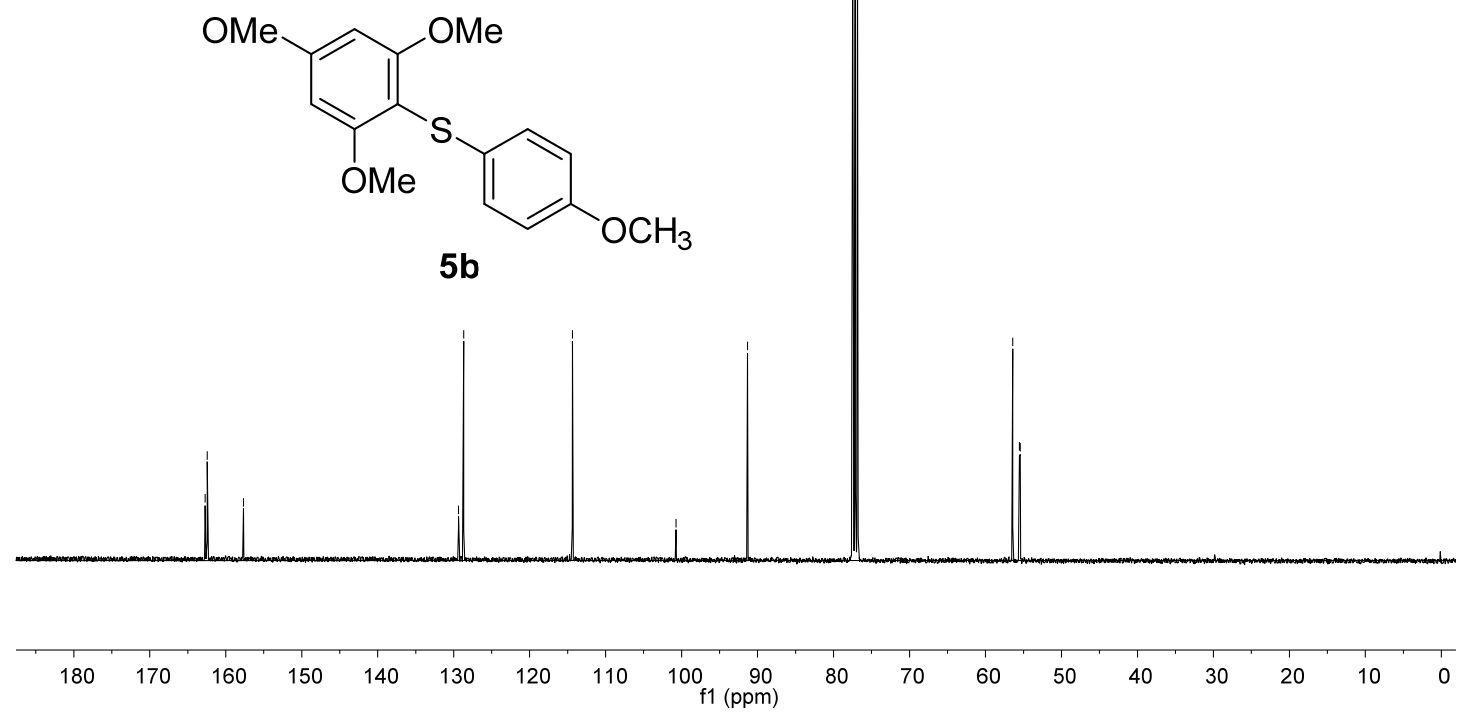

Figure S83

${ }^{1} \mathrm{H}$ and ${ }^{13} \mathrm{C}$ NMR Spectrum of Compound 5c

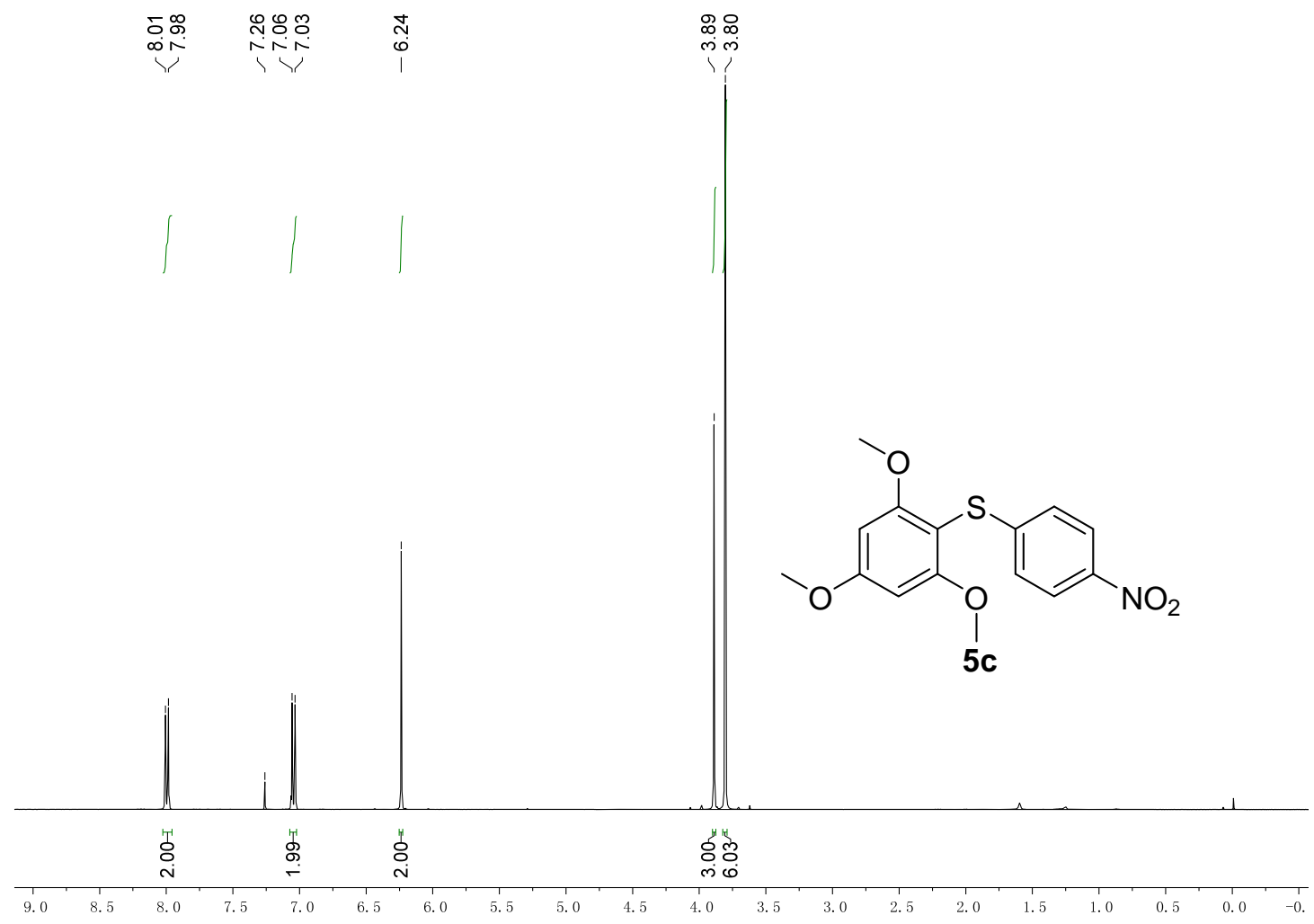

Figure S84 

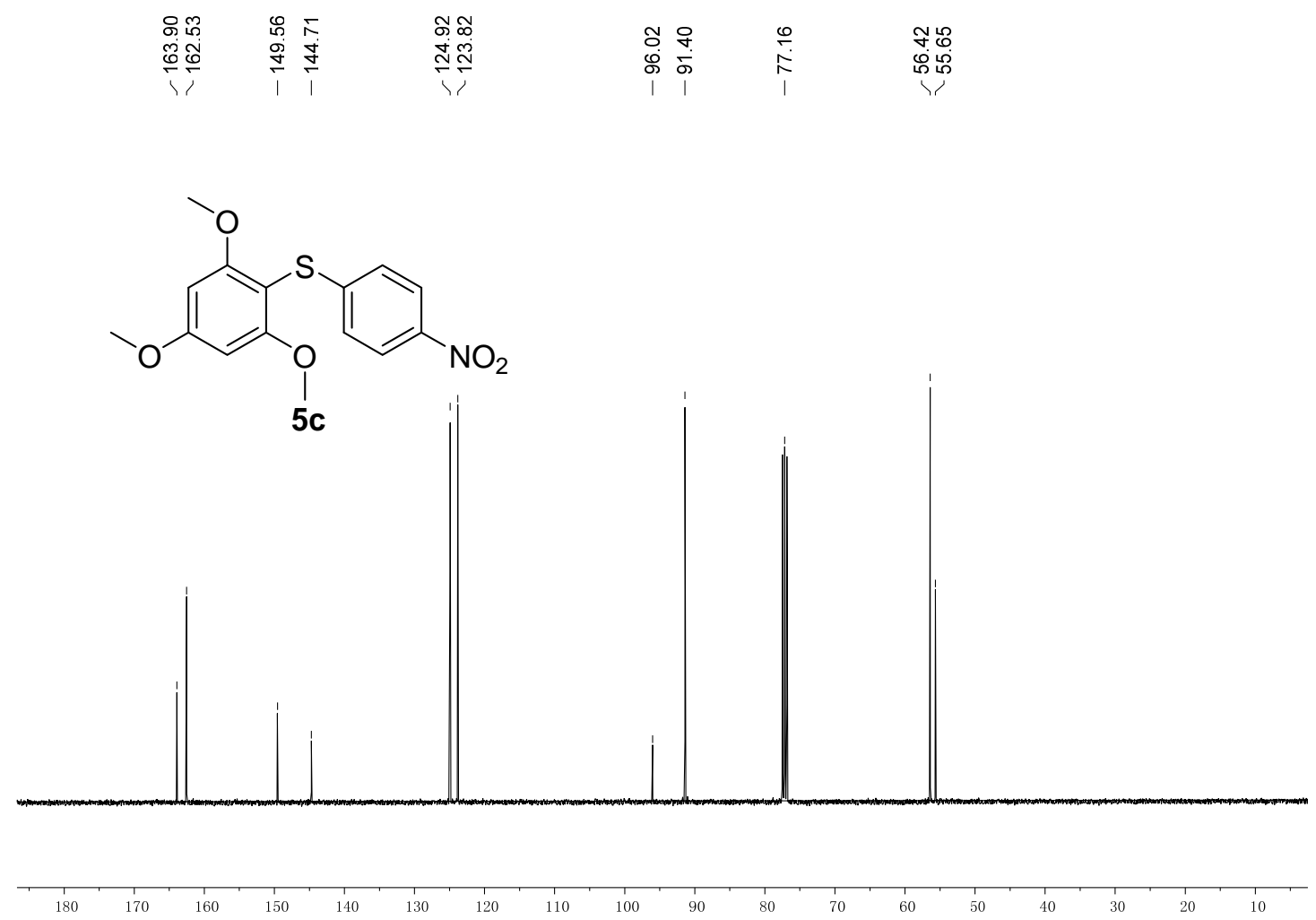

Figure S85

${ }^{1} \mathrm{H}$ and ${ }^{13} \mathrm{C}$ NMR Spectrum of Compound 5d

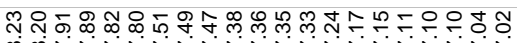

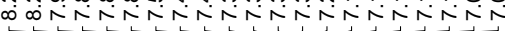
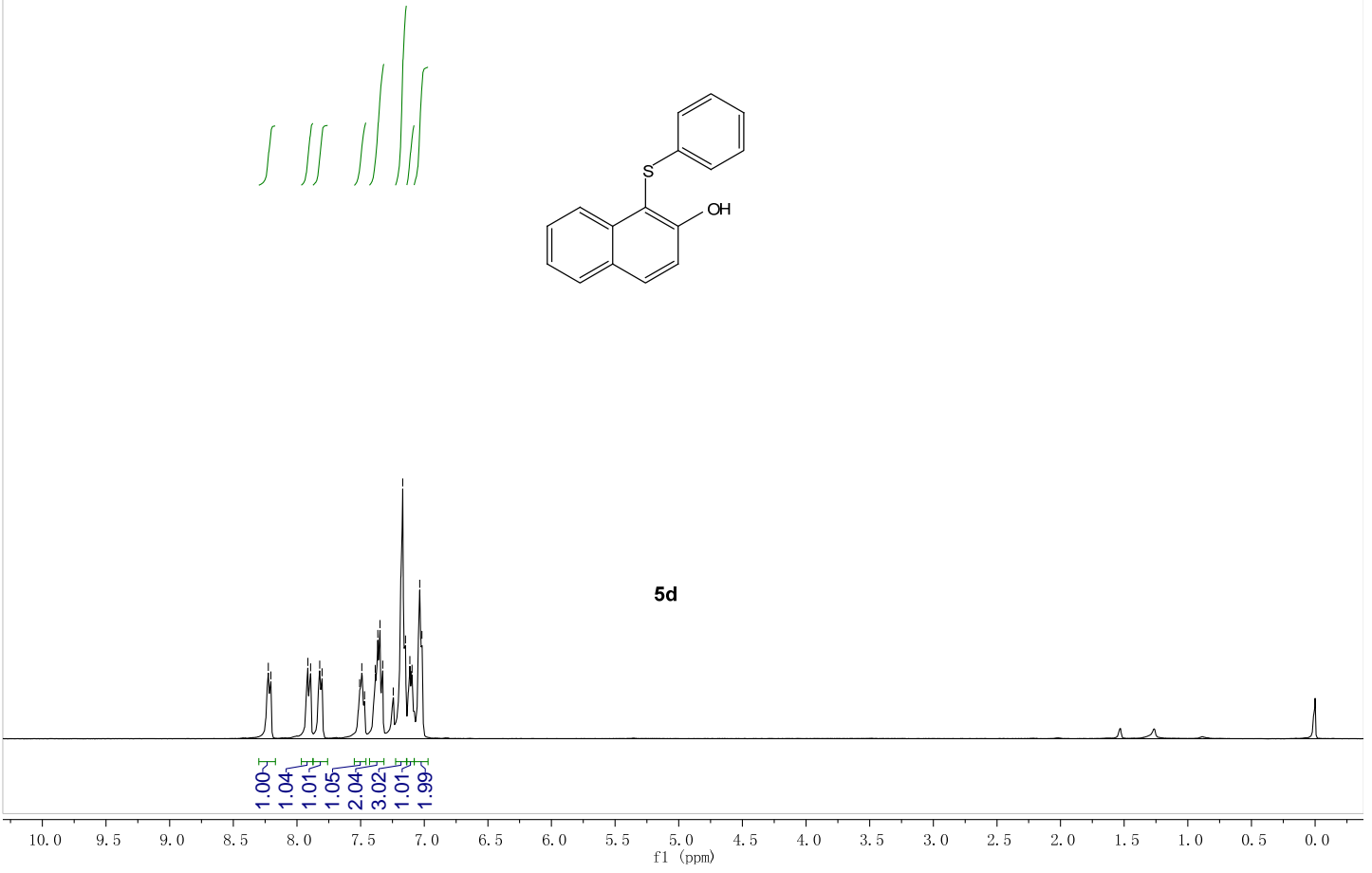

Figure S86 

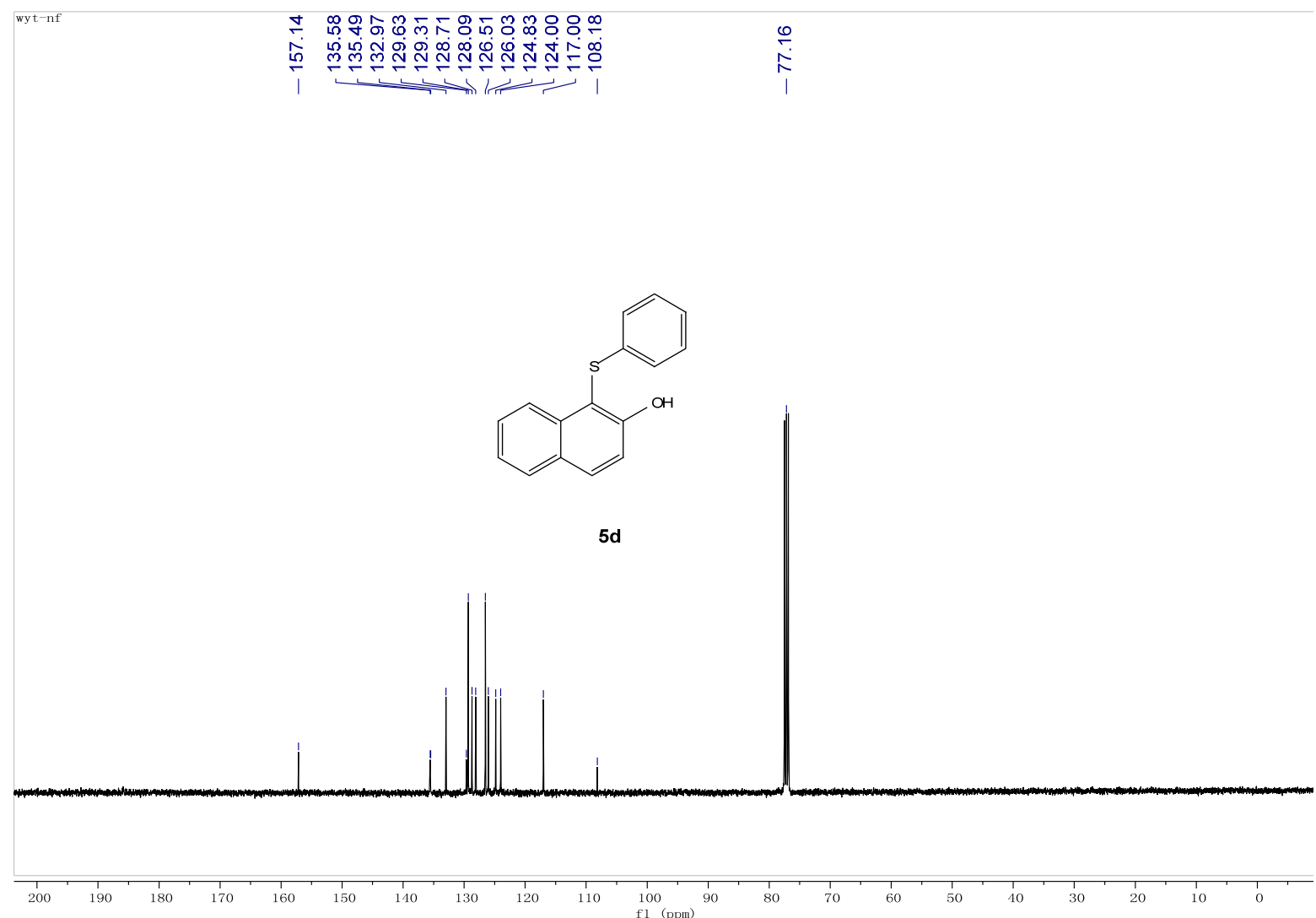

Figure S87

${ }^{1} \mathrm{H}$ and ${ }^{13} \mathrm{C}$ NMR Spectrum of Compound $\mathbf{6 a}$
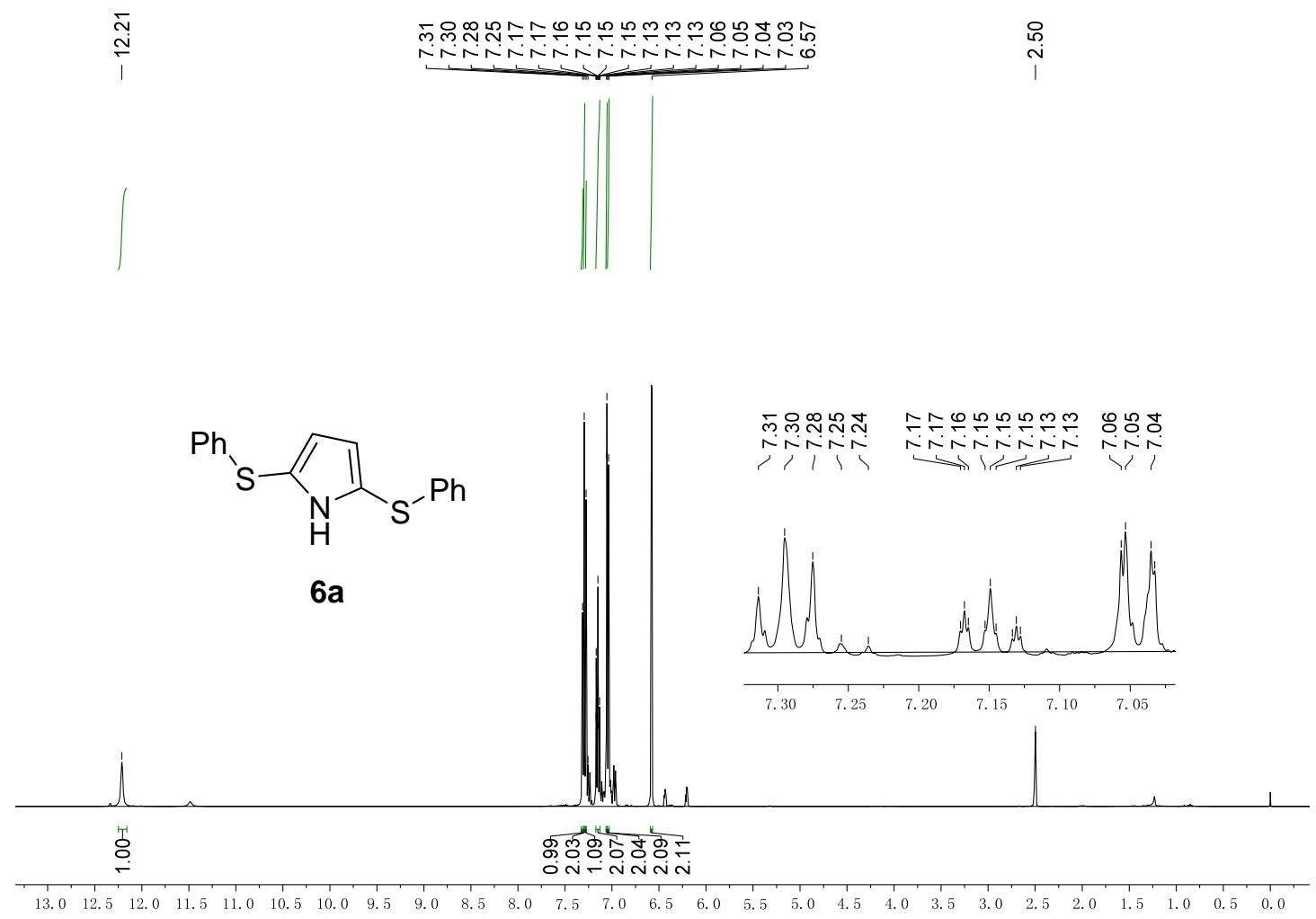

Figure S88 
守

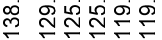
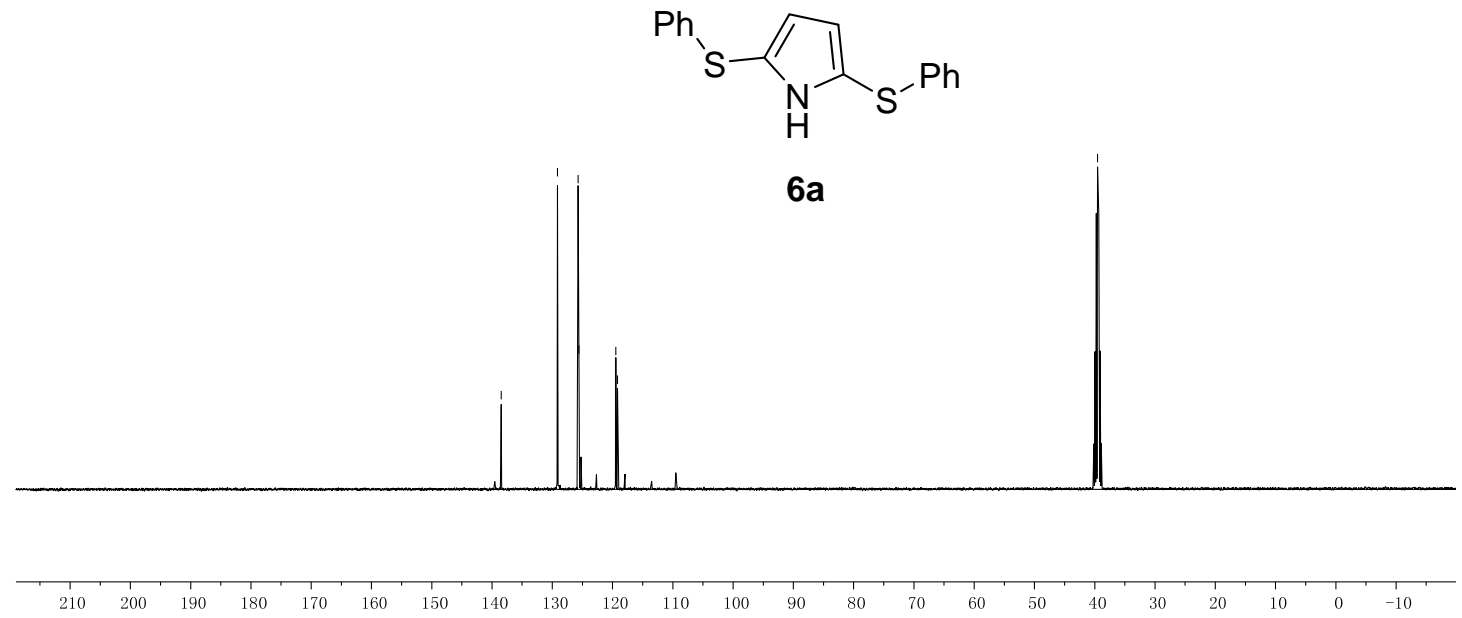

Figure S89

${ }^{1} \mathrm{H}$ and ${ }^{13} \mathrm{C}$ NMR Spectrum of Compound $\mathbf{6 b}$

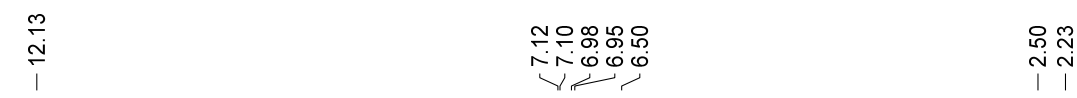

$\int \|$

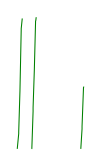

i ก

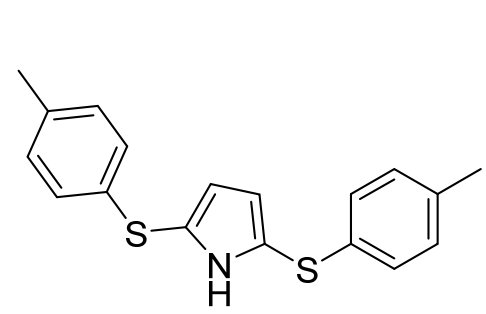

$\stackrel{ }{\stackrel{1}{1}}$

$\begin{array}{ll}\infty & \mathscr{\Omega} \\ 0 & 0 \\ 0 & 0 \\ 1 & 1\end{array}$

$6 b$

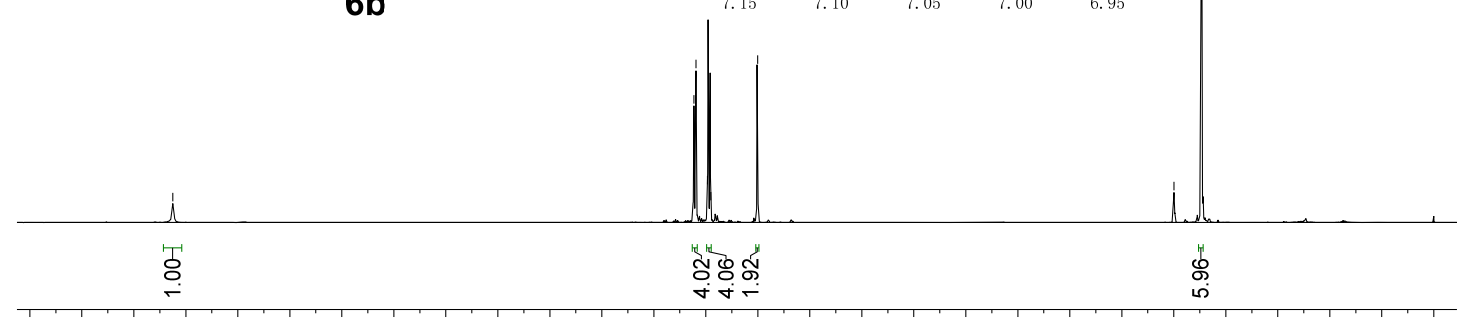

$\begin{array}{llllllllllllllllllllllllllllllll}13.5 & 13.0 & 12.5 & 12.0 & 11.5 & 11.0 & 10.5 & 10.0 & 9.5 & 9.0 & 8.5 & 8.0 & 7.5 & 7.0 & 6.5 & 6.0 & 5.5 & 5.0 & 4.5 & 4.0 & 3.5 & 3.0 & 2.5 & 2.0 & 1.5 & 1.0 & 0.5 & 0.0\end{array}$

Figure S90 


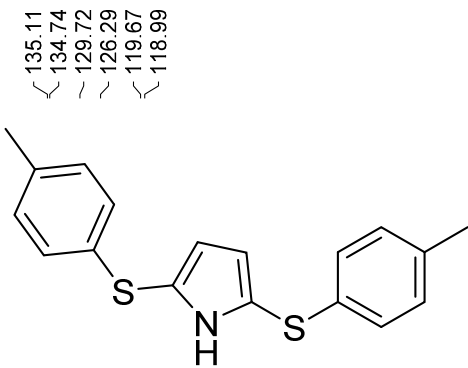

$6 b$
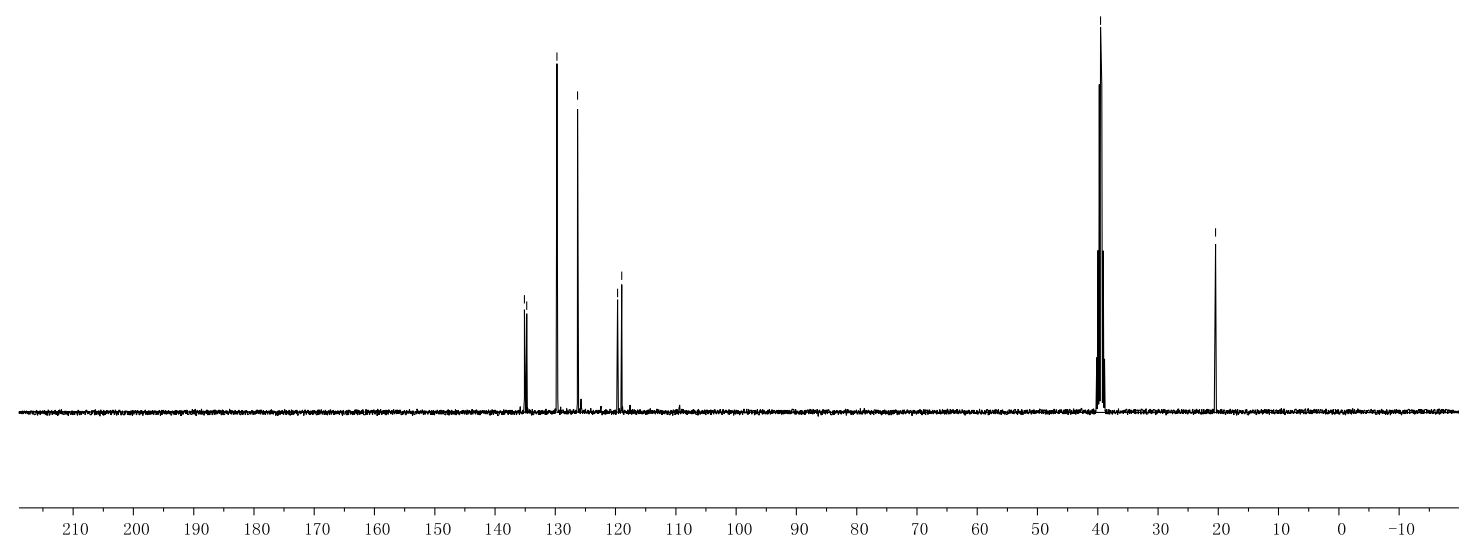

Figure S91

${ }^{1} \mathrm{H}$ and ${ }^{13} \mathrm{C}$ NMR Spectrum of Compound $\mathbf{6 c}$

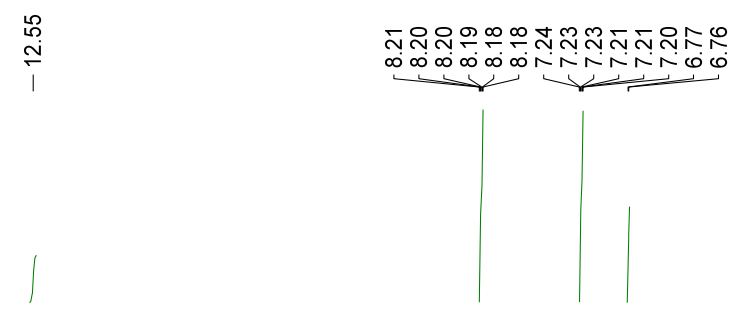

$\stackrel{\circ}{\circ}$
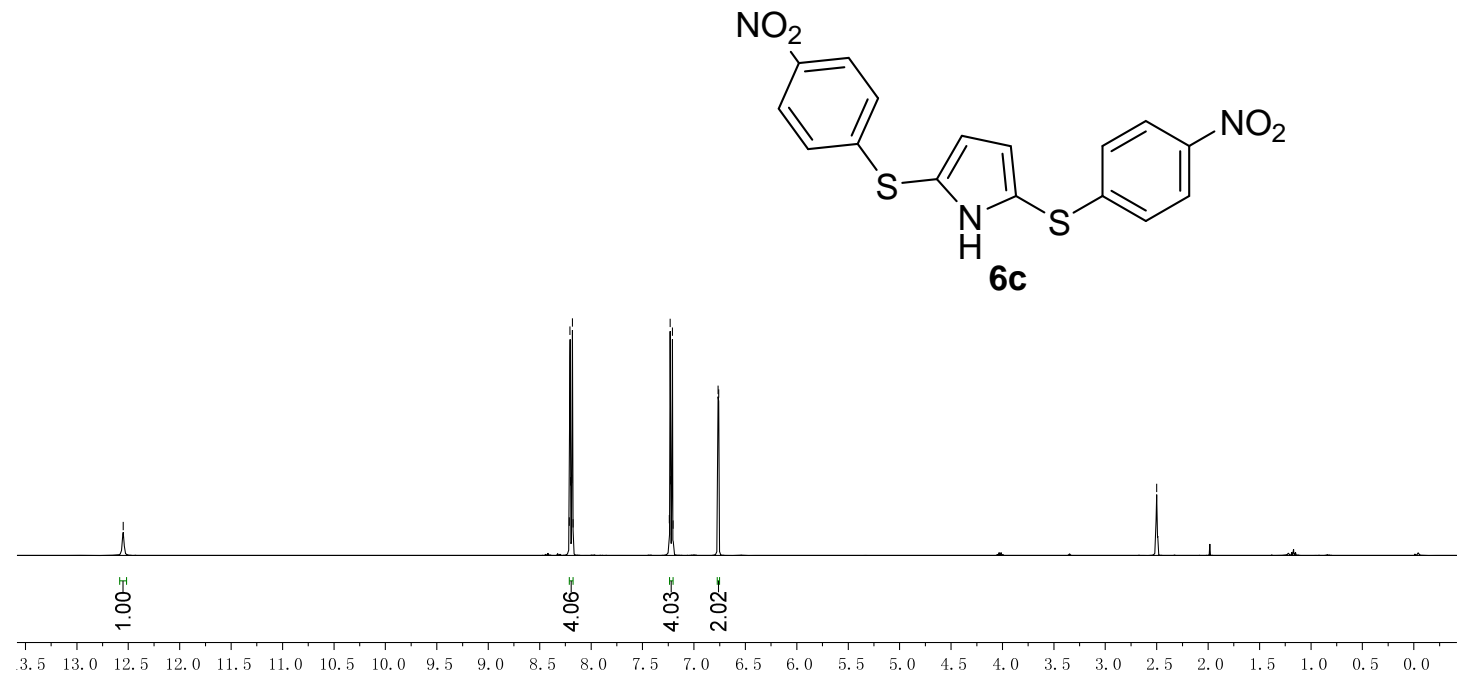

Figure S92 
$\mathrm{NO}_{2}$
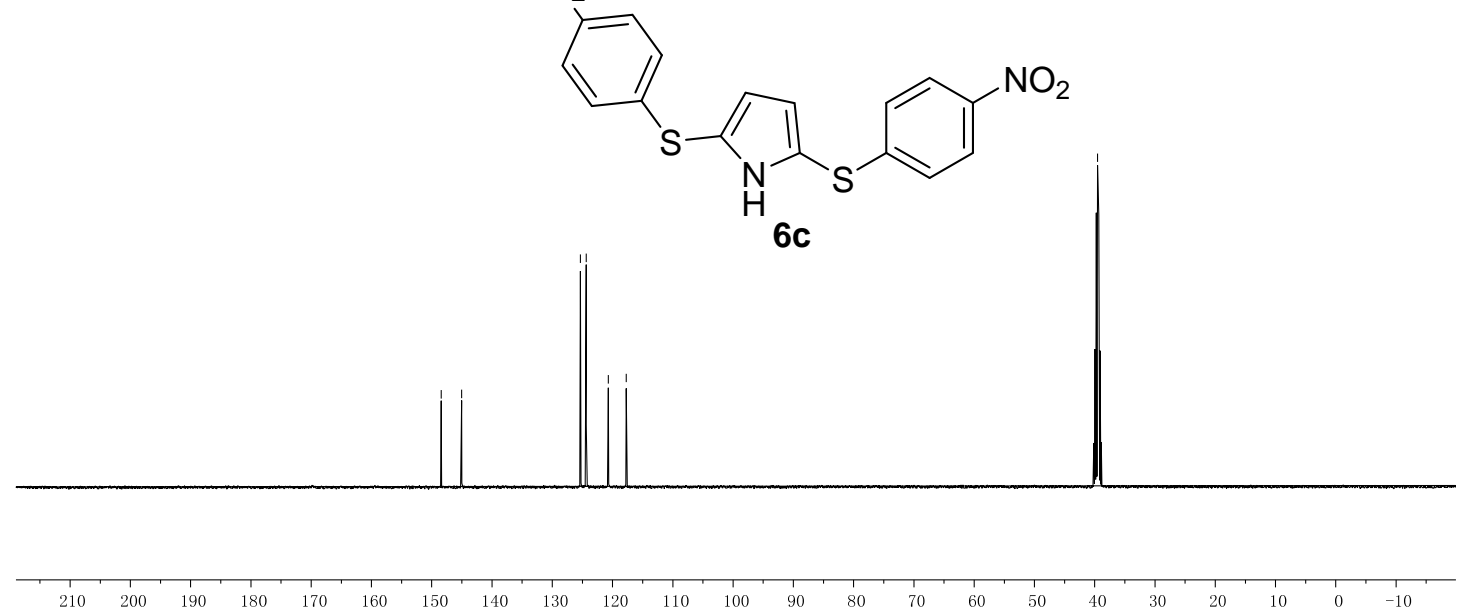

Figure S93

${ }^{1} \mathrm{H}$ and ${ }^{13} \mathrm{C}$ NMR Spectrum of Compound $\mathbf{6 d}$

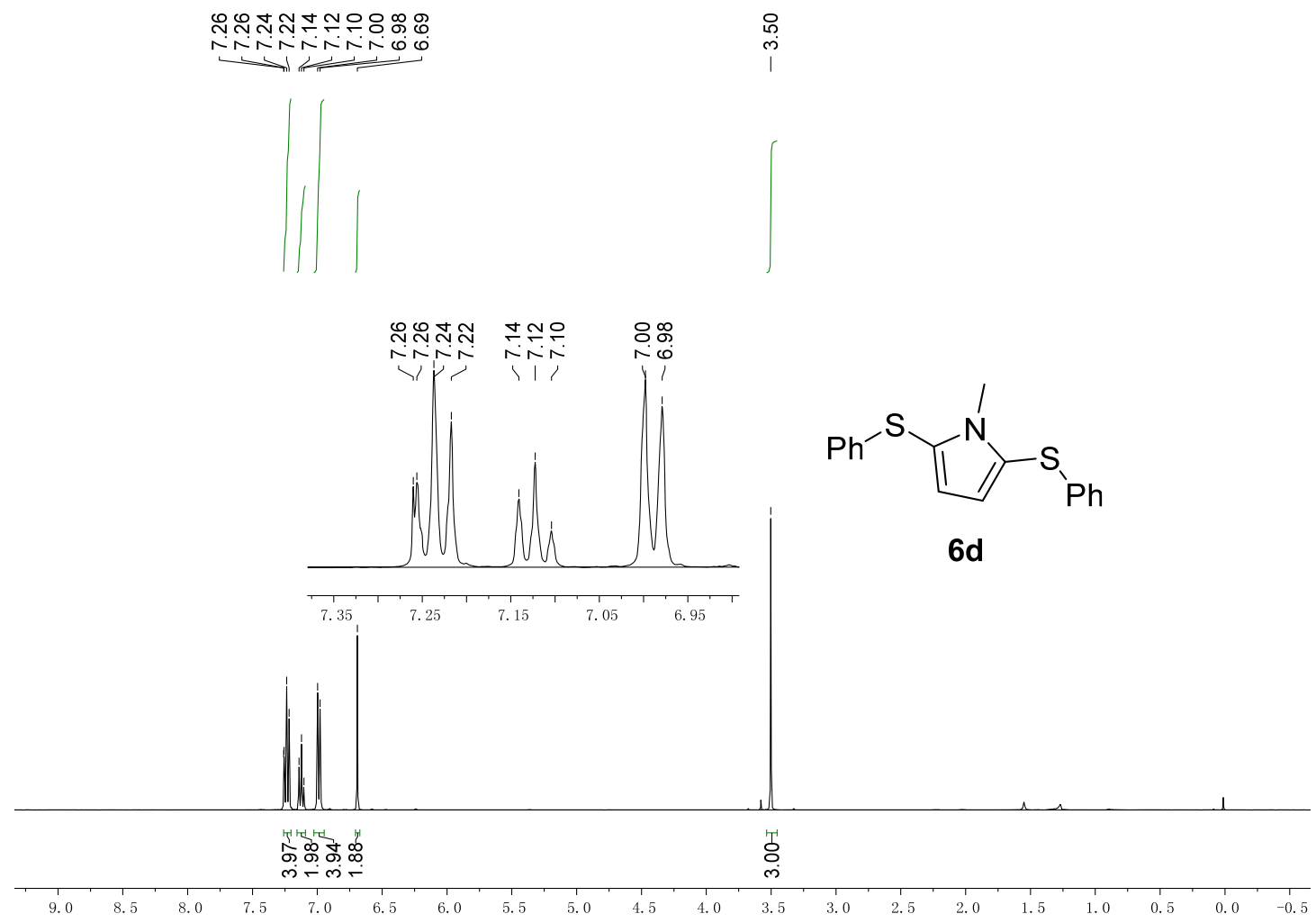

Figure S94 


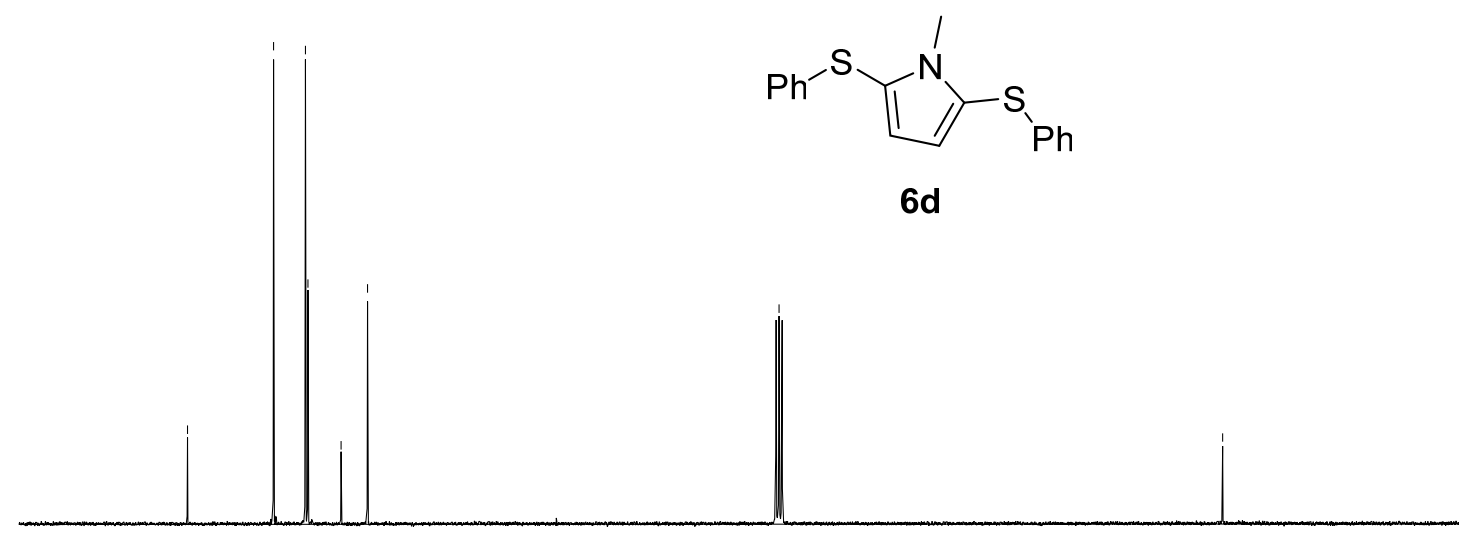

$\begin{array}{llllllllllllllllllllllllllllllllllllllll}555 & 150 & 145 & 140 & 135 & 130 & 125 & 120 & 115 & 110 & 105 & 100 & 95 & 90 & 85 & 80 & 75 & 70 & 65 & 60 & 55 & 50 & 45 & 40 & 35 & 30 & 25 & 20 & 15 & 10\end{array}$

Figure S95

${ }^{1} \mathrm{H}$ and ${ }^{13} \mathrm{C}$ NMR Spectrum of Compound $\mathbf{6 e}$

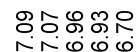

Nंต

1

$\stackrel{\substack{0 \\ i}}{\stackrel{\infty}{N}}$
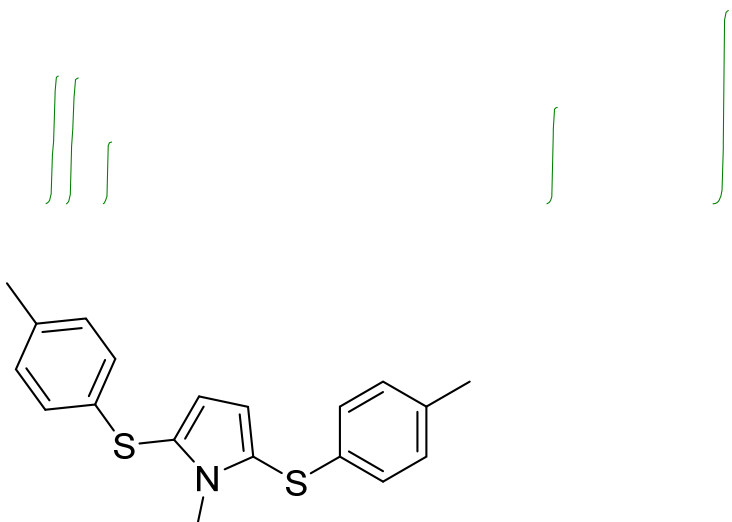

$6 e$

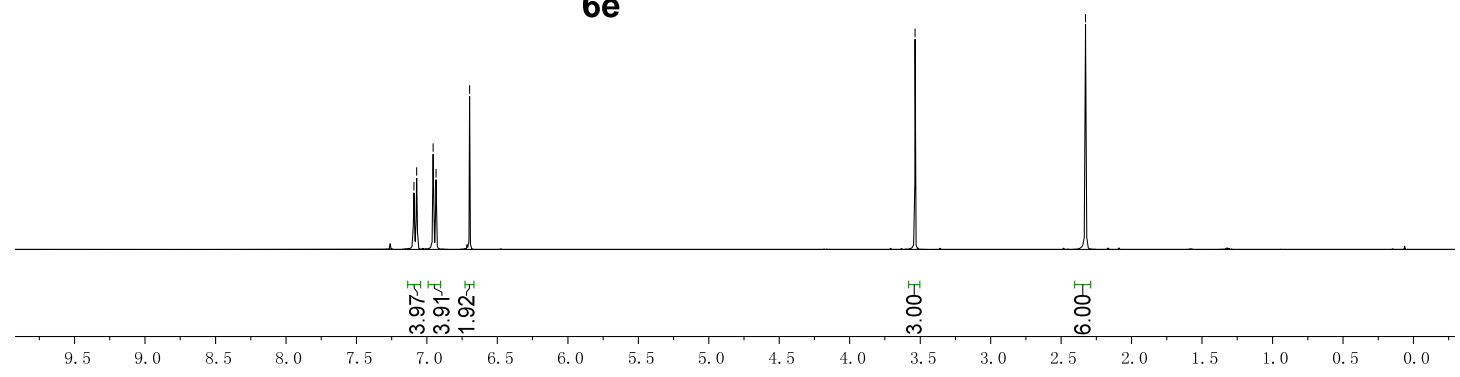

Figure S96 

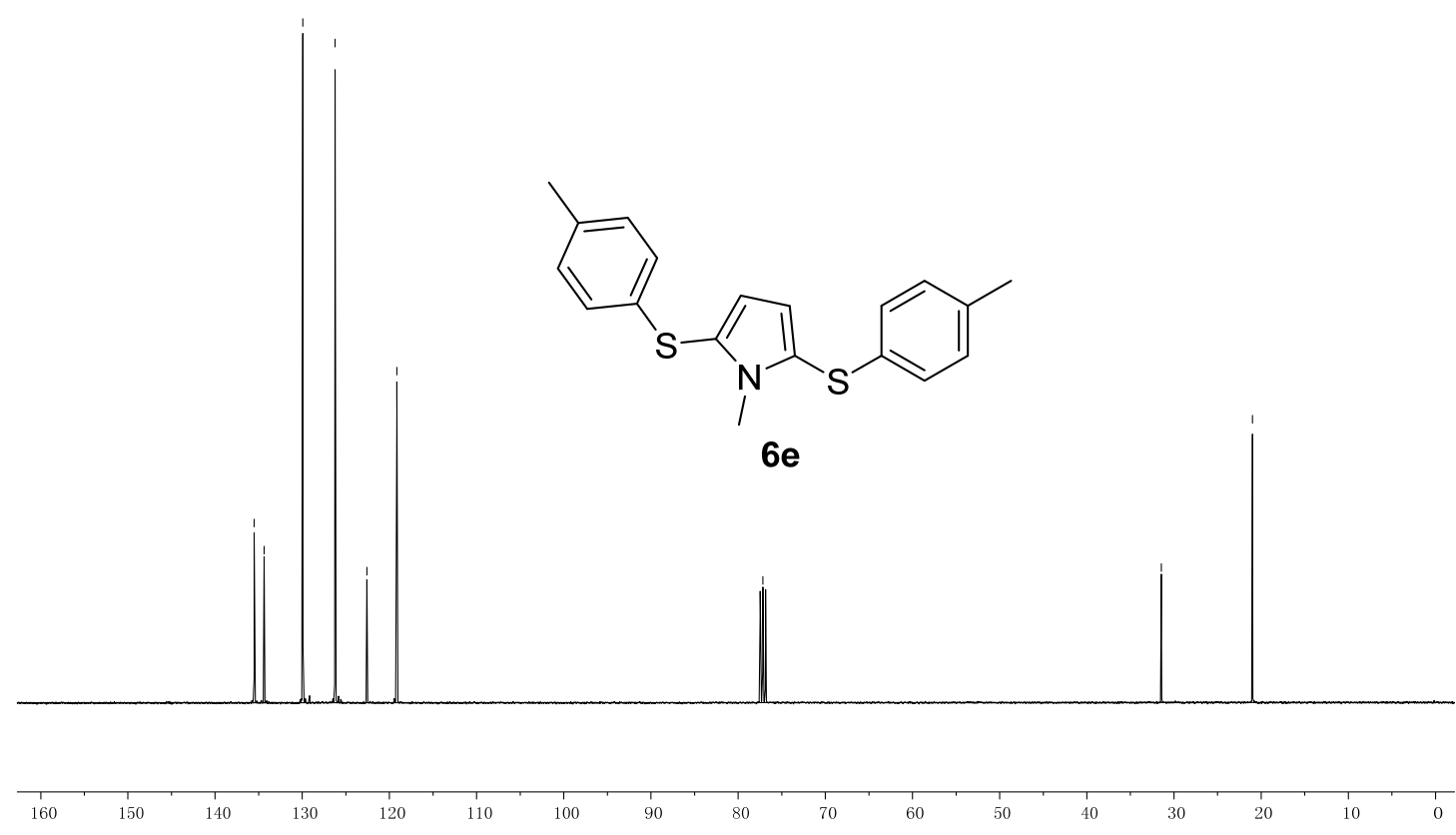

Figure S97

${ }^{1} \mathrm{H}$ and ${ }^{13} \mathrm{C}$ NMR Spectrum of Compound $\mathbf{6 f}$
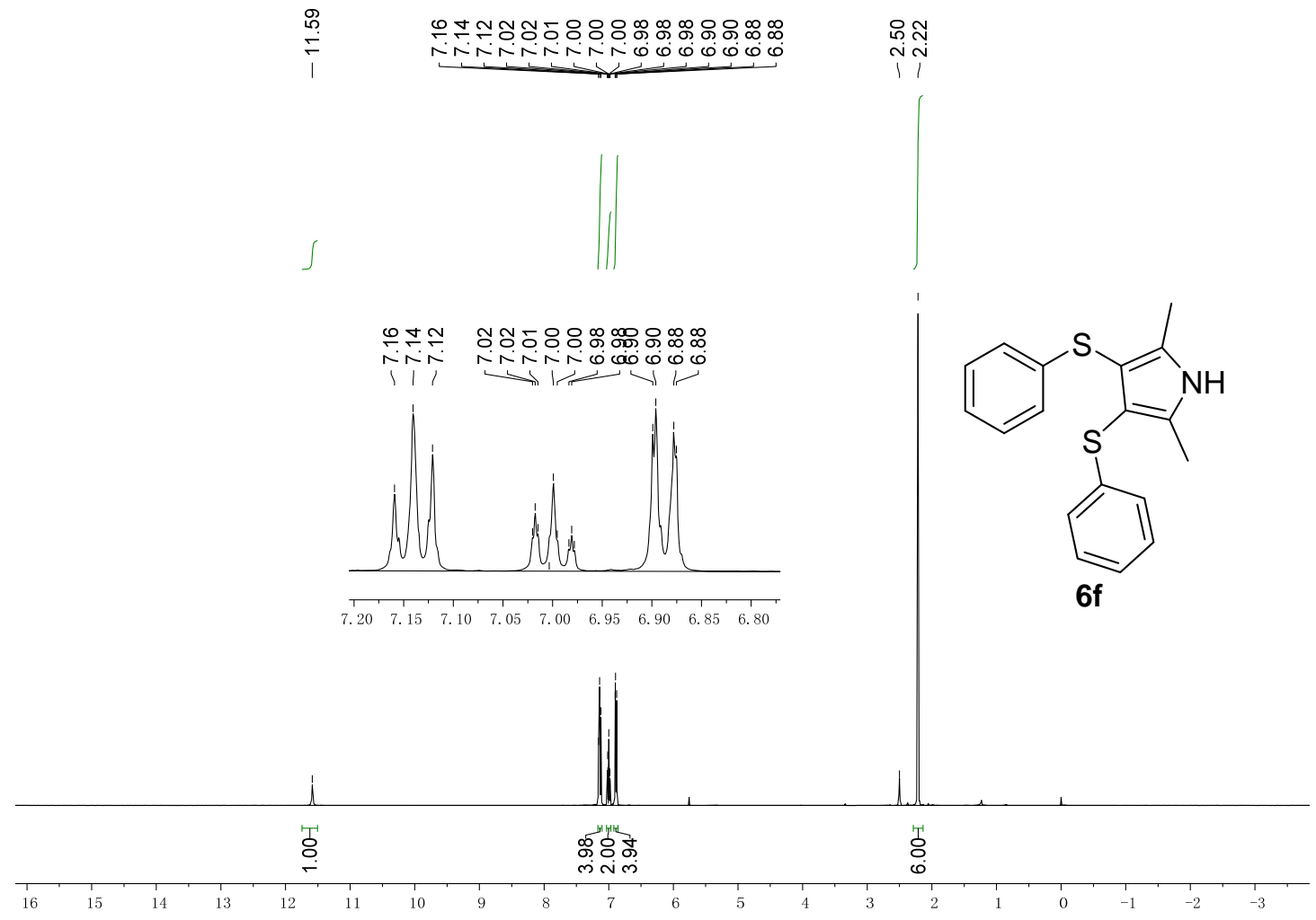

Figure S98 


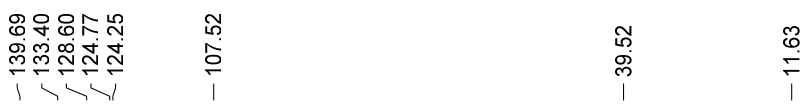<smiles>Cc1[nH]c(C)c(Sc2ccccc2)c1Sc1ccccc1</smiles>

$6 f$
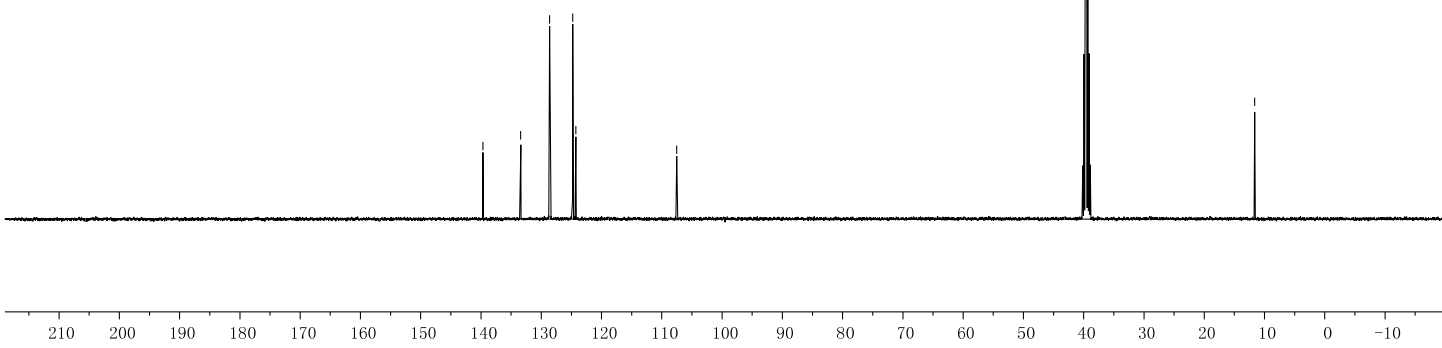

Figure S99

${ }^{1} \mathrm{H}$ and ${ }^{13} \mathrm{C}$ NMR Spectrum of Compound $\mathbf{6 g}$

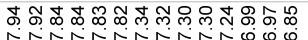

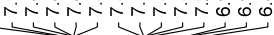
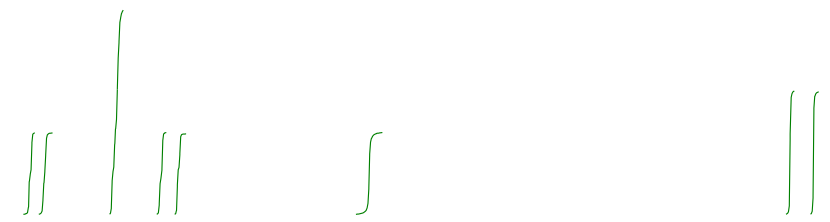<smiles>Cc1ccc(Sc2c(-c3ccccc3)nn(S(=O)(=O)c3ccc(C)cc3)c2N)cc1</smiles>
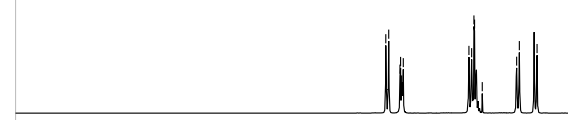

ठำ

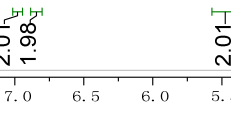

然然

Figure S100 

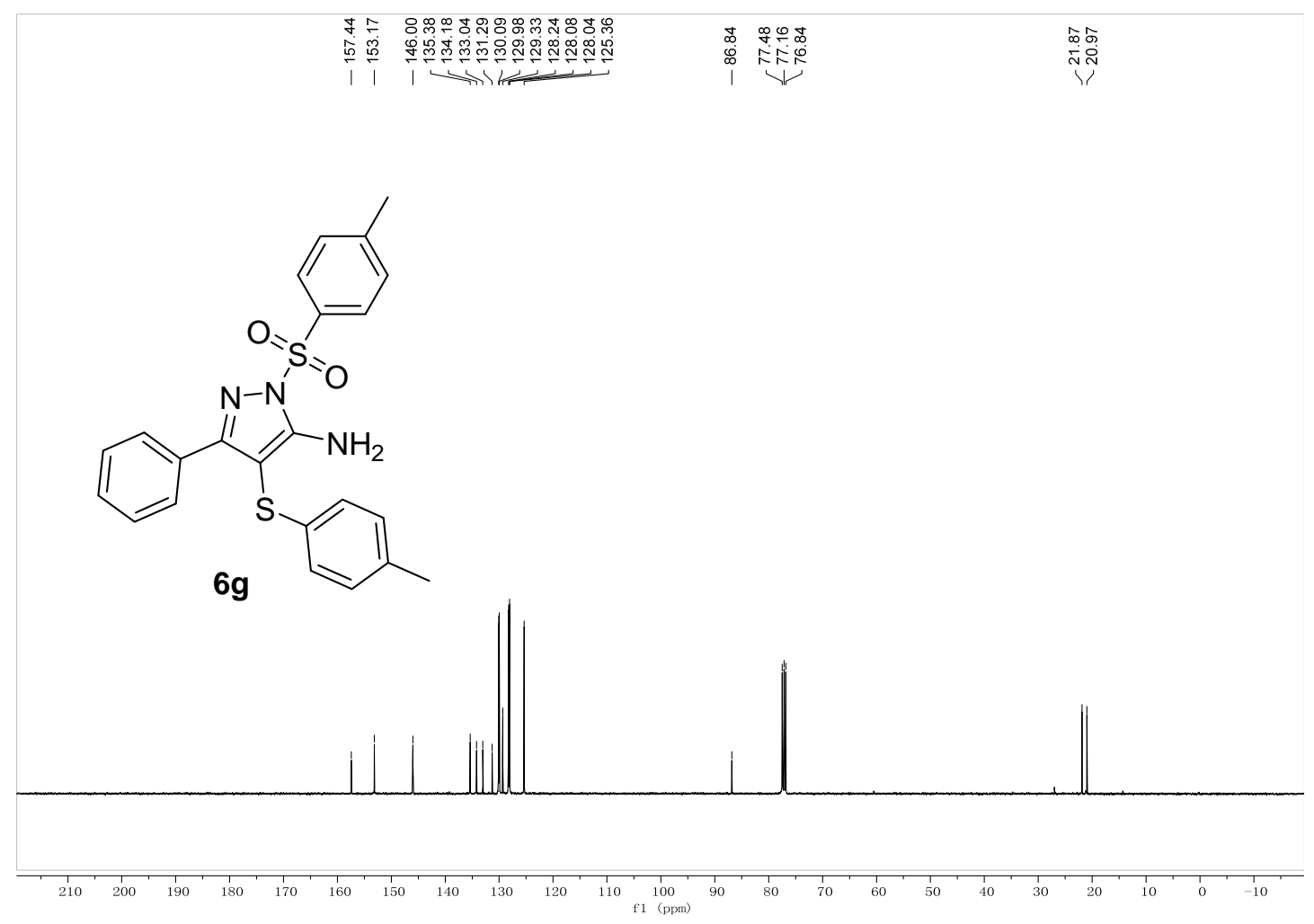

Figure S101

${ }^{1} \mathrm{H}$ and ${ }^{13} \mathrm{C}$ NMR Spectrum of Compound 7a

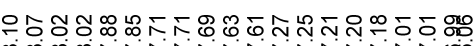

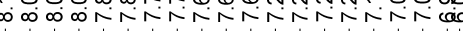

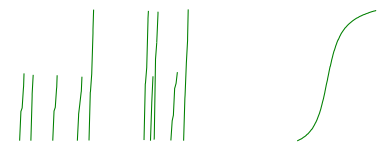<smiles>Cc1ccc(-c2ccc3c(Sc4ccccc4)c(N)ccc3c2)cc1</smiles>

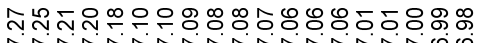

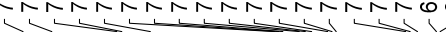

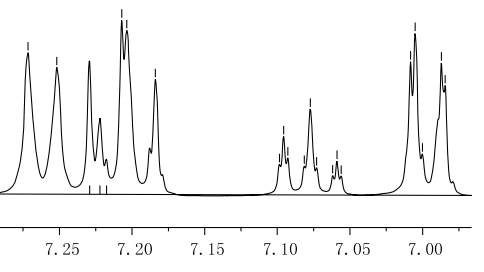

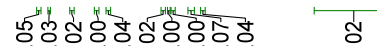
\َ)

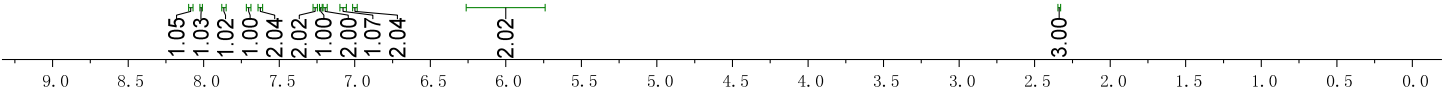

Figure S102 
<smiles>Cc1ccc(-c2ccc3c(Sc4ccccc4)c(N)ccc3c2)cc1</smiles>
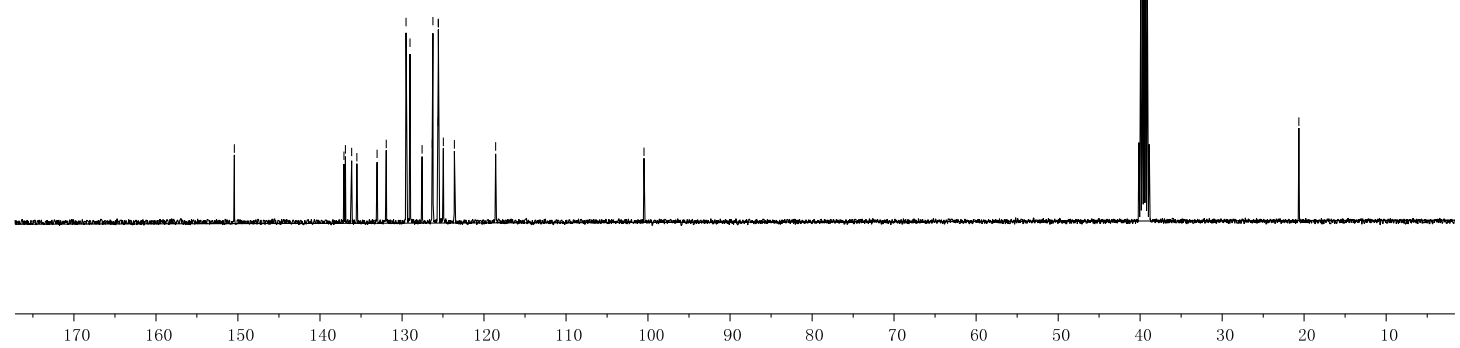

Figure S103

${ }^{1} \mathrm{H}$ and ${ }^{13} \mathrm{C}$ NMR Spectrum of Compound $7 \mathbf{b}$

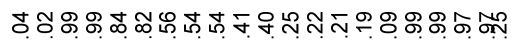
onNNNNNNNNNNR6060
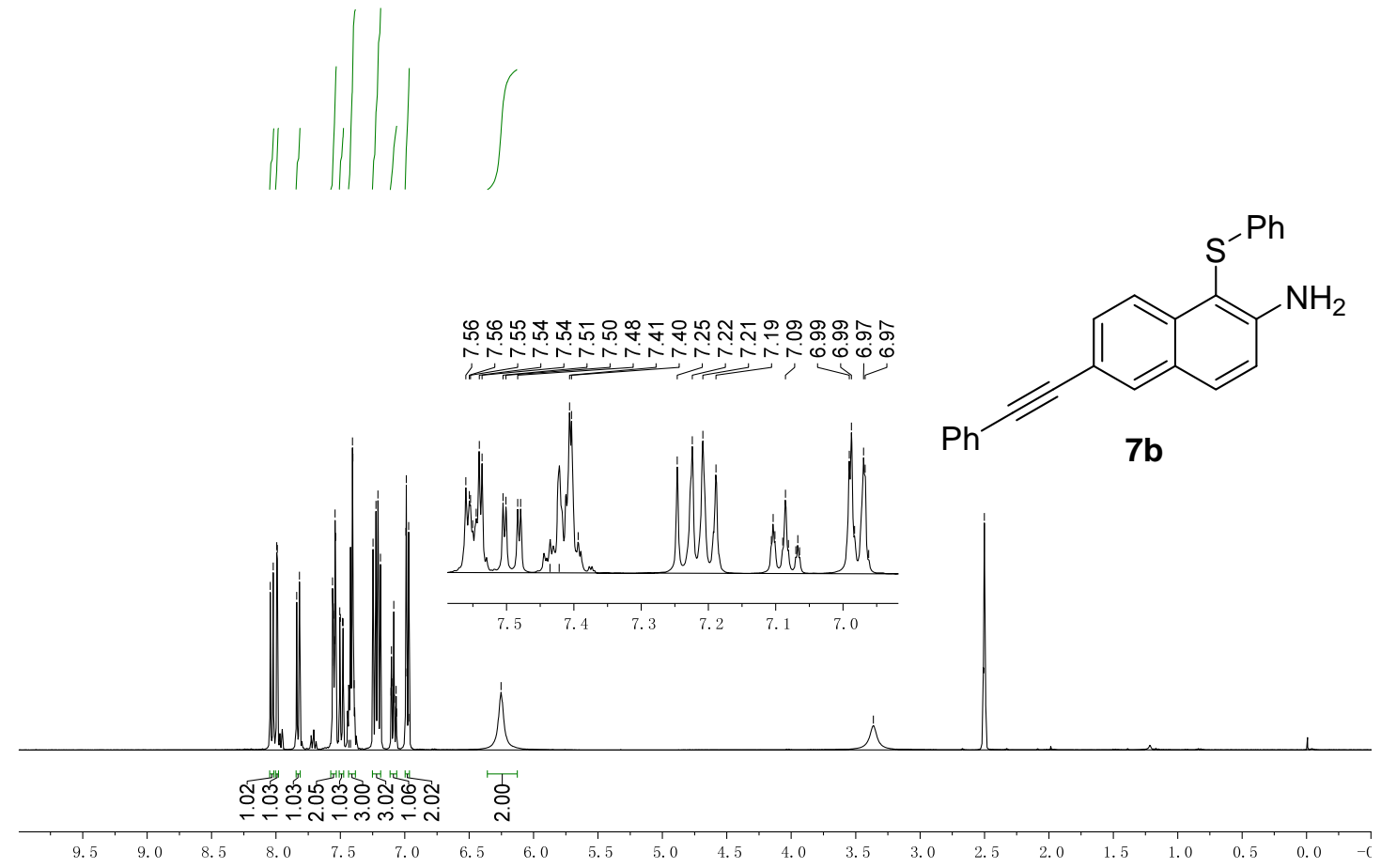

Figure S104 

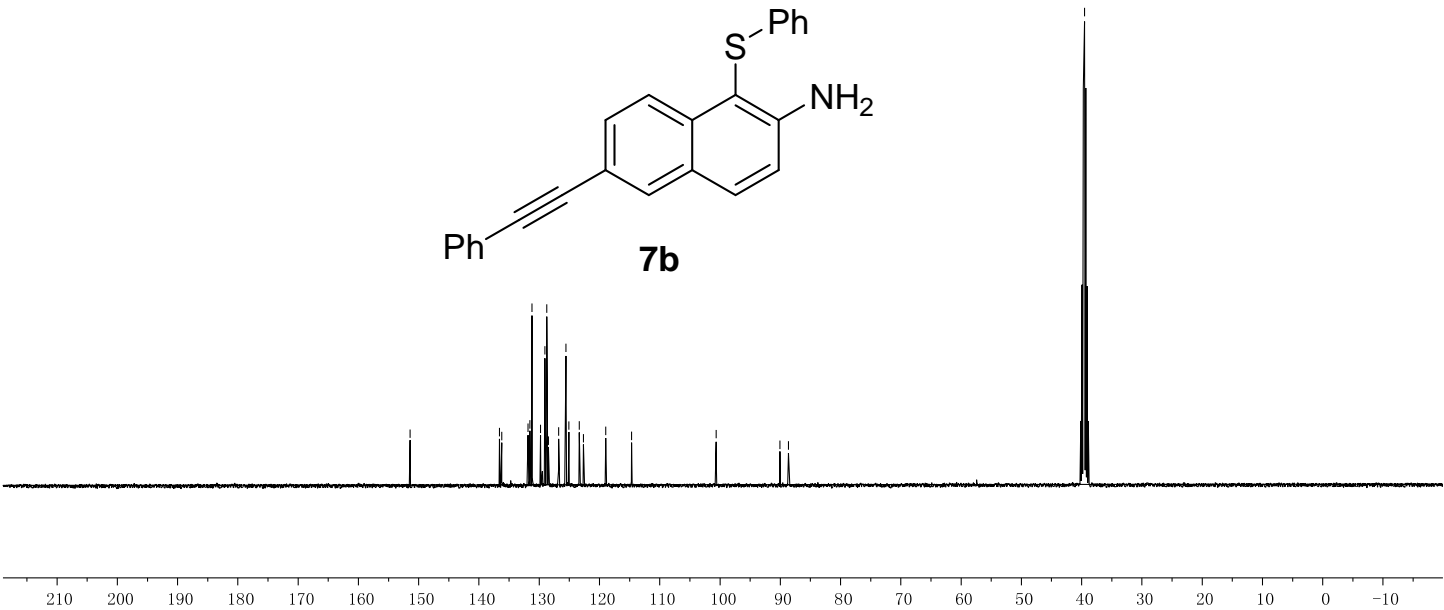

Figure S105

${ }^{1} \mathrm{H}$ and ${ }^{13} \mathrm{C}$ NMR Spectrum of Compound $\mathbf{8 a}$

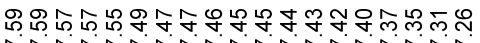

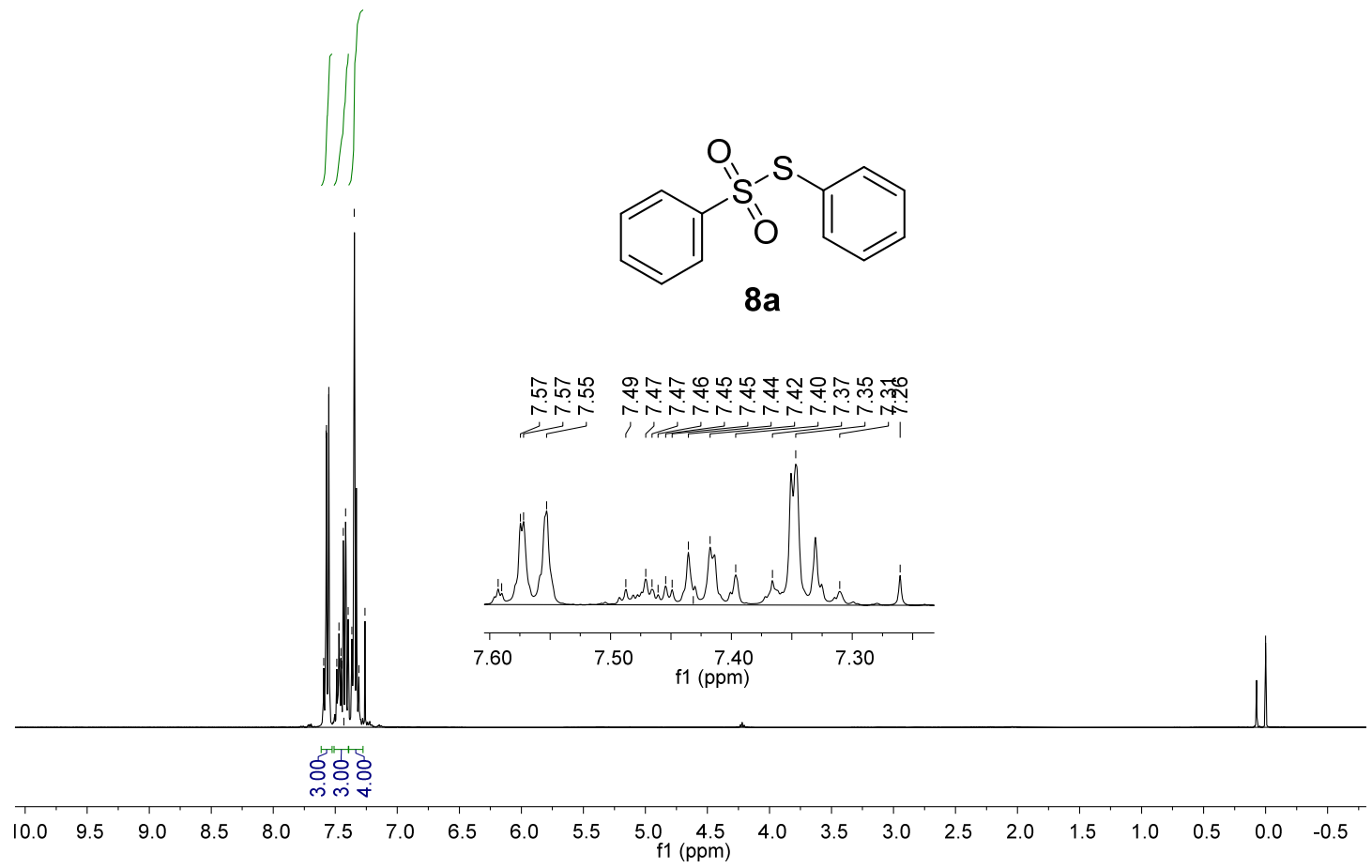

Figure S106 


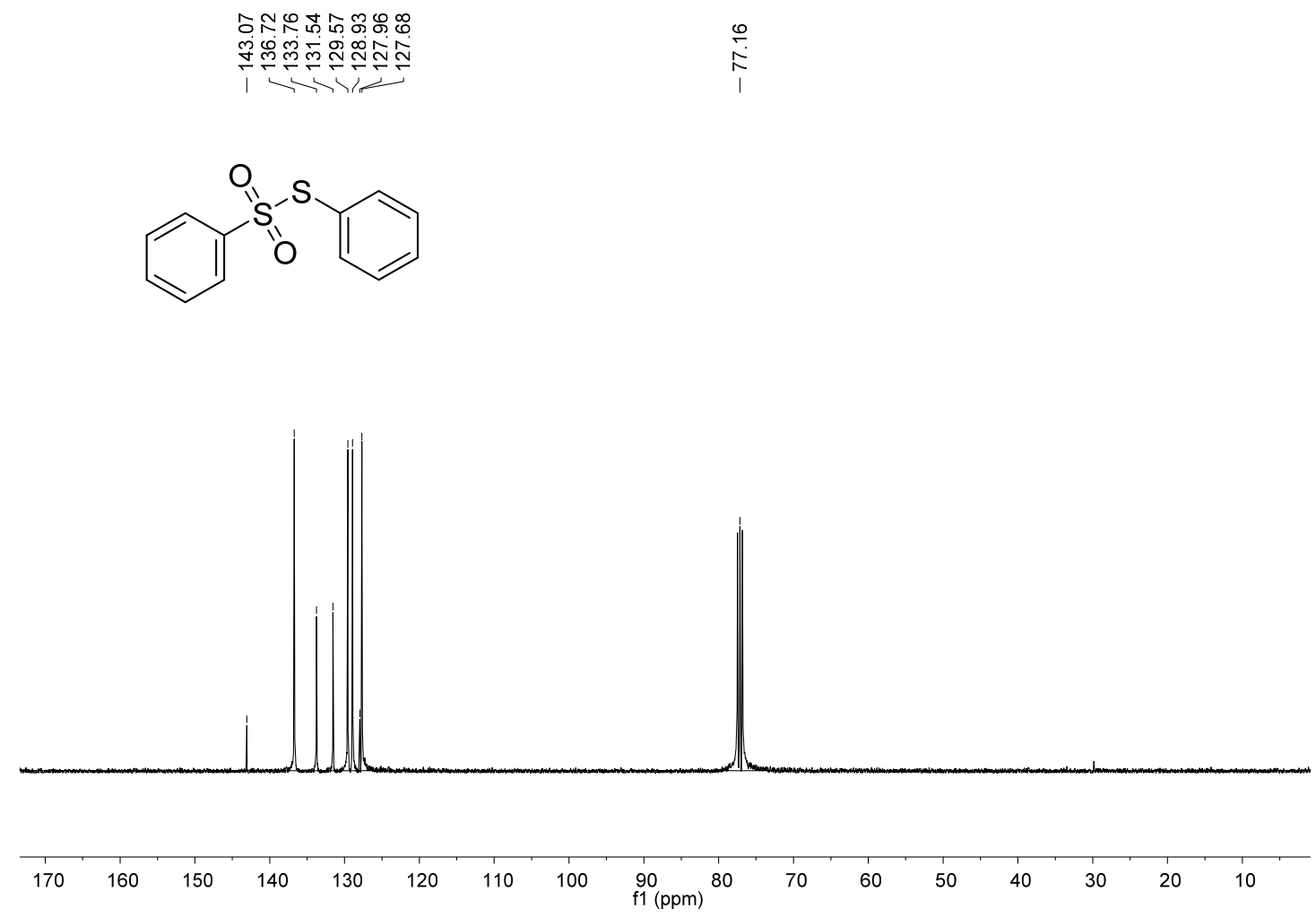

Figure S107 